\title{
Monitoring Accumulated Training and Match Load in Football: A Systematic Review
}

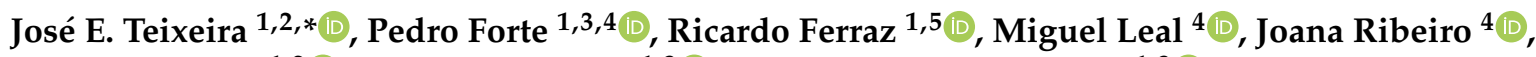 \\ António J. Silva ${ }^{1,2}\left(\mathbb{D}\right.$, Tiago M. Barbosa ${ }^{1,3}\left(\mathbb{D}\right.$ and António M. Monteiro ${ }^{1,3}$ (D) \\ 1 Research Centre in Sports Sciences, Health and Human Development, 5001-801 Vila Real, Portugal; \\ pedromiguel.forte@iscedouro.pt (P.F.); rmpf@ubi.pt (R.F.); ajsilva@utad.pt (A.J.S.); barbosa@ipb.pt (T.M.B.); \\ mmonteiro@ipb.pt (A.M.M.) \\ 2 Department of Sports, Exercise and Health Sciences, University of Trás-os-Montes e Alto Douro, \\ 5001-801 Vila Real, Portugal \\ 3 Departamento de Ciências do Desporto e Educação Física, Instituto Politécnico de Bragança, \\ 5300-253 Bragança, Portugal \\ 4 Department of Sports, Douro Higher Institute of Educational Sciences, 4560-708 Penafiel, Portugal \\ amnfla@gmail.com (M.L.); joana.ribeiro@iscedouro.pt (J.R.) \\ 5 Department of Sports Sciences, University of Beira Interior, 6201-001 Covilhã, Portugal \\ * Correspondence: jose.eduardo@ipb.pt
}

check for

updates

Citation: Teixeira, J.E.; Forte, P.; Ferraz, R.; Leal, M.; Ribeiro, J.; Silva, A.J.; Barbosa, T.M.; Monteiro, A.M. Monitoring Accumulated Training and Match Load in Football: A Systematic Review. Int. J. Environ. Res. Public Health 2021, 18, 3906. https://doi.org/10.3390/ ijerph18083906

Academic Editor: Paul Tchounwou

Received: 10 March 2021

Accepted: 6 April 2021

Published: 8 April 2021

Publisher's Note: MDPI stays neutral with regard to jurisdictional claims in published maps and institutional affiliations.

Copyright: (c) 2021 by the authors. Licensee MDPI, Basel, Switzerland. This article is an open access article distributed under the terms and conditions of the Creative Commons Attribution (CC BY) license (https:// creativecommons.org/licenses/by/ $4.0 /)$.

\begin{abstract}
Background: Training load monitoring has become a relevant research-practice gap to control training and match demands in team sports. However, there are no systematic reviews about accumulated training and match load in football. (2) Methods: Following the preferred reporting item for systematic reviews and meta-analyses (PRISMA), a systematic search of relevant English-language articles was performed from earliest record to March 2020. The search included descriptors relevant to football, training load, and periodization. (3) Results: The literature search returned 7972 articles $($ WoS $=1204 ;$ Pub-Med $=869$, SCOPUS $=5083$, and SportDiscus $=816)$. After screening, 36 full-text articles met the inclusion criteria and were reviewed. Eleven of the included articles analyzed weekly training load distribution; fourteen, the weekly training load and match load distribution; and eleven were about internal and external load relationships during training. The reviewed articles were based on short-telemetry systems $(n=12)$, global positioning tracking systems $(n=25)$, local position measurement systems $(n=3)$, and multiple-camera systems $(n=3)$. External load measures were quantified with distance and covered distance in different speed zones $(n=27)$, acceleration and deceleration $(n=13)$ thresholds, accelerometer metrics $(n=11)$, metabolic power output $(n=4)$, and ratios/scores $(n=6)$. Additionally, the internal load measures were reported with perceived exertion $(n=16)$; heart-rate-based measures were reported in twelve studies $(n=12)$. (4) Conclusions: The weekly microcycle presented a high loading variation and a limited variation across a competitive season. The magnitude of loading variation seems to be influenced by the type of week, player's starting status, playing positions, age group, training mode and contextual variables. The literature has focused mainly on professional men; future research should be on the youth and female accumulated training/match load monitoring.
\end{abstract}

Keywords: performance; periodization; training control; match demands

\section{Introduction}

Football is a team sport characterized by intermittent efforts, combining high-speeds and intensity with low-intensity periods [1,2]. Knowing about the match physical and physiological demands allows to carry out the training mode [3]. The training process requires a systematic and periodized application to ensure optimal adaptations to physiological responses and biochemical stresses [4,5]. Researchers and practitioners aim to promote favorable performance outcomes and an adequate recovery for match demands [5]. 
Football training programs may improve aerobic and anaerobic fitness; these adaptations should be monitored and controlled periodically [6]. The training load has been defined as an input variable for training outcomes, allowing to control training session demands in real time and after each training sessions [7]. The training load can be split up into external (physical) and internal (physiological) load, providing insights about dose-response [6,7]. The external load is defined as the performed work during training sessions or competition, regardless of the internal characteristics. The external load can be monitored by global positioning systems (GPS) tracking systems [8], micro-electromechanical systems (MEMS) [9], local position measurement (LPM), and computerized-video systems [10]. Commonly, external load measures are power output, distances, speeds, accelerations/decelerations, time-motion analysis, and neuromuscular function $[5,11]$. The internal load refers to physiological and psychological stress and is possible to assess by objective and subjective instruments [5,7]. The most commonly used objective measures are the physiological, such as heart rate, lactate, or oxygen consumption; and training impulse (TRIMP). Moreover, subjective measures usually include ratings of perceived exertion, wellness questionnaires, and psychological inventories [5,12].

The training effects depend on physiological stimulus by intensity, duration, frequency, and recovery periods $[6,13]$. The external load provides training quality, quantity, and organization; quantifying their components allows an overview of training prescription $[4,14]$. The physiological adaptations have been well documented [1,2]. However, there is no unique physiological marker that can be used to assess the fitness-fatigue binomial to predict performance [12]. Combining internal and external load data can be used as an approach to overcome the conceptual barrier concerning the fitness-fatigue binomial [15]. However, there is no consensus of an effectiveness monitoring system in professional football [16]. The training load quantification in team sports is often mentioned as a great challenge. This may be due to the difficulty of accurately assessing the skilled performance and cognitive load that influences decision-making [17]. Furthermore, the diversity of monitoring tools appears to have created confusion in dose-response considerations. Indeed, turning these data into relevant information has become a significant challenge to coaches and sport scientists [18].

Currently, a growing number of articles have been published on training load. Recent reviews and meta-analysis focused on team sports aimed to evaluate the association between loading and performance [19,20], intensity [21], training outcomes [22], acute/residual fatigue [23,24], and injury, illness, and soreness [24]. The use of microtechnology to collect and interpret training load has been largely revised in team sports $[25,26]$ and particularly in professional football [27]. Youth football has also been revised with the objective of match running performance [28] and injury incidence [29]. The match running performance has been widely described considering playing position, formation, and opposition standard [30-33]. However, there have been no previously published systematic reviews and/or meta-analyses about monitoring accumulated training and match load [24]. The match-play represents the greatest physiological stimulus and represents the primary performance outcome [32]. Nonetheless, nearly $80 \%$ of the weekly training load results from the training sessions whereas about $20 \%$ is from the match-play $[1,34]$. Understanding the cumulative effect of training is essential to guide the individual athlete's performance $[3,5]$.

Cumulative effect is a primary factor for the long-term training process and athletic preparation [35]. Training load monitoring plays an important role in training periodization and evaluating cumulative effects variation is essential to an effective training planning according to the individualization principle [36]. Previous research has focused on match load [37] or quantifying training load in specific training moments and highly controlled situations using constrained tasks [38,39]. Monitoring gross and temporal demands during training sessions may help to improve ecological validity. Even more, it may allow to supply an accurate understanding about the inclusion of training load measures in training practices and match management $[15,35]$. However, there is a lack of consensus on the most 
effective strategies and training load metrics to measure accumulative training and match demands [16]. Additionally, the different methodologies could lead to outcome differences and bias in the loading analysis [40]. Understanding the seasonal training/match load variations and the relationships between measures would appear important to define the most appropriate monitoring strategy. Therefore, the purpose of this systematic review was three-fold; (1) to analyze intra and inter-individual accumulative training load distribution within a week (microcycle), weeks (mesocycle), and/or season phases; (2) to analyze the intra and inter-individual accumulative training and match load distribution within a week (microcycle), weeks (mesocycle), and/or season phases; and (3) to analyze relationships between internal and external load measures in the accumulative training load quantification.

\section{Materials and Methods}

The present systematic review protocol was registered at the International Platform of Registered Systematic Review and Meta-Analysis Protocols with the number 202080095 and doi:10.37766/inplasy2020.8.0095.

\subsection{Literature Search Strategy}

The preferred reporting items for systematic reviews and meta-analyses (PRISMA) guidelines and the population-intervention-comparators-outcomes (PICOS) design were followed to conduct this systematic review [41,42]. The literature search was based on four databases: PubMed/Medline, Web of Science (WoS, including all Web of Science Core Collection: Citation Indexes), SCOPUS, and SportsDiscus. The eligibility criteria were assured by a PICOS approach and the following search strategy was defined: (1) population: adult and youth football players (participants aged < 13 years); (2) intervention: quantify and compare external (physical) and internal (physiological) load during at least a 1-week period (microcycle); (3) comparison: periodization structure (microcycle, mesocycle, and/or season phase); (4) outcomes: intra- and inter-individual accumulative load distribution; and (5) study design: experimental and quasi-experimental trials (e.g., randomized controlled trial, cohort studies, or cross-sectional studies).

According to the search strategy, studies from January 1980 to March 2020 were included for relevant publications using keywords presented in Table 1. In addition, the keywords were searched with a Boolean phrase (Table 1).

Table 1. Search terms and following keywords for screening procedures.

\begin{tabular}{ccl}
\hline Search Term & \multicolumn{1}{c}{ Keywords } \\
\hline Football (population) & 1 & ("football" OR "soccer" OR "association football") \\
\hline Training load (dependent variable) & 2 & $\begin{array}{l}\text { ("training load" OR "external training load" OR } \\
\text { "workload" OR "internal training load" OR } \\
\text { "external load" OR "internal load") }\end{array}$ \\
\hline Periodization (independent variables) & $3 \begin{array}{l}\text { ("periodization" OR "schedule" OR "distribution" OR } \\
\text { "week" OR "microcycle" OR "mesocycle" OR "season } \\
\text { phase") AND ("in-season" OR "pre-season" OR } \\
\text { "preparation" OR "off-season" OR "post-season") }\end{array}$ \\
\hline Boolean search phrase (final search) & 4 & 1 AND (2 OR 3) \\
\hline
\end{tabular}

The literature search was accessed during February and March 2020. The search strategy was independently conducted by one review author and checked by a second author. Discrepancies between the authors in the study selection were solved with support of a third reviewer. The authors did not prioritize authors or journals. 


\subsection{Selection Criteria}

The included studies in the present review followed these inclusion criteria: (1) training load monitoring studies with adult and youth football players of both sexes; (2) studies with screening procedures based on internal and/or external load measures; (3) only studies that included the training load quantification of gross and temporal demands in complete/full training sessions (with or without match-play load); (4) observational prospective cohort, case-control, and/or cross sectorial design study including at least one week of monitoring; (5) studies of human physical and physiological performance in Sport Science and as scope; (6) original article published in a peer-review journal; (7) full text available in English; and (8) article reported sample and screening procedures (e.g., data collection, study design, instruments, and the outcomes).

The exclusion criteria were: (1) training load-based studies from team sport or football code population (e.g., Australian Football, Gaelic Football, Union, and/or Seven Rugby); (2) studies that monitored only match-play load; (3) participants aged $<13$ years and a match format other than 11-a-side football; (4) studies with screening procedures focused on biochemical loading, well-being, and/or injury intervention protocols; (5) studies that included the training load quantification based on field based test and laboratory test; (6) studies that included less than a week of monitoring and experimental trials or study cohort intervention with control group (pre- and post-) to evaluate the effect of a specific training method/program (e.g., small sided games, high intensity interval training, simulated games, or individualized approach); (7) others research areas and nonhuman participants; (8) articles with bad quality in the description of study sample and screening procedures (e.g., data collection, study design, instruments, and the measures) according to the strengthening the reporting of observational studies in epidemiology (STROBE) statement; and (9) reviews, abstract/papers conference, surveys, opinion pieces, commentaries, books, periodicals, editorials, case studies, non-peer-reviewed text, or Master's and/or doctoral thesis.

The search was limited to original articles published online until December 2020. Duplicated articles were identified and eliminated prior to application of the selection criteria (inclusion and exclusion). Titles and abstracts were initially selected and excluded according to selection criteria. The selection of full texts was based on a selection to determine the final status: inclusion or exclusion. Disagreements were resolved through discussion between two authors, or via a third researcher if required. Secondary-sourced articles considered relevant and with the same screening procedures were added.

\subsection{Quality Assessment}

The methodological quality was assessed using STROBE statement by two authors $[43,44]$. This checklist was used in previous reviews due their accuracy in the reporting of observational studies' cohorts, case-control, and cross-sectional studies [45,46]. The studies were classified as high-quality when missing fewer than three criteria of the STROBE checklist, while low-quality studies were defined as studies missing three or more criteria [45]. It included 22 items: title of the article and abstract interlinked (item 1), introduction (items 2 and 3), methods (items 4 to 12), results (items 13 to 17), discussion (items 18 to 21), and any other information (item 22). Four items were specific to the study design: participants (item 6), variables (item 12), descriptive data (item 14), and outcome data (item 15). The quality assessment was based on the attribution of one point for each checklist item if the criteria were evaluated as being complete (1 point), partial ( 0.5 points), or incomplete ( 0 points). The sum of the total points counted was divided by the maximum possible ( 22 items). Each author performed the classification independently with subsequent inter-observer reliability analysis:Kappa index (0.93; 90\%) and confidence interval (CI): 0.92-0.95).

\subsection{Study Coding and Data Extraction}

The data extractions from the included articles were performed according to: (1) summary measures describing construct, measure, measurement, thresholds, and/or metric 
formula with included article reference and further reading (Table 2); (2) subject and study characteristics according publication date, study design, completive level and standard, sample (N), and sex and anthropometric characteristics (stature and body mass) (Table 3); (3) methodological approaches: observations sample (monitoring period, training sessions recorded, trainings/week, training mode, and number of match-play), training load measures/metrics (internal and external load), and device specification (manufacturer model) (Table 4); (4) main findings: study purpose, periodization design, independent variables, findings, practical applications, and future directions. Data reporting were extracted according study purpose, periodization structure, independent variable, findings, and practical applications.

Table 2. Summary of measure and measurements in the included articles.

\begin{tabular}{|c|c|c|c|c|c|}
\hline Construct & Measure & Measurement & Thresholds and/or Metric Formula & Reference & $\begin{array}{l}\text { Further } \\
\text { Reading }\end{array}$ \\
\hline \multirow{13}{*}{$\begin{array}{l}\text { Internal } \\
\text { Load }\end{array}$} & \multirow{9}{*}{ Heart Rate } & \multirow{3}{*}{$\% \mathrm{HR}_{\max }$} & $\begin{array}{l}\text { Zone } 1: \leq 75 \% \mathrm{HR}_{\max } ; \text { zone } 2: 75-84.9 \% \mathrm{HR}_{\max } ; \text { zone } 3 \text { : } \\
\text { 85-89.9\% HR } \max \text {; zone } 4: \geq 90 \% \mathrm{HR}_{\max }\end{array}$ & {$[47,48]$} & [49] \\
\hline & & & $\begin{array}{c}\text { Zone } 1: \leq 75 \% \mathrm{HR}_{\max } ; \text { zone } 2: 75-84.9 \% \mathrm{HR}_{\max } ; \text { zone } 3 \text { : } \\
\text { 85-89.9\% } \mathrm{HR}_{\max } ; \text { zone } 4: \geq 90 \% \mathrm{HR}_{\max }\end{array}$ & {$[50]$} & {$[51]$} \\
\hline & & & $\begin{array}{l}\text { Zone 1: } 50-60 \% \mathrm{HR}_{\max } \text {; zone } 2: 60-70 \% \mathrm{HR}_{\max } \\
\text { zone 3: } 70-80 \% \mathrm{HR}_{\max } \text {; zone } 4: 80-90 \% \mathrm{HR}_{\max } \\
\text { zone 5: } 90-100 \% \mathrm{HR}_{\max }\end{array}$ & [52-61] & {$[62-64]$} \\
\hline & & $\mathrm{LT}_{\text {zone }}$ & $\begin{array}{l}\text { zone } 1 \text { : <LT; zone } 2 \text { : between } \mathrm{LT} \text { and AT; zone } 3 \text { : >AT } \\
(\mathrm{k}=1 \text { for zone } 1 ; \mathrm{k}=2 \text { for zone } 2, \text { and } \mathrm{k}=3 \text { for zone } 3)\end{array}$ & {$[52,54]$} & {$[63]$} \\
\hline & & $\begin{array}{l}\text { Bannister } \\
\text { TRIMP }\end{array}$ & $\begin{array}{c}\mathrm{D} \times\left(\Delta \mathrm{HR}_{\text {ratio }}\right) \times\left(0.64 \times \mathrm{e}^{\mathrm{b} \times \mathrm{HRB}}\right) \\
\left(\mathrm{D}=\left(\Delta \mathrm{HR}_{\text {ratio }}\right)\left[\left(\mathrm{HR}_{\mathrm{TS}}-\mathrm{HR}_{\mathrm{B}}\right) /\left(\mathrm{HR}_{\max }-\mathrm{HR}_{\mathrm{B}}\right)\right]\right) \\
\text { weighting factor }(\mathrm{k})=1.62(\text { females }) ; 1.92(\text { males })\end{array}$ & $\begin{array}{l}{[50,52,54} \\
57,58]\end{array}$ & {$[63]$} \\
\hline & & Edward's TL & $\begin{array}{c}\mathrm{D}(\text { zone } 1) \times 1+\mathrm{D}(\text { zone } 2) \times 2+\mathrm{D}(\text { zone } 3) \times 3+\mathrm{D} \\
(\text { zone } 4) \times 4+\mathrm{D}(\text { zone } 5) \times 5\end{array}$ & $\begin{array}{l}{[52,54,57,} \\
\quad 65]\end{array}$ & {$[66]$} \\
\hline & & $\begin{array}{c}\text { Lucia's } \\
\text { TL/LT } \\
\text { zone } \mathrm{TL}\end{array}$ & $\mathrm{D}($ zone 1$) \times 1+\mathrm{D}($ zone 2$) \times 2+\mathrm{D}($ zone 3$) \times 3$ & {$[52,54]$} & {$[66,67]$} \\
\hline & & $\begin{array}{l}\text { Stagno } \\
\text { TL/TRIMP } \\
\text { MOD }\end{array}$ & $\begin{array}{l}\left.\left[\left(\mathrm{HR}_{\mathrm{TS}}-\mathrm{HR}_{\mathrm{B}}\right) /\left(\mathrm{HR}_{\max }-\mathrm{HR}_{\mathrm{B}}\right)\right]\right) \\
\text { weighting factor }=0.1225 \mathrm{e}^{3.9434 \mathrm{x}}\end{array}$ & {$[50]$} & {$[51]$} \\
\hline & & HR-TL & $\sum$ (time $(\min )$ spent in zone $\times$ numerical factor of zone) & [56] & [68] \\
\hline & \multirow{4}{*}{$\begin{array}{l}\text { Perceived } \\
\text { Exertion }\end{array}$} & sRPE & $\mathrm{RPE} \times \mathrm{D}$ & $\begin{array}{c}{[52,54-} \\
59,69-72]\end{array}$ & {$[64,73-75]$} \\
\hline & & $\begin{array}{l}\text { sRPEresp TL/ } \\
\text { sRPEmusc TL }\end{array}$ & $\mathrm{sRPE} \times \mathrm{D}$ & [71] & {$[76,77]$} \\
\hline & & Fatigue score & $\begin{array}{c}\text { Seven-point scale: training exertion, sleep quality, } \\
\text { muscle soreness, infection/illness, concentration, } \\
\text { training efficiency, anxiety/irritability, and } \\
\text { general stress. }\end{array}$ & [69] & [78] \\
\hline & & $\mathrm{HI}$ & Fatigue, stress muscle soreness, and quality sleep. & [72] & [79] \\
\hline
\end{tabular}


Table 2. Cont

\begin{tabular}{|c|c|c|c|c|c|}
\hline Construct & Measure & Measurement & Thresholds and/or Metric Formula & Reference & $\begin{array}{l}\text { Further } \\
\text { Reading }\end{array}$ \\
\hline \multirow{20}{*}{$\begin{array}{l}\text { External } \\
\text { load }\end{array}$} & \multirow{10}{*}{$\begin{array}{l}\text { Distance } \\
\text { and speed }\end{array}$} & \multirow{10}{*}{$\begin{array}{l}\text { Speed zones/ } \\
\text { thresholds }\end{array}$} & $\begin{array}{c}\text { Zone 1: 0-6.9 } \mathrm{km} \times \mathrm{h}^{-1} \text {; zone 2: } 7.0-9.9 \mathrm{~km} \times \mathrm{h}^{-1} \\
\text { zone 3: } 10.0-12.9 \mathrm{~km} \times \mathrm{h}^{-1} \text {; zone } 4: 13-15.9 \mathrm{~km} \times \mathrm{h}^{-1} \\
\text { zone 5: } 16-17.9 \mathrm{~km} \times \mathrm{h}^{-1} \text {; and zone } 6: \geq 18.0 \mathrm{~km} \times \mathrm{h}^{-1} \\
\text { (sprints). }\end{array}$ & {$[47,48]$} & {$[80,81]$} \\
\hline & & & $\begin{array}{c}\text { Walking/jogging: }<10.8 \mathrm{~km} \times \mathrm{h}^{-1} \\
\text { HSR: } \geq 20.9 \mathrm{~km} \times \mathrm{h}^{-1} ; \text { SPR: }>24.1 \mathrm{~km} \times \mathrm{h}^{-1} .\end{array}$ & {$[53,82,83]$} & $\begin{array}{l}{[32,40,84-} \\
86]\end{array}$ \\
\hline & & & $\begin{array}{c}\text { Standing: } 0-0.6 \mathrm{~km} \times \mathrm{h}^{-1} \text {; walking: } 0.7-7.1 \mathrm{~km} \times \mathrm{h}^{-1} \text {; } \\
\text { jogging: } 7.2-14.3 \mathrm{~km} \times \mathrm{h}^{-1} \text {; running: } \\
\text { 14.4-19.7 } \mathrm{km} \times \mathrm{h}^{-1} ; \mathrm{HSR}: 19.8-25.1 \mathrm{~km} \times \mathrm{h}^{-1} \\
\text { SPR: }>25.1 \mathrm{~km} \times \mathrm{h}^{-1}\end{array}$ & [87-91] & {$[92,93]$} \\
\hline & & & $\begin{array}{c}\text { Running: } 11.4-18.9 \mathrm{~km} \times \mathrm{h}^{-1} \text {; HSR: } \\
\text { 15.0-18.9 km } \times \mathrm{h}^{-1} ; \text { SPR: }>19.0 \mathrm{~km} \times \mathrm{h}^{-1} \text {. }\end{array}$ & {$[58]$} & [94] \\
\hline & & & $\begin{array}{l}\text { Walking: 0-6.9 } \mathrm{km} \times \mathrm{h}-1 \text {; jogging: } 7.0-13.9 \mathrm{~km} \times \mathrm{h}^{-1} \text {; } \\
\text { Running: } 14.0-20.0 \mathrm{~km} \times \mathrm{h}^{-1} \text {; SPR: }>20.0 \mathrm{~km} \times \mathrm{h}^{-1} \text {. }\end{array}$ & {$[88,89,95]$} & [96] \\
\hline & & & $\begin{array}{l}\text { Low-speed running: }<14.4 \mathrm{~km} \times \mathrm{h}^{-1} ; \text { HSR: }>19.8 \text {; } \\
\text { SPR: }>25.2 \mathrm{~km} \times \mathrm{h}^{-1}\end{array}$ & $\begin{array}{l}{[57,70,91} \\
97-99]\end{array}$ & $\begin{array}{l}{[11,100-} \\
104]\end{array}$ \\
\hline & & & $\begin{array}{l}\text { Low-speed running: }<14 \mathrm{~km} \times \mathrm{h}^{-1} \\
\text { HSR: } 14.4 \mathrm{~km} \times \mathrm{h}^{-1} ; \text { HSR: } 19.8-25.2 \mathrm{~km} \times \mathrm{h}^{-1}\end{array}$ & {$[102,105]$} & [104] \\
\hline & & & HSR: $>19 \mathrm{~km} \times \mathrm{h}^{-1}$. & {$[72]$} & [104] \\
\hline & & & HSR: $>16 \mathrm{~km} \times \mathrm{h}^{-1}$. & {$[61]$} & [85] \\
\hline & & & $\begin{array}{l}\text { Standing/walking: } 0-7.2 \mathrm{~km} \times \mathrm{h}^{-1} \text {; low intensity } \\
\text { running: } 7.3-14.3 \mathrm{~km} \times \mathrm{h}^{-1} ; \text { moderate intensity } \\
\text { running: } 14.4-21.5 \mathrm{~km} \times \mathrm{h}^{-1} ; \mathrm{HSR}: 19.8-25.1 \mathrm{~km} \times \mathrm{h}^{-1} \text {; } \\
\text { very } \mathrm{HSR}>25.1 \mathrm{~km} \times \mathrm{h}^{-1} \text {. }\end{array}$ & [106-108] & {$[30,40,109]$} \\
\hline & \multirow{6}{*}{ Acceleration } & \multirow{6}{*}{$\begin{array}{l}\text { Acceleration } \\
\text { zones / } \\
\text { thresholds }\end{array}$} & $\begin{array}{c}\text { Low: } 1-2 \mathrm{~m} \times \mathrm{s}^{-2} ; \text { Moderate: } 2-3 \mathrm{~m} \times \mathrm{s}^{-2} \\
\text { High: }>3 \mathrm{~m} \times \mathrm{s}^{-2}\end{array}$ & {$[53]$} & [85] \\
\hline & & & ACC: $>2.5 \mathrm{~m} \times \mathrm{s}^{-2} ;$ DEC: $<2.5 \mathrm{~m} \times \mathrm{s}^{-2}$. & [91] & {$[40]$} \\
\hline & & & ACC: $>2 \mathrm{~m} \times \mathrm{s}^{-2}$. & {$[99,110]$} & {$[111]$} \\
\hline & & & ACC/DEC: $>3 \mathrm{~m} \times \mathrm{s}^{-2}$. & {$[61,82,98]$} & [86] \\
\hline & & & ACC: $>4 \mathrm{~m} \times \mathrm{s}^{-2}$. & {$[108]$} & {$[100]$} \\
\hline & & & $\begin{array}{c}\text { ACC: medium }\left(1.5-3.0 \mathrm{~m} \times \mathrm{s}^{-2}\right) ; \text { high }\left(>3.0 \mathrm{~m} \times \mathrm{s}^{-2}\right) \\
\text { DEC: } \\
\text { hedium }\left(-1.5 \text { to }-3.0 \mathrm{~m} \times \mathrm{s}^{-2}\right) \\
\text { high }\left(<-3.0 \mathrm{~m} \times \mathrm{s}^{-2}\right)\end{array}$ & {$[82]$} & {$[102,111]$} \\
\hline & \multirow{4}{*}{$\begin{array}{l}\text { Accele- } \\
\text { rometry }\end{array}$} & $\begin{array}{c}\text { Body impacts / } \\
\text { body load }\end{array}$ & $\begin{array}{l}\text { Zone 1: } 5.0-6.0 \mathrm{~g} \text {; zone } 2: 6.1-6.5 \mathrm{~g} \text {; zone } 3: 6.5-7.0 \mathrm{~g} \text {; } \\
\text { zone 4: } 7.1-8.0 \mathrm{~g} \text {; zone 5: } 8.1-10.0 \mathrm{~g} \text {; zone } 6: \geq 10.1 \mathrm{~g} \text {. }\end{array}$ & $\begin{array}{l}{[47,48,98,} \\
99]\end{array}$ & [112-114] \\
\hline & & Player load & $\sqrt{\left(a_{x 1}-a_{y-1}\right)^{2}+\left(a_{y 1}-a_{y-1}\right)^{2}+\left(a_{z 1}-a_{z-1}\right)^{2}} / 100$ & $\begin{array}{l}{[53,88,89,} \\
115]\end{array}$ & {$[10,116]$} \\
\hline & & Player load & $\left(a_{x 1}-a_{y-1}\right)^{2}+\left(a_{y 1}-a_{y-1}\right)^{2}+\left(a_{z 1}-a_{z-1}\right)^{2}$ & {$[57]$} & {$[117]$} \\
\hline & & $\begin{array}{l}\text { Dynamic-stress } \\
\text { load }\end{array}$ & $\sum$ (body load for each zone $\times$ body mass) & [118] & [119] \\
\hline
\end{tabular}


Table 2. Cont.

\begin{tabular}{|c|c|c|c|c|c|}
\hline Construct & Measure & Measurement & Thresholds and/or Metric Formula & Reference & $\begin{array}{l}\text { Further } \\
\text { Reading }\end{array}$ \\
\hline \multirow{6}{*}{$\begin{array}{l}\text { Ratios / } \\
\text { scores }\end{array}$} & \multirow{3}{*}{$\begin{array}{l}\text { Ratio/scores } \\
\text { (Weekly TL) }\end{array}$} & Work: rest ratio & $\begin{array}{c}\text { High to very high: }>16 \mathrm{~km} \times \mathrm{h}^{-1} \\
\text { moderate: } 10.0-15.9 \mathrm{~km} \times \mathrm{h}^{-1} \text {; low intensity: } \\
7.0-9.9 \mathrm{~km} \times \mathrm{h}^{-1} \text {; very low intensities: } 0-6.9 \mathrm{~km} \times \mathrm{h}^{-1} \\
\text { (normalized for each } 100 \mathrm{~m})\end{array}$ & [47] & [80] \\
\hline & & Work: rest ratio & $\begin{array}{l}\text { WRR: distance covered at a speed } \geq 4 \mathrm{~km} \times \mathrm{h}^{-1} \text { period } \\
\text { of activity or work divided by the distance covered at a } \\
\text { speed }<3.9 \mathrm{~km} \times \mathrm{h}^{-1} \text {; period of recovery or rest); } \\
\text { FEHS } \geq 18 \mathrm{~km} \times \mathrm{h}^{-1} \text {; FESS } \geq 21 \mathrm{~km} \times \mathrm{h}^{-1} \text {. }\end{array}$ & [115] & [119] \\
\hline & & THIA (\%) & $\sum$ (MSR, HSR and SPR) & [107] & $\begin{array}{l}\text { originally } \\
\text { proposed by } \\
\text { Rago et al. } \\
\text { [107] }\end{array}$ \\
\hline & \multirow{3}{*}{$\begin{array}{l}\text { Ratio/scores } \\
\text { (Weekly TL } \\
\text { and ML) }\end{array}$} & TMr & (Weekly load)/(Match load) & [95] & $\begin{array}{l}\text { originally } \\
\text { proposed by } \\
\text { Clemente } \\
\text { et al. [95] }\end{array}$ \\
\hline & & $\begin{array}{l}\text { Session volume } \\
\text { score }\end{array}$ & (Volume Metric $\mathrm{x}_{1}, \mathrm{x}_{2}, \mathrm{x}_{3}, \mathrm{x}_{4}$ of MD (\%)/4) & [97] & $\begin{array}{l}\text { originally } \\
\text { proposed } \\
\text { by Owen } \\
\text { et al. [97] }\end{array}$ \\
\hline & & $\begin{array}{l}\text { Session } \\
\text { intensity } \\
\text { score }\end{array}$ & (Intensity Metric $\mathrm{x}_{1}, \mathrm{x}_{2}, \mathrm{x}_{3}, \mathrm{x}_{4}$ of MD $\left.(\%) / 4\right)$ & [97] & $\begin{array}{l}\text { originally } \\
\text { proposed } \\
\text { by Owen } \\
\text { et al. [97] }\end{array}$ \\
\hline \multirow{2}{*}{$\begin{array}{l}\text { Energy } \\
\text { cost and } \\
\text { metabolic } \\
\text { power }\end{array}$} & \multirow{2}{*}{$\begin{array}{l}\text { Equivalent- } \\
\text { estimation }\end{array}$} & $\mathrm{EC}$ & $\begin{array}{c}\mathrm{EC}=155.4 \times 155.4 \times \mathrm{ES}^{4} \times 155.4 \times \mathrm{ES}^{3} \times 155.4 \times \mathrm{ES}^{2} \\
\times 155.4 \times \mathrm{ES} \times \mathrm{EM} \times \mathrm{KT}\end{array}$ & {$[90]$} & {$[84,85]$} \\
\hline & & $P_{\text {met }}$ & HP: $20-35 \mathrm{~W} \times \mathrm{kg}^{-1} ; \mathrm{EP}: 35-55 \mathrm{~W} \times \mathrm{kg}^{-1} ;:>55 \mathrm{~W} \times \mathrm{kg}$ & $\begin{array}{c}{[82,83,} \\
118]\end{array}$ & {$[84,85]$} \\
\hline
\end{tabular}

$\triangle \mathrm{HR}$ - HR variation; ACC—acceleration; AT—anaerobic threshold; D—duration; DEC—deceleration; EC—energy cost; EM-equivalent body mass; EP—elevated power; ES — equivalent slope; FEHS—frequency of efforts at high speed $\left(\geq 18 \mathrm{~km} \times \mathrm{h}^{-1}\right)$; FESS—frequency of efforts at sprint speed $\left(\geq 21 \mathrm{~km}^{-1}\right.$ ) ; HI-Hooper Index; HP-high power; HR-heart rate; $\mathrm{HR}_{\mathrm{B}}$ —basal heart rate; $\mathrm{HR}_{\max }$-maximum heart rate; $\mathrm{HR}_{\mathrm{TL}}$ - heart rate training load; $\mathrm{HR}_{\mathrm{TS}}$ —average training session heart rate; $\mathrm{HSR}$ — high speed running; $\mathrm{K}$ —coefficient relative; $\mathrm{KT}$ —constant;

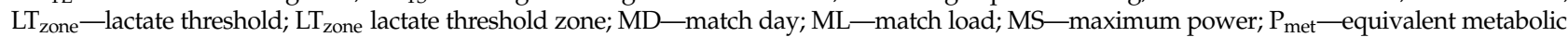
power; RPE—ratings of perceived exertion; SPR—sprinting; sRPE—sessions ratings of perceived exertion; sRPEmusc-TL—sessions ratings of muscular training load; sRPEres-TL—sessions ratings of respiratory training load; THIA—total high-intensity activity; TL—training load; $\mathrm{TMr}$ - training/match ratio; TRIMP—-training impulse; TRIMP $\mathrm{MOD}$ — modified training impulse; WRR—work:rest ratio.

The outcome measures and the statistical procedures used in the included references were inconsistent between studies, making it impossible to group data and perform the meta-analysis. Characterization of participants is reported as mean \pm standard deviation, $\mathrm{CI}$, and effect size (ES) wherever possible. In order to clarify the variety of internal and external load measures used in the included studies, Table 2 consolidates the thresholds used by the authors to calculate metric formulas. In addition, the references correspond to the article reviewed and their construct, measure, and methods. The further reading includes the original references used by the reviewed studies to ensure the methodological procedures.

\section{Results}

\subsection{Search Results and Study Selection}

A total of 7972 titles were collected through four database searches $($ WoS $=1204$; Pub-Med = 869; SCOPUS = 5083; and SportDiscus $=816$ ). No articles were identified from additional sources as a potentially relevant and unidentified research strategy. A total of 188 duplicate records were removed, and 884 articles were removed based on the title and abstract according to inclusion and exclusion criteria. A total of 146 full-text articles were 
assessed for eligibility and 116 were removed. The reasons for exclusion were: (1) studies not related systematic review purpose $(n=53)$; (2) studies not related to football player's topic $(n=13)$; (3) studies related only to match load/demands $(n=7)$; (4) studies with screening procedures based on biochemical loading, well-being, and/or injury intervention protocols $(n=15)$; (5) studies that included field-based test and laboratory test for training load quantification $(n=11)$; (6) editorials, commentaries, and literature reviews $(n=12)$; (7) case studies $(n=3)$; $(8)$ conference abstract/papers $(n=1)$; and (9) other language $(n=1)$. After screening procedures, 36 articles were included in the present systematic review. A detailed representation of the screening procedures is depicted with a PRISMA flow diagram in Figure 1.
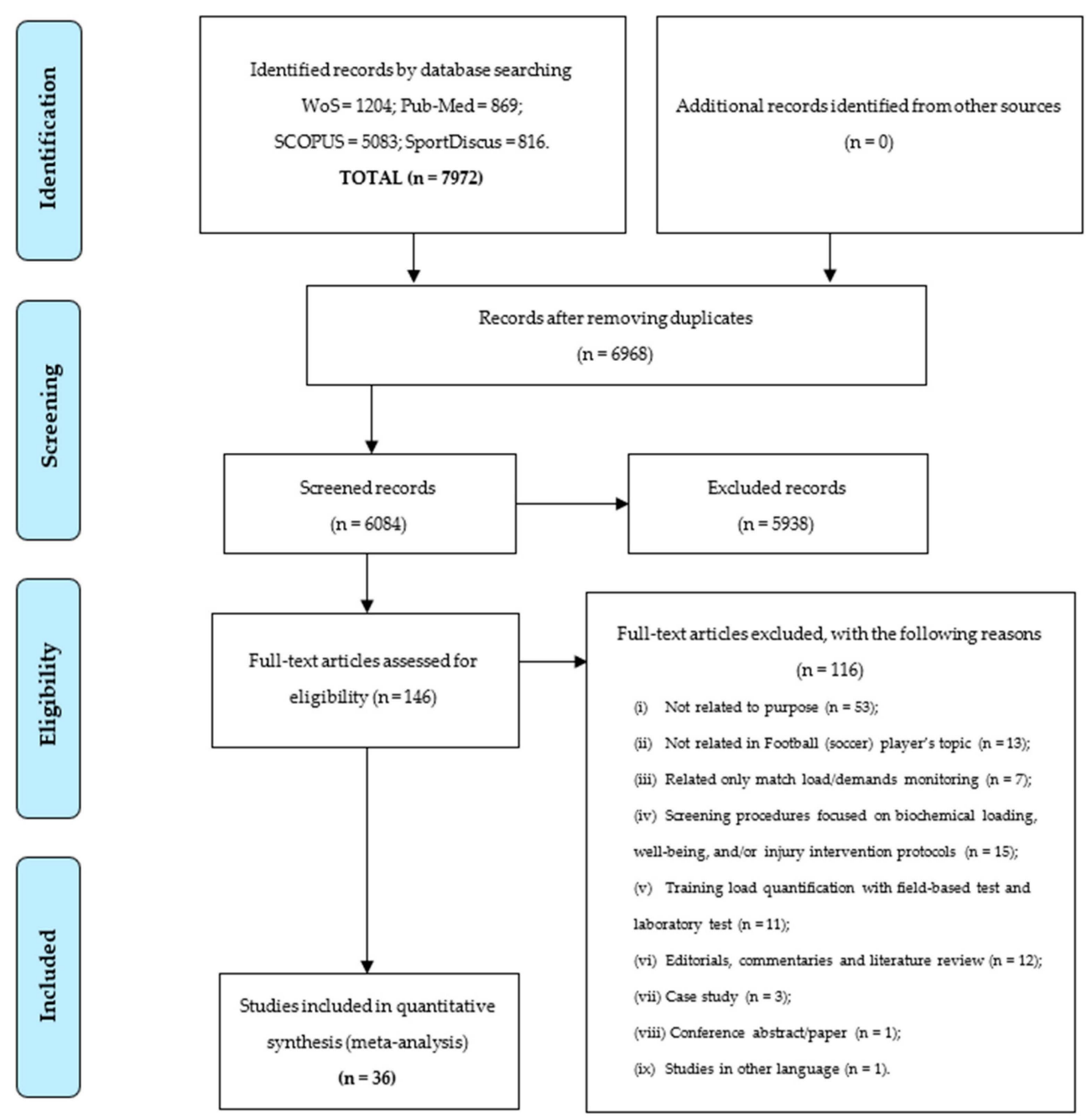

Figure 1. Preferred reporting item for systematic reviews and meta-analyses (PRISMA) flow diagram.

\subsection{Participant Characteristics}

The reviewed articles were published between 2004-2020. All included articles presented a quasi-experimental approach based on observational and prospective cohort design. The included studies were performed in elite/professional $(n=32)$, pre-elite $(n=3)$, and amateur $(n=1)$ football. One article did not specify the participants' competitive level. Twenty-seven articles focused on adult player population and nine on youths. The geographic location of the populations studied in reviewed studies were Australia $(n=1)$, Brazil $(n=1)$, France $(n=3)$, Italy $(n=1)$, Korea $(n=1)$, Norway $(n=2)$, Portugal $(n=6)$, Spain $(n=5)$, Swiss $(n=1)$, The Netherlands $(n=3)$, and the United Kingdom $(n=11)$. Four studies did not specify the geographic location and one study was sampled in an European population. 
The study samples ranged between 13-160 participants. All articles were performed on male football players, except one on female players. A total of 1317 (1302 men and 15 women) adult and youth football players were analyzed for this systematic review. The mean and standard deviation for age and anthropometric data (weight and height) in the included studies was $22.71 \pm 4.37$ years, $74.13 \pm 6.77 \mathrm{~kg}$, and $1.71 \pm 0.05 \mathrm{~m}$, respectively. Table 3 provides a summary of the participants demographics.

\subsection{Quality Assessment}

In the evaluation of methodological quality, the mean quality score and standard deviation of all the included studies was $0.79 \pm 0.06$ (Table 3). One study $(3.33 \%)$ was classified with a quality score of 0.65 . Twenty studies (56.67\%) were classified between 0.7 and 0.8 , whereas fifteen studies $(40.00 \%)$ had a quality score between 0.8 and 0.9 . None of the reviewed studies had the maximum score (1.0) or below 0.5 (min: 0.65 ; max: 0.89 ).

\subsection{Data Organization}

The results are presented in the following three sub topics: (1) analysis of the intra- and inter-individual accumulative training load distribution within one week (microcycle), weeks (mesocycle), and/or season phases; (2) analysis of the intra- and inter-individual accumulative training load and match load distribution within one week (microcycle), weeks (mesocycle), and/or season phases; and (3) analysis of the relationships between internal and external load measures in the accumulative training load quantification. Observation samples were collected from 17 to 2981 training sessions and varied between 3 and 6 trainings per week. Twenty studies analyzed training data and ten articles integrate training data with match load. The monitoring period in the included studies ranged from 3 to 43 weeks. The included match-play varied from 1 to 623 games. Four studies did not describe the number of observed weeks and six studies did not describe training sessions. Eleven articles evaluated training load with internal load measures; fourteen articles included only external load measures; and eleven studies analyzed internal and external measures.

The training load quantification in the included studies were based on internal and external load measures/metrics. Twelve articles analyzed only internal load measures, twelve articles evaluated the external load, and twelve studies assessed both measures. The studies that quantified only internal load were based on summated zones of maximum heart rate $\left(\mathrm{HR}_{\max }\right)(n=10)$, and training impulse $(n=11)$. Banister TRIMP was reported in four studies, Edwards TRIMP in five studies, and lactate threshold (LTzone) and modified Stagno training impulse (TRIMP ${ }_{\text {MOD }}$ ) were both required in one study. Still, external load measures were quantified with distance and covered distance in different speed zones $(n$ $=27)$, acceleration and deceleration (ACC/DEC) $(n=13)$, accelerometer metrics $(n=11)$, metabolic power output $(n=4)$, and ratios/scores $(n=6)$.

The methodological approaches of the reviewed articles were based on short-telemetry systems $(n=12)$, GPS systems $(n=25)$, MEMS $(n=18)$, LPM systems $(n=3)$, and multiplecamera systems (i.e., Prozone ${ }^{\circledR}$, Leeds, UK) $(n=3)$. Additionally, the internal load measures were reported with perceived exertion scales (i.e., Borg's Category-Ratio scale, Hooper Index, and Fatigue Questionnaire) ( $n=16)$. The internal load based on heart rate (HR) measures were reported in twelve of the included studies $(n=12)$; with $1 \mathrm{~Hz}$ telemetry system and five studies with $5 \mathrm{~Hz}$. Two studies did not specify the telemetry range in the methodology description. Furthermore, internal load based on perceived exertion by Borg's Category-Ratio scale was presented in fifteen studies. One study assessed perceived exertion with the Hooper Index and one other with the Fatigue Questionnaire. Regarding systems tracking, $5 \mathrm{~Hz}$ GPS, $10 \mathrm{~Hz}$ GPS, and $15 \mathrm{~Hz}$ GPS were used in one study, fifteen studies, and four studies, respectively. The $100 \mathrm{~Hz}$ MEMS integrated the GPS device and was reported in ten studies. The LPM system was reported only in one study.

The data organization respected the three main purposes of this systematic review. Table 4 presents the methodological approaches selected by the studies included in this review. 
Table 3. Summary characteristics of the participants' demographics recruited in the studies included in the systematic review and its quality score.

\begin{tabular}{|c|c|c|c|c|c|c|c|c|c|}
\hline Reference (Year) & Study Design & Population & $\begin{array}{c}\text { Competitive Level, } \\
\text { Country }\end{array}$ & Sample (N) & Sex & Age (y) & Stature & Body Mass (kg) & QS \\
\hline Abade et al. [47] & $\begin{array}{l}\text { Prospective } \\
\text { Cohort }\end{array}$ & Youth & Elite, Portugal & 151 & Male & $\begin{array}{l}\mathrm{U} 15(n=56): 14.0 \pm 0.2 \\
\mathrm{U} 17(n=66): 15.8 \pm 0.4 \\
\mathrm{U} 19(n=29): 17.8 \pm 0.6\end{array}$ & $\begin{array}{l}\mathrm{U} 15(n=56): 1.71 \pm 0.07 \\
\mathrm{U} 17(n=66): 1.76 \pm 0.06 \\
\mathrm{U} 19(n=29): 1.77 \pm 0.07\end{array}$ & $\begin{array}{l}\text { U15 }(n=56): 60.1 \pm 6.3 \\
\text { U17 }(n=66): 65.8 \pm 5.5 \\
\text { U19 }(n=29): 70.0 \pm 5.6\end{array}$ & 0.78 \\
\hline Akenhead et al. [53] & $\begin{array}{l}\text { Prospective } \\
\text { Cohort }\end{array}$ & Adult & Elite, UK & 33 & Male & $24.0 \pm 4.0$ & $1.83 \pm 0.05$ & $82 \pm 8.0$ & 0.87 \\
\hline Alexiou and Coutts [52] & $\begin{array}{c}\text { Prospective } \\
\text { cohort }\end{array}$ & Adult & Elite, Portugal & 15 & Female & $19.3 \pm 2.0$ & $1.69 \pm 0.05$ & $64.8 \pm 7.7$ & 0.83 \\
\hline Anderson et al. [106] & $\begin{array}{c}\text { Prospective } \\
\text { Cohort }\end{array}$ & Youth & Elite, UK & 19 & Male & $25.0 \pm 4.0$ & $1.78 \pm 0.06$ & $80.6 \pm 8.3$ & 0.74 \\
\hline Baptista et al. [97] & $\begin{array}{l}\text { Prospective } \\
\text { Cohort }\end{array}$ & Adult & Elite, Norway & 18 & Male & ND & ND & ND & 0.74 \\
\hline Brito et al. [69] & $\begin{array}{c}\text { Prospective } \\
\text { Cohort }\end{array}$ & Adult & Elite, France & 13 & Male & $18.6 \pm 0.5$ & $1.77 \pm 0.05$ & $70.0 \pm 7.3$ & 0.78 \\
\hline Campos-Vazquez et al. [50] & $\begin{array}{l}\text { Prospective } \\
\text { Cohort }\end{array}$ & Adult & Elite, Spain & 9 & Male & $26.7 \pm 4.5$ & $1.77 \pm 0.07$ & $74.5 \pm 5.7$ & 0.74 \\
\hline Casamichana et al. [115] & $\begin{array}{l}\text { Prospective } \\
\text { Cohort }\end{array}$ & Adult & Elite, Spain & 28 & Male & $22.9 \pm 4.2$ & $1.77 \pm 0.05$ & $73.6 \pm 4.4$ & 0.87 \\
\hline Clemente et al. [89] & $\begin{array}{l}\text { Prospective } \\
\text { Cohort }\end{array}$ & Adult & $\begin{array}{l}\text { Elite, Portugal and } \\
\text { The Netherlands }\end{array}$ & 29 & Male & $\begin{array}{l}\text { PT }(n=14): 19.21 \pm 1.05 \\
\text { NL }(n=15): 25.14 \pm 3.90\end{array}$ & $\begin{array}{l}\text { PT }(n=14): 1.80 \pm 0.06 \\
\text { NL }(n=15): 1.79 \pm 0.06\end{array}$ & $\begin{array}{l}\text { PT }(n=14): 74.07 \pm 6.21 \\
\text { NL }(n=15): 73.21 \pm 6.46\end{array}$ & 0.74 \\
\hline Clemente et al. [95] & $\begin{array}{l}\text { Prospective } \\
\text { Cohort }\end{array}$ & Adult & Elite, Portugal & 27 & Male & $24.9 \pm 3.5$ & $1.69 \pm 0.41$ & $71.6 \pm 18.7$ & 0.83 \\
\hline Clemente et al. [88] & $\begin{array}{l}\text { Prospective } \\
\text { Cohort }\end{array}$ & Youth & $\begin{array}{l}\text { Elite, Portugal and } \\
\text { The Netherlands }\end{array}$ & 89 & Male & $\begin{array}{c}\text { NL1 }(n=18): 25.39 \pm 4.82 \\
\text { NL2 }(n=24): 21.46 \pm 2.50 \\
\text { NL3 }(n=23): 23.00 \pm 3.70 \\
\text { PT }(n=24): 24.70 \pm 2.92\end{array}$ & $\begin{array}{l}\text { NL1 }(n=18): 1.84 \pm 0.05 \\
\text { NL2 }(n=24): 1.80 \pm 0.08 \\
\text { NL3 }(n=23): 1.84 \pm 0.06 \\
\text { PT }(n=24): 1.81 \pm 0.06\end{array}$ & $\begin{array}{l}\text { NL1 }(n=18): 77.29 \pm 4.73 \\
\text { NL2 }(n=24): 71.73 \pm 8.61 \\
\text { NL3 }(n=23): 74.50 \pm 6.90 \\
\text { PT }(n=24): 77.48 \pm 6.80\end{array}$ & 0.87 \\
\hline Clemente et al. [105] & $\begin{array}{l}\text { Prospective } \\
\text { Cohort }\end{array}$ & Adult & Elite, Europe * & 19 & Male & $26.5 \pm 4.3$ & $1.80 \pm 7.3$ & $75.6 \pm 9.6$ & 0.83 \\
\hline Coutinho et al. [47] & $\begin{array}{l}\text { Prospective } \\
\text { Cohort }\end{array}$ & Adult & Elite, Portugal & 151 & Male & $\begin{array}{l}\mathrm{U} 15(n=256): 14.0 \pm 0.2 \\
\mathrm{U} 17(n=66): 15.8 \pm 0.4 \\
\mathrm{U} 19(n=29): 17.8 \pm 0.6\end{array}$ & $\begin{array}{l}\mathrm{U} 15(n=56): 1.71 \pm 0.07 \\
\mathrm{U} 17(n=66): 1.76 \pm 0.06 \\
\mathrm{U} 19(n=29): 1.77 \pm 0.07\end{array}$ & $\begin{array}{l}\text { NL1 }(n=18): 77.29 \pm 4.73 \\
\text { NL2 }(n=24): 71.73 \pm 8.61 \\
\text { NL3 }(n=23): 74.50 \pm 6.90 \\
\text { PT }(n=24): 77.48 \pm 6.80\end{array}$ & 0.74 \\
\hline
\end{tabular}


Table 3. Cont

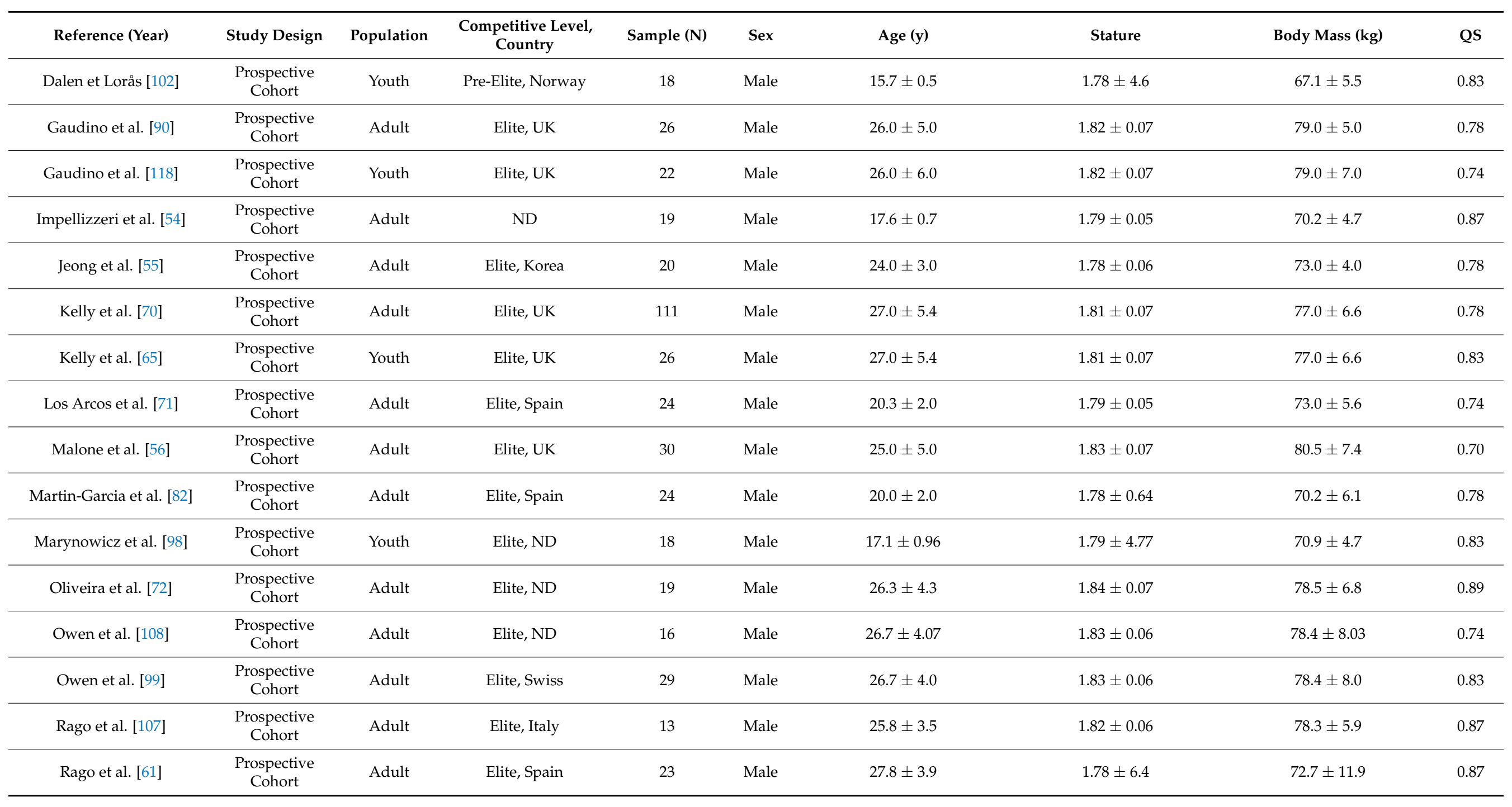


Table 3. Cont.

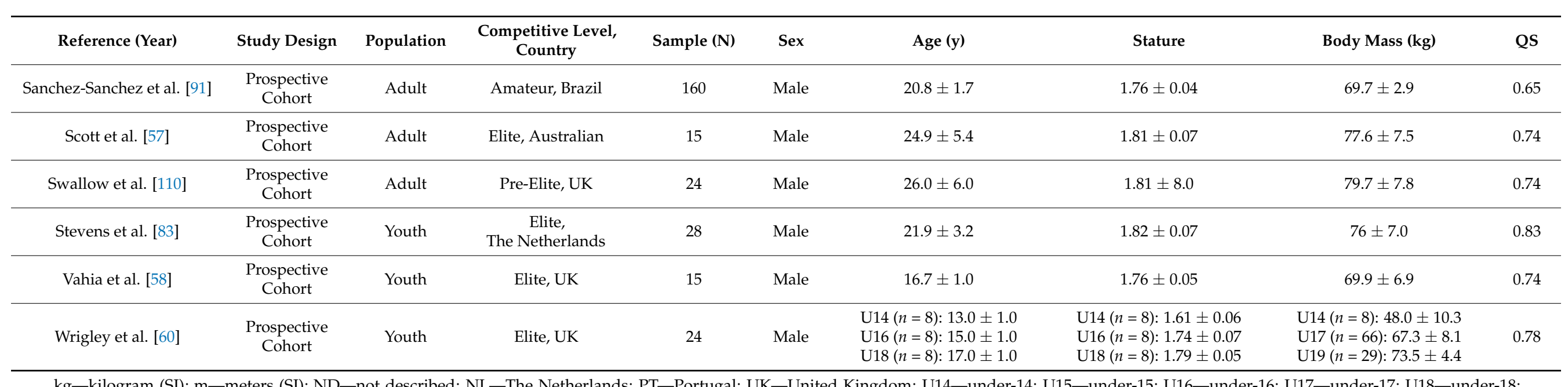

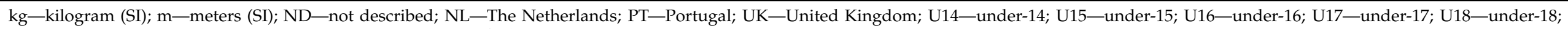

U19-under-19; QS-quality score. Note: * Country is not specified.

Table 4. Methodological approaches of included articles.

\begin{tabular}{|c|c|c|c|c|c|c|c|c|c|}
\hline \multirow{2}{*}{$\begin{array}{l}\text { Reference } \\
\text { (Year) }\end{array}$} & \multicolumn{5}{|c|}{ Observations Sample } & \multicolumn{2}{|c|}{ Training Load Measures/Metrics } & \multicolumn{2}{|c|}{$\begin{array}{c}\text { Device Specification } \\
\text { (Manufacturer Model and Specs) }\end{array}$} \\
\hline & $\begin{array}{l}\text { Monitoring } \\
\text { Period }\end{array}$ & $\begin{array}{l}\text { Training } \\
\text { Sessions }\end{array}$ & TS/Week & Sets & Match-Play & Internal Load & External Load & Internal Load & External Load \\
\hline Abade et al. [47] & 9 weeks & $38 \mathrm{TS}$ & $\begin{array}{c}4 \mathrm{TS} / \mathrm{wk} \\
(\sim 90 \mathrm{~min})\end{array}$ & 612 & ND & HR: $\% \mathrm{HR}_{\max }$ & $\begin{array}{l}\text { Distance and speed: TD } \\
\text { covered }(\mathrm{m}) \text {; relative } \\
\text { distance or pace } \\
\left(\mathrm{m} \times \mathrm{min}^{-1}\right) \text {; } \mathrm{D} \text { in different } \\
\text { speed zone }\left(\mathrm{km} \times \mathrm{h}^{-1}\right) ; \\
\text { and sprints }(\text { number and } \\
\text { time interval). } \\
\text { Accelerometry: absolute } \\
\text { and relative body } \\
\text { impacts }(\mathrm{g}) .\end{array}$ & $\begin{array}{c}5 \mathrm{~Hz} \text { short-range } \\
\text { telemetry system (Polar } \\
\text { Team System, } \\
\text { Kempele, Finland). }\end{array}$ & $\begin{array}{c}15 \text { Hz GPS and 100-Hz } \\
\text { MEMS (SPI-Pro X II, } \\
\text { GPSports, } \\
\text { Canberra, Australia). }\end{array}$ \\
\hline
\end{tabular}


Table 4. Cont.

\begin{tabular}{|c|c|c|c|c|c|c|c|c|c|}
\hline \multirow{2}{*}{$\begin{array}{l}\text { Reference } \\
\text { (Year) }\end{array}$} & \multicolumn{5}{|c|}{ Observations Sample } & \multicolumn{2}{|c|}{ Training Load Measures/Metrics } & \multicolumn{2}{|c|}{$\begin{array}{c}\text { Device Specification } \\
\text { (Manufacturer Model and Specs) }\end{array}$} \\
\hline & $\begin{array}{l}\text { Monitoring } \\
\text { Period }\end{array}$ & $\begin{array}{l}\text { Training } \\
\text { Sessions }\end{array}$ & TS/Week & Sets & Match-Play & Internal Load & External Load & Internal Load & External Load \\
\hline $\begin{array}{l}\text { Akenhead et al. } \\
\text { [53] }\end{array}$ & 12 weeks & $48 \mathrm{TS}$ & $5 \mathrm{TS} / \mathrm{wk}$ & 295 & $\begin{array}{l}1 \mathrm{MP} / \mathrm{wk} \\
(90 \mathrm{~min})\end{array}$ & HR: \%HR $\max$ & $\begin{array}{l}\text { Distance and speed: TD } \\
\text { covered }(\mathrm{m}) \text {; HSR } \\
\left(\mathrm{km} \times \mathrm{h}^{-1}\right) \text {; and SPR } \\
\left(\mathrm{km} \times \mathrm{h}^{-1}\right) \\
\text { Acceleration: } \mathrm{ACC}_{\mathrm{TOTAL}} \\
\left(\mathrm{m} \times \mathrm{s}^{-2}\right) \text { and DEC } \mathrm{TOTAL} \\
\quad\left(\mathrm{m} \times \mathrm{s}^{-2}\right)\end{array}$ & $\begin{array}{c}1 \mathrm{~Hz} \text { short-range } \\
\text { telemetry system (Team 2, } \\
\text { Polar Electro, } \\
\text { Kempele, Finland). }\end{array}$ & $\begin{array}{c}10 \mathrm{~Hz} \text { GPS and } 100-\mathrm{Hz} \\
\text { MEMS (Catapult MiniMaxx } \\
\text { S4, Firmware 6.7, } \\
\text { Melbourne, Australia). }\end{array}$ \\
\hline $\begin{array}{l}\text { Alexiou and } \\
\text { Coutts [52] }\end{array}$ & 16 weeks & $623 \mathrm{TS}$ & ND & ND & $623 \mathrm{MP}$ & $\begin{array}{l}\text { HR: Bannister } \\
\text { TL, Edward's } \\
\text { TL and } \\
\text { LTzone TL. }\end{array}$ & ND & $\begin{array}{c}1 \mathrm{~Hz} \text { short-range } \\
\text { telemetry system Polar } \\
\text { NV, Polar Electro, } \\
\text { Kempele, Finland). }\end{array}$ & ND \\
\hline $\begin{array}{c}\text { Anderson et al. } \\
{[87]}\end{array}$ & 3 weeks & $10 \mathrm{TS}$ & $5 \mathrm{TS} / \mathrm{wk}$ & 145 & $6 \mathrm{MP}$ & ND & $\begin{array}{c}\text { Distance and speed: TS } \\
\text { duration }(\mathrm{min}) \text {; TD covered } \\
(\mathrm{m}) \text {; AvS }\left(\mathrm{m} \times \mathrm{min}^{-1}\right) \text {; and } \\
\mathrm{D} \text { in different speed zones } \\
\left(\mathrm{km} \times \mathrm{h}^{-1}\right)\end{array}$ & ND & $\begin{array}{l}10 \text { Hz GPS (Viper pod 2, } \\
\text { STATSports }{ }^{\circledR}, \text { Newry, } \\
\text { Northern Ireland) and } \\
\text { semi-automatic } \\
\text { multiple-camera system } \\
\text { (Prozone Sports Ltd., Leeds, } \\
\text { United Kingdom). }\end{array}$ \\
\hline $\begin{array}{c}\text { Anderson et al. } \\
{[106]}\end{array}$ & 39 weeks & $181 \mathrm{TS}$ & ND & 2182 & $7 \mathrm{MP}$ & ND & $\begin{array}{l}\text { Distance and speed: TS } \\
\text { duration (min); TD covered } \\
(\mathrm{m}) \text {; and } \mathrm{D} \text { in different } \\
\text { speed zones }\left(\mathrm{km} \times \mathrm{h}^{-1}\right) \text {. }\end{array}$ & ND & $\begin{array}{l}10 \text { Hz GPS (Viper pod 2, } \\
\text { STATSports, Northern } \\
\text { Ireland) and } \\
\text { semi-automatic } \\
\text { multiple-camera system } \\
\text { (Prozone Sports Ltd. }{ }^{\circledR}, \\
\text { Leeds, United Kingdom). }\end{array}$ \\
\hline
\end{tabular}


Table 4. Cont.

\begin{tabular}{|c|c|c|c|c|c|c|c|c|c|}
\hline \multirow{2}{*}{$\begin{array}{l}\text { Reference } \\
\text { (Year) }\end{array}$} & \multicolumn{5}{|c|}{ Observations Sample } & \multicolumn{2}{|c|}{ Training Load Measures/Metrics } & \multicolumn{2}{|c|}{$\begin{array}{c}\text { Device Specification } \\
\text { (Manufacturer Model and Specs) }\end{array}$} \\
\hline & $\begin{array}{l}\text { Monitoring } \\
\text { Period }\end{array}$ & $\begin{array}{l}\text { Training } \\
\text { Sessions }\end{array}$ & TS/Week & Sets & Match-Play & Internal Load & External Load & Internal Load & External Load \\
\hline $\begin{array}{l}\text { Baptista et al. } \\
\text { [97] }\end{array}$ & 11 weeks & 537 & $4 \mathrm{TS} / \mathrm{wk}$ & 630 & $15 \mathrm{M}$ & ND & $\begin{array}{l}\text { Distance and speed: TD } \\
\text { covered }(\mathrm{m}) \text {; HSR } \\
\text { peak } \\
\left(\mathrm{km} \times \mathrm{h}^{-1}\right) ; \text { and } \mathrm{SPR}_{\text {peak }} \\
\left(\mathrm{km} \times \mathrm{h}^{-1}\right) \\
\text { Acceleration: } \mathrm{ACC}_{\text {peak }} \\
\left(\mathrm{m} \times \mathrm{s}^{-2}\right) \text { and } \\
\mathrm{DEC}_{\text {peak }}\left(\mathrm{m} \times \mathrm{s}^{-2}\right)\end{array}$ & ND & $\begin{array}{c}\text { Stationary radio-based } \\
\text { tracking system (ZXY Sport } \\
\text { Tracking System, } \\
\text { Trondheim, Norway) }\end{array}$ \\
\hline Brito et al. [69] & 36 weeks & $2591 \mathrm{TS}$ & 5-11 TS/wk & ND & ND & $\begin{array}{l}\text { Perceived } \\
\text { Exertion: RPE, } \\
\text { sRPE, and } \\
\text { perceived } \\
\text { fatigue. }\end{array}$ & ND & $\begin{array}{l}\text { CR10 and fatigue } \\
\text { questionnaire. }\end{array}$ & ND \\
\hline $\begin{array}{c}\text { Campos- } \\
\text { Vazquez et al. } \\
\text { [50] }\end{array}$ & ND & ND & $\begin{array}{l}5 \mathrm{TS} / \mathrm{wk} \\
(\sim 90 \mathrm{~min})\end{array}$ & ND & ND & $\begin{array}{l}\text { HR: Edwards } \\
\text { TL and Stagno } \\
\text { TL/TRIMP } \\
\text { Perceived } \\
\text { Exertion: RPE } \\
\text { and sRPE. }\end{array}$ & ND & $\begin{array}{c}\text { CR10 and } 1 \mathrm{~Hz} \\
\text { short-range telemetry } \\
\text { system (Team 2, Polar } \\
\text { Electro, } \\
\text { Kempele, Finland). }\end{array}$ & ND \\
\hline $\begin{array}{l}\text { Casamichana } \\
\text { et al. [115] }\end{array}$ & ND & 44 TS & $\begin{array}{l}2 / 3 \mathrm{TS} / \mathrm{wk} \\
(\sim 90 \mathrm{~min})\end{array}$ & ND & ND & ND & $\begin{array}{c}\text { Distance and speed: TD } \\
\text { covered }(\mathrm{m}) \text {; DHS } \\
\left(\mathrm{km} \times \mathrm{h}^{-1}\right) ; \text { and DSS } \\
\left(\mathrm{km} \times \mathrm{h}^{-1}\right) \\
\text { Accelerometry: PL }(\mathrm{g}) \\
\text { Ratios/scores: WRR } \\
\left(\mathrm{km} \times \mathrm{h}^{-1}\right) \text {; FEHS } \\
\left(\mathrm{km} \times \mathrm{h}^{-1}\right) ; \text { and DHS } \\
\left(\mathrm{km} \times \mathrm{h}^{-1}\right)\end{array}$ & ND & $\begin{array}{c}10 \mathrm{~Hz} \text { GPS and } 100-\mathrm{Hz} \\
\text { MEMS (Catapult } \\
\text { MinimaxX Team Sport 4.0, } \\
\text { Melbourne, Australia). }\end{array}$ \\
\hline
\end{tabular}


Table 4. Cont.

\begin{tabular}{|c|c|c|c|c|c|c|c|c|c|}
\hline \multirow{2}{*}{$\begin{array}{l}\text { Reference } \\
\text { (Year) }\end{array}$} & \multicolumn{5}{|c|}{ Observations Sample } & \multicolumn{2}{|c|}{ Training Load Measures/Metrics } & \multicolumn{2}{|c|}{$\begin{array}{c}\text { Device Specification } \\
\text { (Manufacturer Model and Specs) }\end{array}$} \\
\hline & $\begin{array}{l}\text { Monitoring } \\
\text { Period }\end{array}$ & $\begin{array}{l}\text { Training } \\
\text { Sessions }\end{array}$ & TS/Week & Sets & Match-Play & Internal Load & External Load & Internal Load & External Load \\
\hline $\begin{array}{c}\text { Clemente et al. } \\
\text { [89] }\end{array}$ & ND & $44 \mathrm{TS}$ & $\begin{array}{l}3 \mathrm{TS} / \mathrm{wk} \\
(\sim 90 \mathrm{~min})\end{array}$ & ND & ND & ND & $\begin{array}{l}\text { Distance and speed: TD } \\
\text { covered }(\mathrm{m}) \text {; relative } \\
\text { distance covered or pace } \\
(\mathrm{m} / \mathrm{min}) \text {; } \mathrm{D} \text { in different } \\
\text { speed zones; maximum } \\
\text { speed }\left(\mathrm{km} \times \mathrm{h}^{-1}\right) \text {; and } \\
\text { number of sprints per } \\
\text { minute }\left(\mathrm{n} \times \min ^{-1}\right) \text {. } \\
\text { Accelerometry: } \mathrm{PL}(\mathrm{g}) \text {. }\end{array}$ & ND & $\begin{array}{c}10 \mathrm{~Hz} \text { GPS and 100-Hz } \\
\text { MEMS (JOHAN Sports, } \\
\text { Noordwijk, } \\
\text { The Netherlands). }\end{array}$ \\
\hline $\begin{array}{l}\text { Clemente et al. } \\
\text { [95] }\end{array}$ & 5 weeks & ND & $5 \mathrm{TS} / \mathrm{wk}$ & ND & ND & ND & $\begin{array}{l}\text { Distance and speed: TD } \\
\text { covered }(\mathrm{m}) \text {; relative } \\
\text { distance covered or pace } \\
(\mathrm{m} / \mathrm{min}) \text {; } \mathrm{D} \text { in different } \\
\text { speed zones; maximum } \\
\text { speed }\left(\mathrm{km} \times \mathrm{h}^{-1}\right) \text {; and } \\
\text { number of sprints per } \\
\text { minute }\left(\mathrm{n} \times \text { min }^{-1}\right) \text {. } \\
\text { Acceleration: ACC } \\
\left(\mathrm{m} \times \mathrm{s}^{-2}\right) \text { and } \\
\text { DEC }\left(\mathrm{m} \times \mathrm{s}^{-2}\right) \text {. } \\
\text { Accelerometry: PL }(\mathrm{g}) \text {. } \\
\text { Ratios/scores: TMr. }\end{array}$ & ND & $\begin{array}{c}10 \mathrm{~Hz} \text { GPS and } 100-\mathrm{Hz} \\
\text { MEMS (JOHAN Sports, } \\
\text { Noordwijk, } \\
\text { The Netherlands). }\end{array}$ \\
\hline $\begin{array}{l}\text { Clemente et al. } \\
\text { [88] }\end{array}$ & 7 weeks & ND & 5-6 TS/wk & ND & ND & ND & $\begin{array}{l}\text { Distance and speed: TD } \\
\text { covered }(\mathrm{m}) \text {; relative } \\
\text { distance covered or pace } \\
(\mathrm{m} / \mathrm{min}), \mathrm{D} \text { in different } \\
\text { speed zones; maximum } \\
\text { speed }\left(\mathrm{km} \times \mathrm{h}^{-1}\right) \text {; and } \\
\text { number of sprints per } \\
\text { minute }\left(\mathrm{n} \times \text { min }^{-1}\right) \text {. } \\
\text { Accelerometry: } \mathrm{PL}(\mathrm{g}) \text {. }\end{array}$ & ND & $\begin{array}{c}10 \mathrm{~Hz} \text { GPS and } 100-\mathrm{Hz} \\
\text { MEMS (JOHAN Sports, } \\
\text { Noordwijk, } \\
\text { The Netherlands). }\end{array}$ \\
\hline
\end{tabular}


Table 4. Cont.

\begin{tabular}{|c|c|c|c|c|c|c|c|c|c|}
\hline \multirow{2}{*}{$\begin{array}{l}\text { Reference } \\
\text { (Year) }\end{array}$} & \multicolumn{5}{|c|}{ Observations Sample } & \multicolumn{2}{|c|}{ Training Load Measures/Metrics } & \multicolumn{2}{|c|}{$\begin{array}{c}\text { Device Specification } \\
\text { (Manufacturer Model and Specs) }\end{array}$} \\
\hline & $\begin{array}{l}\text { Monitoring } \\
\text { Period }\end{array}$ & $\begin{array}{l}\text { Training } \\
\text { Sessions }\end{array}$ & TS/Week & Sets & Match-Play & Internal Load & External Load & Internal Load & External Load \\
\hline $\begin{array}{l}\text { Clemente et al. } \\
\text { [105] }\end{array}$ & 45 weeks & 197 TS & ND & ND & $44 \mathrm{MP}$ & ND & $\begin{array}{l}\text { Distance and speed: TD } \\
\text { covered }(\mathrm{m}) \text {; relative } \\
\text { distance covered or pace } \\
\left(\mathrm{m} \times \mathrm{min}^{-1}\right) \text {, D in different } \\
\text { speed zones; maximum } \\
\text { speed }\left(\mathrm{km} \times \mathrm{h}^{-1}\right) \text {; and } \\
\text { number of sprints per } \\
\text { minute }\left(\mathrm{n} \times \mathrm{min}^{-1}\right) \text {. }\end{array}$ & ND & $\begin{array}{c}\text { 18-Hz MEMS and 100-Hz } \\
\text { tri-axial accelerometer } \\
\text { (STATSports, Apex, Newry, } \\
\text { Northern Ireland). }\end{array}$ \\
\hline $\begin{array}{c}\text { Coutinho et al. } \\
{[47]}\end{array}$ & 22 weeks & ND & $3-4 \mathrm{TS} / \mathrm{wk}$ & ND & ND & HR: \%HRmax & $\begin{array}{l}\text { Distance and Speed: TD } \\
\text { covered }(\mathrm{m}) \text {; relative } \\
\text { distance or pace } \\
\left(\mathrm{m} \times \mathrm{min}^{-1}\right) \text {; } \mathrm{D} \text { in different } \\
\text { speed zone }\left(\mathrm{km} \times \mathrm{h}^{-1}\right) ; \\
\text { and sprints }(\text { number and } \\
\text { time interval). } \\
\text { Accelerometry: Absolute } \\
\text { and relative body } \\
\text { impacts }(\mathrm{g}) .\end{array}$ & $\begin{array}{l}5 \mathrm{~Hz} \text { short-range } \\
\text { telemetry system (Polar } \\
\text { Team System, Polar, } \\
\text { Kempele, Finland). }\end{array}$ & $\begin{array}{c}15 \text { Hz GPS and 100-Hz } \\
\text { MEMS (SPI-Pro X II, } \\
\text { GPSports, } \\
\text { Canberra, Australia) }\end{array}$ \\
\hline $\begin{array}{c}\text { Dalen et Lorås } \\
\text { [102] }\end{array}$ & 10 weeks & $38 \mathrm{TS}$ & $4 \mathrm{TS} / \mathrm{wk}$ & ND & $10 \mathrm{MP}$ & $\begin{array}{c}\text { HR: Banister } \\
\text { TL }\end{array}$ & $\begin{array}{l}\text { Distance and speed: TD } \\
\text { covered }(\mathrm{m}) \text {; relative } \\
\text { distance covered or pace } \\
\left(\mathrm{m} \times \mathrm{min}^{-1}\right) \text {; D in different } \\
\text { speed zones; and } \\
\text { maximum speed } \\
\left(\mathrm{km} \times \mathrm{h}^{-1}\right) \text {. } \\
\text { Acceleration: ACC } \\
\left(\mathrm{m} \times \mathrm{s}^{-2}\right) \text { and DEC } \\
\left(\mathrm{m} \times \mathrm{s}^{-2}\right)\end{array}$ & $\begin{array}{l}5 \mathrm{~Hz} \text { short-range } \\
\text { telemetry system (Polar } \\
\text { Team System, Polar, } \\
\text { Kempele, Finland) }\end{array}$ & $\begin{array}{l}10 \mathrm{~Hz} \text { and } 100-\mathrm{Hz} \text { MEMS } \\
\text { (Polar Team System, Polar, } \\
\text { Kempele, Finland). }\end{array}$ \\
\hline
\end{tabular}


Table 4. Cont

\begin{tabular}{|c|c|c|c|c|c|c|c|c|c|}
\hline \multirow{2}{*}{$\begin{array}{l}\text { Reference } \\
\text { (Year) }\end{array}$} & \multicolumn{5}{|c|}{ Observations Sample } & \multicolumn{2}{|c|}{ Training Load Measures/Metrics } & \multicolumn{2}{|c|}{$\begin{array}{c}\text { Device Specification } \\
\text { (Manufacturer Model and Specs) }\end{array}$} \\
\hline & $\begin{array}{l}\text { Monitoring } \\
\text { Period }\end{array}$ & $\begin{array}{l}\text { Training } \\
\text { Sessions }\end{array}$ & TS/Week & Sets & Match-Play & Internal Load & External Load & Internal Load & External Load \\
\hline $\begin{array}{l}\text { Gaudino et al. } \\
\text { [90] }\end{array}$ & 10 weeks & $628 \mathrm{TS}$ & $\begin{array}{c}24 \\
\text { TS/player }\end{array}$ & ND & ND & ND & $\begin{array}{l}\text { Distance and speed: } \mathrm{D} \text { in } \\
\text { different speed zone } \\
\left(\mathrm{km} \times \mathrm{h}^{-1}\right) \\
\text { Energy and metabolic } \\
\text { power: } \mathrm{P}_{\text {met }}\left(\mathrm{W} \times \mathrm{kg}^{-1}\right) \\
\text { and metabolic load } \\
\text { distance }\left(\mathrm{W} \times \mathrm{kg}^{-1}\right) \text {. }\end{array}$ & ND & $\begin{array}{c}15 \mathrm{~Hz} \text { GPS and } 100-\mathrm{Hz} \\
\text { MEMS } \\
\text { (SPI-Pro X II, GPSports, } \\
\text { Canberra, Australia). }\end{array}$ \\
\hline $\begin{array}{c}\text { Gaudino et al. } \\
\text { [118] }\end{array}$ & 38 weeks & 1892 TS & $\begin{array}{c}\text { 3-4 TS/wk } \\
\text { ( 60 min) }\end{array}$ & ND & ND & $\begin{array}{l}\text { Perceived } \\
\text { Exertion: RPE } \\
\text { and sRPE. }\end{array}$ & $\begin{array}{l}\text { Distance and speed: } \mathrm{D} \text { in } \\
\text { different speed zone } \\
\left(\mathrm{km} \times \mathrm{h}^{-1}\right) \text {. } \\
\text { Energy and metabolic } \\
\text { power: } \mathrm{P}_{\text {met }}\left(\mathrm{W} \times \mathrm{kg}^{-1}\right) \\
\text { and metabolic load } \\
\text { distance }\left(\mathrm{W} \times \mathrm{kg}^{-1}\right) \text {. } \\
\text { Accelerometry: } \\
\text { dynamic-stress load }(\mathrm{AU}) \text {. }\end{array}$ & CR10 & $\begin{array}{l}10 \mathrm{~Hz} \text { GPS and } 100-\mathrm{Hz} \\
\text { MEMS (Viper Pod, } \\
\text { STATSports, Newry, } \\
\text { Northern Ireland) }\end{array}$ \\
\hline $\begin{array}{l}\text { Impellizzeri } \\
\text { et al. [54] }\end{array}$ & 9 weeks & 479 TS & $\begin{array}{c}\text { 3-4 TS/wk } \\
\text { ( 60 min) }\end{array}$ & ND & ND & $\begin{array}{l}\text { HR: Edwards } \\
\text { TL, Banister } \\
\text { TL, and Lucia } \\
\text { TL. } \\
\text { Perceived } \\
\text { Exertion: RPE } \\
\text { and sRPE. }\end{array}$ & ND & $\begin{array}{c}\text { CR10 and } 5 \mathrm{~Hz} \\
\text { short-range telemetry } \\
\text { system (VantageNV, Polar } \\
\text { Electro, } \\
\text { Kempele, Finland). }\end{array}$ & ND \\
\hline Jeong et al. [55] & 10 weeks & $628 \mathrm{TS}$ & $\begin{array}{c}24 \\
\text { TS/players } \\
(\sim 60 \mathrm{~min})\end{array}$ & ND & $6 \mathrm{MP}$ & $\begin{array}{l}\text { HR: } \% \mathrm{HR}_{\max } \cdot \\
\text { Perceived } \\
\text { Exertion: RPE } \\
\text { and sRPE }\end{array}$ & ND & $\begin{array}{c}\text { CR10 and } 5 \mathrm{~Hz} \\
\text { short-range telemetry } \\
\text { system (Polar Team } \\
\text { System, Polar, } \\
\text { Kempele, Finland). }\end{array}$ & ND \\
\hline
\end{tabular}


Table 4. Cont.

\begin{tabular}{|c|c|c|c|c|c|c|c|c|c|}
\hline \multirow{2}{*}{$\begin{array}{l}\text { Reference } \\
\text { (Year) }\end{array}$} & \multicolumn{5}{|c|}{ Observations Sample } & \multicolumn{2}{|c|}{ Training Load Measures/Metrics } & \multicolumn{2}{|c|}{$\begin{array}{c}\text { Device Specification } \\
\text { (Manufacturer Model and Specs) }\end{array}$} \\
\hline & $\begin{array}{l}\text { Monitoring } \\
\text { Period }\end{array}$ & $\begin{array}{l}\text { Training } \\
\text { Sessions }\end{array}$ & TS/Week & Sets & Match-Play & Internal Load & External Load & Internal Load & External Load \\
\hline Kelly et al. [70] & 36 weeks & ND & $\begin{array}{c}\text { ND TS/wk } \\
(\sim 60 \mathrm{~min})\end{array}$ & ND & $49 \mathrm{MP}$ & $\begin{array}{c}\text { Perceived } \\
\text { Exertion: RPE } \\
\text { and sRPE }\end{array}$ & $\begin{array}{l}\text { Distance and speed: TD } \\
\text { covered }(\mathrm{m}) \text {; and } \mathrm{D} \text { in } \\
\text { different speed zones } \\
\quad\left(\mathrm{km} \times \mathrm{h}^{-1}\right) \text {. }\end{array}$ & CR 10 & $\begin{array}{c}10 \text { Hz GPS (SPI-Pro X II, } \\
\text { GPSports, Canberra, } \\
\text { Australia) and } \\
\text { semi-automatic } \\
\text { multiple-camera system } \\
\text { (Prozone Sports Ltd. }^{\circledR}, \\
\text { Leeds, United Kingdom) }\end{array}$ \\
\hline Kelly et al. [65] & 43 weeks & 1010 TS & $\begin{array}{c}55 \\
\text { TS/player }\end{array}$ & ND & ND & $\begin{array}{l}\text { HR: } \% \mathrm{HR}_{\max } \cdot \\
\text { Perceived } \\
\text { Exertion: RPE } \\
\text { and sRPE }\end{array}$ & ND & CR 10 & ND \\
\hline $\begin{array}{c}\text { Los Arcos et al. } \\
{[71]}\end{array}$ & 35 weeks & ND & $\begin{array}{c}4-5 \mathrm{TS} / \mathrm{wk} \\
(\sim 90-104 \\
\min )\end{array}$ & ND & ND & $\begin{array}{l}\text { Perceived } \\
\text { Exertion: } \\
\text { sRPEres-TL } \\
\text { and } \\
\text { sRPEmus-TL }\end{array}$ & ND & CR 10 & ND \\
\hline $\begin{array}{c}\text { Malone et al. } \\
\text { [56] }\end{array}$ & 7 weeks & $27 \mathrm{TS}$ & $3-4 \mathrm{TS} / \mathrm{wk}$ & ND & ND & $\begin{array}{l}\text { HR: } \% \mathrm{HR}_{\max } \\
\text { Perceived } \\
\text { Exertion: RPE } \\
\text { and sRPE }\end{array}$ & ND & $\begin{array}{c}\text { CR10 and Portable } \\
\text { team-based HR receiver } \\
{\text { (Acentas } \mathrm{GmBH}^{\circledR},} \\
\text { Freising, Germany; } \\
\text { Firstbeat Sports, } \\
\text { Jyväskylä, Finland) }\end{array}$ & $\begin{array}{c}15 \mathrm{~Hz} \text { GPS and } 100-\mathrm{Hz} \\
\text { MEMS } \\
\text { (SPI-Pro X II, GPSports, } \\
\text { Canberra, Australia) }\end{array}$ \\
\hline
\end{tabular}


Table 4. Cont.

\begin{tabular}{|c|c|c|c|c|c|c|c|c|c|}
\hline \multirow{2}{*}{$\begin{array}{l}\text { Reference } \\
\text { (Year) }\end{array}$} & \multicolumn{5}{|c|}{ Observations Sample } & \multicolumn{2}{|c|}{ Training Load Measures/Metrics } & \multicolumn{2}{|c|}{$\begin{array}{c}\text { Device Specification } \\
\text { (Manufacturer Model and Specs) }\end{array}$} \\
\hline & $\begin{array}{l}\text { Monitoring } \\
\text { Period }\end{array}$ & $\begin{array}{l}\text { Training } \\
\text { Sessions }\end{array}$ & TS/Week & Sets & Match-Play & Internal Load & External Load & Internal Load & External Load \\
\hline $\begin{array}{c}\text { Martin-Garcia } \\
\text { et al. [82] }\end{array}$ & 12 weeks & 17 TS & $\begin{array}{c}5 \mathrm{TS} / \mathrm{wk} \\
(\sim 83-92 \mathrm{~min})\end{array}$ & ND & ND & ND & $\begin{array}{l}\text { Distance and speed: TS } \\
\text { duration (min); TD covered } \\
(\mathrm{m}) \text {; and } \mathrm{D} \text { in different } \\
\text { speed zones }\left(\mathrm{km} \times \mathrm{h}^{-1}\right) \text {. } \\
\text { Acceleration: ACC } \\
\left(\mathrm{m} \times \mathrm{s}^{-2}\right) \text { and } \mathrm{DEC} \\
\left(\mathrm{m} \times \mathrm{s}^{-2}\right) \text {. } \\
\text { Metabolic power: AMP } \\
\text { power per second and kg } \\
\left(\mathrm{W} \times \mathrm{kg}^{-1}\right) \text {; and metabolic } \\
\text { load distance }\left(\mathrm{W} \times \mathrm{kg}^{-1}\right) \text {. }\end{array}$ & ND & $\begin{array}{c}10 \mathrm{~Hz} \text { GPS (Viper Pod, } \\
\text { STATSports, Canberra, } \\
\text { Australia) }\end{array}$ \\
\hline $\begin{array}{c}\text { Marynowicz } \\
\text { et al. [98] }\end{array}$ & 18 weeks & $\begin{array}{c}\text { 12-76 } \\
\text { TS/player }\end{array}$ & ND & 804 & ND & $\begin{array}{c}\text { Perceived } \\
\text { Exertion: RPE } \\
\text { and sRPE }\end{array}$ & $\begin{array}{c}\text { Distance and speed: TD } \\
\text { covered }(\mathrm{m}) \text {; relative } \\
\text { distance covered or pace } \\
\left(\mathrm{m} \times \mathrm{min}^{-1}\right) \text {; D in different } \\
\text { speed zones. } \\
\text { Acceleration: ACC } \\
\left(\mathrm{m} \times \mathrm{s}^{-2}\right) \text { and } \mathrm{DEC} \\
\left(\mathrm{m} \times \mathrm{s}^{-2}\right) \text {. } \\
\text { Accelerometry: } \mathrm{PL}(\mathrm{g}) \text {. }\end{array}$ & CR 10 & $\begin{array}{c}10 \mathrm{~Hz} \text { GPS and } 400 \mathrm{~Hz} \\
\text { tri-axial accelerometer } \\
\text { (Player Tek }{ }^{\mathrm{TM}} \text {, Catapult, } \\
\text { Melbourne, Australia). }\end{array}$ \\
\hline $\begin{array}{c}\text { Oliveira et al. } \\
\text { [72] }\end{array}$ & 45 weeks & $111 \mathrm{TS}$ & $4 \mathrm{TS} / \mathrm{wk}$ & ND & $\begin{array}{l}1 \mathrm{MP} / \mathrm{wk} \\
(90 \mathrm{~min})\end{array}$ & $\begin{array}{c}\text { Perceived } \\
\text { Exertion: RPE, } \\
\text { sRPE, and HI. }\end{array}$ & $\begin{array}{l}\text { Distance and speed: TS } \\
\text { duration }(\mathrm{min}) \text {; TD covered } \\
(\mathrm{m}) ; \mathrm{D} \text { in different speed } \\
\text { zones }\left(\mathrm{km} \times \mathrm{h}^{-1}\right) \text {; AvS } \\
\left(\mathrm{m} \times \mathrm{min}^{-1}\right) \\
\text { Acceleration: ACC } \\
\left(\mathrm{m} \times \mathrm{s}^{-2}\right) \text { and DEC } \\
\left(\mathrm{m} \times \mathrm{s}^{-2}\right) \\
\text { Accelerometry: PL }(\mathrm{g}) \text { and } \\
\text { number of impacts. }\end{array}$ & CR10 & $\begin{array}{l}10 \text { Hz GPS (Viper pod 2, } \\
\text { STATSports, Newry, } \\
\text { Northern Ireland) }\end{array}$ \\
\hline
\end{tabular}


Table 4. Cont

\begin{tabular}{|c|c|c|c|c|c|c|c|c|c|}
\hline \multirow{2}{*}{$\begin{array}{l}\text { Reference } \\
\text { (Year) }\end{array}$} & \multicolumn{5}{|c|}{ Observations Sample } & \multicolumn{2}{|c|}{ Training Load Measures/Metrics } & \multicolumn{2}{|c|}{$\begin{array}{c}\text { Device Specification } \\
\text { (Manufacturer Model and Specs) }\end{array}$} \\
\hline & $\begin{array}{l}\text { Monitoring } \\
\text { Period }\end{array}$ & $\begin{array}{l}\text { Training } \\
\text { Sessions }\end{array}$ & TS/Week & Sets & Match-Play & Internal Load & External Load & Internal Load & External Load \\
\hline $\begin{array}{l}\text { Owen et al. } \\
\text { [108] }\end{array}$ & 39 weeks & 2981 TS & 16-20 TS/M & ND & $\begin{array}{c}50 \\
\mathrm{MP} / \text { season }\end{array}$ & ND & $\begin{array}{l}\text { Distance and speed: TD } \\
\text { covered }(\mathrm{m}) \text { and } \mathrm{D} \text { in } \\
\text { different speed zones } \\
\left(\mathrm{km} \times \mathrm{h}^{-1}\right) \\
\text { Acceleration: ACC } \\
\left(\mathrm{m} \times \mathrm{s}^{-2}\right) \text { and DEC } \\
\left(\mathrm{m} \times \mathrm{s}^{-2}\right) \\
\text { Ratios/Scores: session } \\
\text { volume and intensity. }\end{array}$ & ND & $\begin{array}{l}\text { 10-Hz GPS (Viper, Statsport, } \\
\text { Newry, Northern Ireland) }\end{array}$ \\
\hline Owen et al. [99] & 42 weeks & 490 TS & $\begin{array}{c}5 \mathrm{TS} / \mathrm{wk} \\
(\sim 61-74 \mathrm{~min})\end{array}$ & ND & $37 \mathrm{MP}$ & $\begin{array}{l}\text { Perceived } \\
\text { Exertion: RPE, } \\
\text { CR10, and } \\
\text { SRPE }\end{array}$ & $\begin{array}{c}\text { Distance and speed: TD } \\
\text { covered }(\mathrm{m}) \text {; relative } \\
\text { distance covered or pace } \\
\left(\mathrm{m} \times \mathrm{min}^{-1}\right), \mathrm{D} \text { in different } \\
\text { speed zones }\left(\mathrm{km} \times \mathrm{h}^{-1}\right) \\
\text { maximum speed } \\
\left(\mathrm{km} \times \mathrm{h}^{-1}\right) \text {; and number of } \\
\text { sprints per minute } \\
\left(\mathrm{n} \times \mathrm{min}^{-1}\right)\end{array}$ & CR10 & $\begin{array}{l}10 \mathrm{~Hz} \text { GPS (Catapult } \\
\text { Innovations, } \\
\text { Melbourne, Australia). }\end{array}$ \\
\hline Rago et al. [107] & 6 weeks & 24 TS & $4 \mathrm{TS} / \mathrm{wk}$ & ND & ND & $\begin{array}{l}\text { Perceived } \\
\text { Exertion: RPE } \\
\text { and sRPE }\end{array}$ & $\begin{array}{c}\text { Distance and speed: TD } \\
\text { covered }(\mathrm{m}) ; \mathrm{D} \text { in different } \\
\text { speed zones }\left(\mathrm{km} \times \mathrm{h}^{-1}\right) ; \\
\text { and THIA }(\%) \text {. } \\
\text { Acceleration: ACC } \\
\left(\mathrm{m} \times \mathrm{s}^{-2}\right) \text { and DEC } \\
\left(\mathrm{m} \times \mathrm{s}^{-2}\right)\end{array}$ & CR 10 & $\begin{array}{c}\text { 10-Hz GPS (BT-Q1000 Ex, } \\
\text { QStarz, } \\
\text { Taipei, Taiwan) }\end{array}$ \\
\hline
\end{tabular}


Table 4. Cont.

\begin{tabular}{|c|c|c|c|c|c|c|c|c|c|}
\hline \multirow{2}{*}{$\begin{array}{l}\text { Reference } \\
\text { (Year) }\end{array}$} & \multicolumn{5}{|c|}{ Observations Sample } & \multicolumn{2}{|c|}{ Training Load Measures/Metrics } & \multicolumn{2}{|c|}{$\begin{array}{c}\text { Device Specification } \\
\text { (Manufacturer Model and Specs) }\end{array}$} \\
\hline & $\begin{array}{l}\text { Monitoring } \\
\text { Period }\end{array}$ & $\begin{array}{l}\text { Training } \\
\text { Sessions }\end{array}$ & TS/Week & Sets & Match-Play & Internal Load & External Load & Internal Load & External Load \\
\hline Rago et al. [61] & 13 weeks & $67 \mathrm{TS}$ & ND & 828 & $15 \mathrm{MP}$ & HR: \%HR $\max$ & $\begin{array}{l}\text { Distance and speed: TD } \\
\text { covered }(\mathrm{m}) \text { and } \mathrm{D} \text { in } \\
\text { different speed zones } \\
\left(\mathrm{km} \times \mathrm{h}^{-1}\right) \\
\text { Acceleration: ACC } \\
\left(\mathrm{m} \times \mathrm{s}^{-2}\right) \text { and DEC } \\
\left(\mathrm{m} \times \mathrm{s}^{-2}\right)\end{array}$ & $\begin{array}{c}5 \mathrm{~Hz} \text { short-range } \\
\text { telemetry system (WIMU } \\
\text { PRO; RealTrack Systems } \\
\text { SL, Almería, España). }\end{array}$ & $\begin{array}{l}\text { 10-Hz GPS with Triaxial } \\
\text { accelerometer (WIMU PRO; } \\
\text { RealTrack Systems SL, } \\
\text { Almería, España) }\end{array}$ \\
\hline $\begin{array}{c}\text { Sanchez- } \\
\text { Sanchez et al. } \\
{[91]}\end{array}$ & 8 weeks & $42 \mathrm{TS}$ & $\begin{array}{l}5 \mathrm{TS} / \mathrm{wk} \\
(\sim 75-120 \\
\min )\end{array}$ & ND & ND & ND & $\begin{array}{l}\text { Distance and speed: TS } \\
\text { duration (min); TD covered } \\
(\mathrm{m}) \text {; and } \mathrm{D} \text { in different } \\
\text { speed zones }\left(\mathrm{km} \times \mathrm{h}^{-1}\right) \\
\text { Acceleration: ACC } \\
\left(\mathrm{m} \times \mathrm{s}^{-2}\right) \text { and } \mathrm{DEC} \\
\left(\mathrm{m} \times \mathrm{s}^{-2}\right)\end{array}$ & ND & $\begin{array}{l}10 \text { Hz GPS (K-GPS, } \\
\text { Montelabbate, } \\
\text { Italy) }\end{array}$ \\
\hline $\begin{array}{c}\text { Swallow et al. } \\
{[110]}\end{array}$ & ND & 1029 TS & ND & ND & 3-55 MP & ND & $\begin{array}{c}\text { Distance and speed: TS } \\
\text { duration (min); TD covered } \\
(\mathrm{m}) \text {; and } \mathrm{D} \text { in different } \\
\text { speed zones }\left(\mathrm{km} \times \mathrm{h}^{-1}\right) \\
\text { Acceleration: ACC } \\
\left(\mathrm{m} \times \mathrm{s}^{-2}\right) \text { and DEC } \\
\left(\mathrm{m} \times \mathrm{s}^{-2}\right) \text {. } \\
\text { Accelerometry: PL }(\mathrm{g}) \text {. }\end{array}$ & ND & $\begin{array}{c}5 \mathrm{~Hz} \text { GPS and } 100 \mathrm{~Hz} \\
\text { tri-axial accelerometer } \\
\text { (Player Tek }{ }^{\mathrm{TM}} \text {, Catapult } \\
\text { Cloud, Catapult Sports } \\
\text { Group, } \\
\text { Australia). }\end{array}$ \\
\hline
\end{tabular}


Table 4. Cont.

\begin{tabular}{|c|c|c|c|c|c|c|c|c|c|}
\hline \multirow{2}{*}{$\begin{array}{l}\text { Reference } \\
\text { (Year) }\end{array}$} & \multicolumn{5}{|c|}{ Observations Sample } & \multicolumn{2}{|c|}{ Training Load Measures/Metrics } & \multicolumn{2}{|c|}{$\begin{array}{c}\text { Device Specification } \\
\text { (Manufacturer Model and Specs) }\end{array}$} \\
\hline & $\begin{array}{l}\text { Monitoring } \\
\text { Period }\end{array}$ & $\begin{array}{l}\text { Training } \\
\text { Sessions }\end{array}$ & TS/Week & Sets & Match-Play & Internal Load & External Load & Internal Load & External Load \\
\hline $\begin{array}{l}\text { Stevens et al. } \\
\text { [83] }\end{array}$ & 33 weeks & ND & $3 \mathrm{TS} / \mathrm{wk}$ & 536 & $1 / 2 \mathrm{MP} / \mathrm{wk}$ & HR: $\% \mathrm{HR}_{\max }$ & $\begin{array}{l}\text { Distance and speed: TD } \\
\text { covered }(\mathrm{m}) \text { and } \mathrm{D} \text { in } \\
\text { different speed zones } \\
\left(\mathrm{km} \times \mathrm{h}^{-1}\right) \text {. } \\
\text { Acceleration: ACC } \\
\left(\mathrm{m} \times \mathrm{s}^{-2}\right) \text {. } \\
\text { Accelerometry: PL }(\mathrm{g})\end{array}$ & $\begin{array}{l}\text { LPM-integrated Polar } \\
\text { Wearlink }^{\circledR} \text { technology } \\
\text { (Polar Electro Oy, } \\
\text { Kempele, Finland). }\end{array}$ & $\begin{array}{c}\text { LPM system (version } 05.91 \\
\text { T; Inmotiotec GmbH, } \\
\text { Regau, Austria). }\end{array}$ \\
\hline Vahia et al. [58] & $\sim 30$ weeks & 1029 TS & $4 \mathrm{TS} / \mathrm{wk}$ & ND & $3 \mathrm{MP}$ & $\begin{array}{l}\text { HR: Edwards } \\
\text { and Banister } \\
\text { TL. } \\
\text { Perceived } \\
\text { Exertion: RPE, } \\
\text { sRPE. }\end{array}$ & ND & $\begin{array}{c}\text { CR10 and } 1 \mathrm{~Hz} \\
\text { short-range telemetry } \\
\text { system (Team 2, Polar } \\
\text { Electro, Kempele, } \\
\text { Finland). }\end{array}$ & ND \\
\hline $\begin{array}{l}\text { Wrigley et al. } \\
\text { [60] }\end{array}$ & 30 weeks & $160 \mathrm{TS}$ & $7 \mathrm{TS} / \mathrm{wk}$ & 612 & $1 \mathrm{MP} / \mathrm{wk}$ & $\begin{array}{l}\text { HR: \%HR } \max \cdot \\
\text { Perceived } \\
\text { Exertion: RPE } \\
\text { and sRPE. }\end{array}$ & ND & $\begin{array}{c}\text { CR10 and } 1 \mathrm{~Hz} \\
\text { short-range telemetry } \\
\text { system (Team 2, Polar } \\
\text { Electro Oy, } \\
\text { Kempele, Finland). }\end{array}$ & ND \\
\hline $\begin{array}{l}\text { ACC-accelera } \\
\left(\geq 18 \mathrm{~km} \times \mathrm{h}^{-1}\right. \\
\text { positioning sys } \\
\text { ND-not descr } \\
\text { exertion; sRPE } \\
\text { TRIMP } \\
\text { MOD-n }\end{array}$ & $\begin{array}{l}\text { n; AMP_aver } \\
\text { SS-distance } \\
\text { s; HR-heart } r \\
\text { d; PL-player } \\
\text { sc-TL_-sessior } \\
\text { ified training i }\end{array}$ & $\begin{array}{l}\text { metabol } \\
\text { ered at sp } \\
\text { HR }_{\max } \\
\text { ad; Pmet- } \\
\text { atings of } \\
\text { ulse; TS- }\end{array}$ & $\begin{array}{l}\text { er; } \mathrm{AU}- \\
\text { eed }(\geq 21 \\
\text { lum heart } \\
\text { yalent me } \\
\text { lar traini }\end{array}$ & $\begin{array}{l}\text { Iry } \\
\mathrm{h}^{-1} \\
\text { Tzo } \\
\text { por } \\
\text {; sR }\end{array}$ & $\begin{array}{l}\text { AvS-averas } \\
\text { HS-frequen } \\
\text { actate thresh } \\
\text { Pmet-equi } \\
\text {-TL_-sessic }\end{array}$ & $\begin{array}{l}\text { eed; CR 10-Bo } \\
\text { efforts at high s1 } \\
\text { LPM-local posit } \\
\text { it metabolic pow } \\
\text { atings of respira }\end{array}$ & $\begin{array}{l}\text { Category-Ratio; D-distan } \\
\left(\geq 18 \mathrm{~km} \times \mathrm{h}^{-1}\right) ; \text { FESS—fr } \\
\text { neasurement; M-mesocycl } \\
\text { RPE-ratings of perceived } \\
\text { training load; TD—total di }\end{array}$ & $\begin{array}{l}\text { E-deceleration; DHS-di } \\
\text { cy of efforts at sprint speed } \\
\text { MS-micro-electrical mecha } \\
\text { on; SPR-sprinting; sRPE- } \\
\text {; THIA-total high-intensi }\end{array}$ & $\begin{array}{l}\text { nce covered at high speed } \\
\left.8 \mathrm{~km} \times \mathrm{h}^{-1}\right) \text {; GPS-global } \\
1 \mathrm{system} \text {; MP-match-play; } \\
\text { sions ratings of perceived } \\
\text { ictivity; TL-training load; }\end{array}$ \\
\hline
\end{tabular}




\subsection{Weekly Training Load Distribution Analysis}

Eleven reviewed articles analyzed weekly training load distribution. Two articles included only internal load measures, two articles evaluate only external load, and six studies analyzed both training load measures. Regarding periodization structure, seven studies analyzed weekly microcycle (1-game week), four studies quantified training load over mesocycles (week-block), and three articles included the training load quantification across different seasonal phases. One article did not specify the periodization structure for its analysis. Observations samples were collected from 27 to 2591 training sessions and varied from 4 to 6 training sessions per week. The monitoring period in the included studies ranged between 7 and 42 weeks. The included match-play ranged from 1 to 612 games.

The independent variables in the weekly training distribution analysis were age $(n=3)$, training day $(n=7)$, mesocycle structure $(n=3)$, training mode/type or sub-components $(n=1)$, playing position $(n=5)$, and contextual variables $(n=2)$. Table 5 provides the studies predominantly with a focus on weekly training load distribution analysis.

\subsection{Weekly Training Load and Match Load Distribution Analysis}

Fourteen articles analyzed the weekly training load distribution. Six articles assessed external load, two articles analyzed internal load measures, and two studies assessed both training load measures. Regarding the periodization structure, five studies evaluated the weekly microcycle (1-game week), two studies analyzed three different weekly microcycles (1-, 2- and 3-game week), and three studies quantified training load by mesocycles (weekblock). Any article included in this systematic review analyzed weekly training load and match load distribution across different seasonal phases. Observation samples were collected from 10 to 2981 training sessions and varied from 4 to 7 training sessions per week. The monitoring period in the included studies ranged from 3 and 55 weeks. The included match-play varied between 3 to 55 games.

The independent variables applied in weekly training load and match load distribution analysis were age of players $(n=1)$, training day $(n=2)$, weekly microcycle type $(n=3)$, mesocycle structure $(n=3)$, player's starting status (starters or non-starters) $(n=3)$, training mode/type or sub-components $(n=1)$, and playing position $(n=2)$. Table 6 provides the studies predominantly focusing on weekly training/match load distribution analysis.

\subsection{Relationships between Weekly Internal and External Load}

Eleven articles evaluated internal and external load relationships during training load quantification. Of these, five articles evaluated internal load relationships, five articles compared external load measures, and one study assessed the relationship between internal and external load. Four studies analyzed a weekly microcycle (1-game week) structure, and six articles did not specify the periodization structure. Observation samples were collected from 24 to 1029 training sessions and varied between 2 and 5 training sessions per week. The monitoring period in the included studies went from 9 to 43 weeks. The included match-play varied from 1 and 623 games.

All the eleven included articles in this sub-topic focused on comparison of internal and external load measures during training session. No other has analyzed the internal and external load relationships during match load. The independent variables applied in weekly training load distribution analysis were training day $(n=2)$, mesocycle structure $(n=1)$, training mode/type or sub-components $(n=2)$, playing position $(n=2)$, and training load indicators $(n=3)$. Table 7 provides the studies predominantly focusing on relationships between internal and external load during weekly training load. 
Table 5. Studies with predominantly focus on weekly training load distribution analysis.

\begin{tabular}{|c|c|c|c|c|c|}
\hline Reference (Year) & Study Purpose & Periodization Structure & Independent Variable & Main Findings & Practical Applications \\
\hline Abade et al. [47] & $\begin{array}{l}\text { Described time-motion and } \\
\text { physiological profile of } \\
\text { regular training sessions. }\end{array}$ & ND & Age of players & $\begin{array}{l}\text { Distance and speed: TD were higher in } \mathrm{U} 17(\mathrm{~F}= \\
45.84, p<0.001) \text {. High- and very-high intensity } \\
\text { activity were less in } \mathrm{U} 19(\mathrm{~F}=11.8, p>0.001) \text {. The } \\
\text { number of sprints performed were different } \\
\text { between } \mathrm{U} 17 \text { and } \mathrm{U} 19(\mathrm{~F}=-7.2, p<0.001) \\
\text { Accelerometry: Total and relative body impacts } \\
\text { were lower in } \mathrm{U} 15(\mathrm{~F}=7.3, p<0.01) \text {. } \\
\text { HR: HR values showed significant effects of zone } \\
(\mathrm{F}=575.7, p<0.001) \text { and interaction with age } \\
\text { group }(\mathrm{F}=-7.2, p<0.001) \text {. }\end{array}$ & $\begin{array}{l}\text { High variability between elite team TSs. } \\
\text { Constrained SSG to develop basic tactical } \\
\text { principles and technical skill may } \\
\text { promote low physio local demands. }\end{array}$ \\
\hline Akenhead et al. [53] & $\begin{array}{l}\text { Described the distribution of } \\
\text { external load during } \\
\text { in-season 1-game weeks in } \\
\text { in-season. Examined } \\
\text { inter-day and interposition } \\
\text { variation within microcycle } \\
\text { (focus on acceleration). }\end{array}$ & $\begin{array}{l}\text { Weekly microcycle (1-game } \\
\text { week) with "match day minus" } \\
\text { format: MD-5, MD-4, MD-3, } \\
\text { MD-2, MD-2, MD-1, MD. }\end{array}$ & $\begin{array}{l}\text { Training day and playing } \\
\text { position }\end{array}$ & $\begin{array}{l}\text { Distance and speed: Highest total weekly load } \\
\text { (\%) occurred on MD- } 4 \text {, with the lowest values on } \\
\text { MD- } 1 \text {. CM covered } \sim 8-16 \% \text { greater TD than other } \\
\text { playing positions (excluding WM) and covered } \\
\sim 17 \% \text { greater distance accelerating than CD }(p= \\
0.03, \mathrm{~d}=0.7) \text {. There are associations between AvS } \\
\left(\mathrm{m} \times \mathrm{s}^{-2}\right) \text { and the rate of accumulation for HSR, } \\
\text { SPD, }>1 \text { TOTAL, and }>3 \text { TOTAL. } \\
\text { Acceleration: ACC } / \text { DEC did not differ across } \\
\text { days with the greatest variation tending to be in } \\
\text { MD- } 1 . \text { No interaction between day and playing } \\
\text { positional were found. }\end{array}$ & $\begin{array}{l}\text { Monitoring only speed-based locomotor } \\
\text { variables may not provide sufficient } \\
\text { information about training demands. } \\
\text { Quantification acceleration variables may } \\
\text { add additional information. }\end{array}$ \\
\hline Brito et al. [69] & $\begin{array}{l}\text { Analyzed the influence of } \\
\text { match-related contextual } \\
\text { variables on TL and fatigue. } \\
\text { Concomitantly, investigated } \\
\text { if there were variations } \\
\text { throughout the season. }\end{array}$ & $\begin{array}{l}\text { Four different season phases: } \\
\text { preparation I ( } 3 \text { weeks), } \\
\text { competition I ( } 18 \text { weeks), } \\
\text { preparation II ( } 8 \text { weeks, winter } \\
\text { break) and competition II } \\
\text { (12 weeks). }\end{array}$ & $\begin{array}{l}\text { Contextual variables } \\
\text { (e.g., result of previous } \\
\text { MP, MP location, and } \\
\text { quality of opposition). }\end{array}$ & $\begin{array}{l}\text { Distance and speed: Weekly TLs were higher } \\
\text { after playing a defeat or draw ( } p \leq 0.05 ; \\
\mathrm{d}=0.30-0.45) \text { and after an away MP }(p \leq 0.05 ; \\
\mathrm{d}=0.23) \text {. Weekly TL decreased as the season } \\
\text { progressed ( } p<0.001) \text {. } \\
\text { Perceived Exertion: Internal load variation } \\
\text { ranged } 5 \text { to } 72 \% \text { throughout the season }(29-49 \% \text { to } \\
\text { weekly TL; } 18-44 \% \text { to fatigue scores). }\end{array}$ & $\begin{array}{l}\text { Internal load variability within a season } \\
\text { may need a more individualized } \\
\text { approach to prepare initial and } \\
\text { subsequent match conditions. Adding } \\
\text { that variability together relatively stable } \\
\text { fatigue scores may modulate pace } \\
\text { during training. }\end{array}$ \\
\hline Clemente et al. [89] & $\begin{array}{l}\text { Analyzed intra-week } \\
\text { variations during a typical } \\
\text { weekly external load and } \\
\text { compared variance in four } \\
\text { professional teams. }\end{array}$ & $\begin{array}{l}\text { Weekly microcycle (1-game week) } \\
\text { with “match day minus" format: } \\
\text { MD+1, MD + 2, MD-5, MD-4, } \\
\text { MD-3, MD-2, MD-2, MD-1, MD. }\end{array}$ & Training day & $\begin{array}{l}\text { Distance and speed: MD- } 1 \text { had significantly less } \\
\text { training while other days were more intense } \\
(p=0.001) \text {. Portuguese team showing } \\
\text { significantly higher intensity (SPR distance) and } \\
\text { volume (total distance) in all days with exception } \\
\text { of MD- } 1 \text { than the Dutch team }(p<0.05) \text {. } \\
\text { Accelerometry: Dutch team had significantly } \\
\text { greater value of PL in MD-3 }(p=0.005 ; \mathrm{d}=1.18) \\
\text { and Portuguese team had higher PL in the MD+2 } \\
(p=0.005 ; \mathrm{d}=1.78) \text {. }\end{array}$ & $\begin{array}{l}\text { The training TL and tapering strategies } \\
\text { were different between teams in different } \\
\text { countries. However, both teams applied a } \\
\text { significant tapering phase in the last two } \\
\text { days before the competition in an attempt } \\
\text { to reduce residual fatigue accumulation. }\end{array}$ \\
\hline
\end{tabular}


Table 5. Cont.

Reference (Year) $\quad$ Study Purpose $\quad$ Periodization Structure Independent Varible

Quantified weekly external compared variance in two

Described the time-motion and physiological

Coutinho et al. [47] erformance profiles during a typical weekly microcycle. load and intra-week variations during a pre-season training and professional teams.

Weekly microcycle (1-game week) with "match day minus" format: MD-5, MD-4, MD-3, MD-2, MD-2, MD-1, MD.

Training day
Weekly microcycle (1-game week) divided into: post-matc (session after the match), pre-match (session before the match), and middle week

(average of remaining sessions).
Age of players and weekly microcycle division (pre-match, mid-week and post-match).
Season phases divided into

preseason and in-season.

Quantified and compared
during a preseason and in-season training process. raining mode subdivided.

echnicaltractining, pal/tactical training, and physical and
Training mode/type or sub-components and season phase.

technical/tactical training.

\section{Main Findings}

Practical Applications

\section{Distance and speed: Weekly TL presented}

significant differences between TS considering the duration $(p=0.011)$, walking distance $(p=0.017)$, running distance $(p=0.004)$, and number of sprints $(p=0.006)$. Variations between weeks were competition and monitoring weekly small and intra-week variations in the measures associated with great volume and lower intensity. Accelerometry: Weekly TL also presented significant differences between TS considering PL $(p=0.040)$.

\section{Distance and Speed:}

U15 Mid-week showed a higher number of sprints, distance covered in intermediate speed zones, and time spent above $90 \% \mathrm{HR}_{\max }$

Pre-match presented a higher distance covered

above $18 \mathrm{~km} \times \mathrm{h}^{-1}$ and time spent below 75\% HR $\max$.

U17 Pre-match and post-match presented lowe distance covered values than mid-week.

U19 Post-match showed higher distance covere above $13 \mathrm{~km} \times \mathrm{h}^{-1}$, body impacts $(>10 \mathrm{~g})$, and time spent above $85 \% \mathrm{HR}_{\max }$.

Accelerometry:

U15 body impacts showed significant differences across all TSs.

U17 pre-match and post-match presented moderate differences in body impacts.

U19 middle-week showed higher values in body impacts and pre-match presented less values than the middle-week ( $35 \%$ to $100 \%$ ).

HR and Perceived Exertion: Preseason load was higher than in-season load $(p<0.05)$. Time spent in $80-100 \%$ maximum heart rate zones greater proportion in preseason and in-season, while technical/tactical sessions had higher intensities in the pre-season $(p<0.05)$.
Appropriate physical and physiological oad during middle-week TSs should be ensured. Understanding the weekly training and match load variations can contribute to optimizing short- and mid-term planning during different developmental stages.
Preseason is more intense than in-season training. Emphasis on higher intensities and time spent in technical/tactical specific TSs may provide the necessary physiological conditioning. 
Table 5. Cont.

Reference (Year) $\quad$ Study Purpose $\quad$ Periodization Structure Independent Variable

\section{Season phases divided into}

preseason and in-season.

Quantified the seasonal TL,
$\begin{gathered}\text { Mesocycle ranged from } 1 \text { to } 6 \\ \text { weeks (week blocks) and weekly }\end{gathered}$ weeks (week blocks) and weekly "match day minus" format:

MD-5, MD-4, MD-3, MD-2, MD-2, MD-1, MD and in-season phase.
Independent Variable

Season phase, mesocycle, training day and playing position.
Quantified TL using s-RPE and $\mathrm{HI}$ across mesocycle during an in-season

Oliveira et al. [72]
Mesocycle (one month) and weekly microcycle (1-game week) with "Match day minus" format: MD-5, MD-4, MD-3, MD-2, MD-2,

$$
\text { MD-1, MD. }
$$

Mesocycle, training day, and playing position
Analyzed a training mesocycle whilst quantifyin

Owen et al. [108] and examinat math location, match and age of players.
Mesocycle $(6 \times 1$-week block $)$ and weekly microcycle (1-game week) with "match day minus" format: MD-4, MD-3, MD-2,

$$
\text { MD-2, MD-1, MD. }
$$

Mesocycle, training day, contextual variables (match location and match status), age of players, and playing position.

\section{Main Findings}

Practical Application

HR and Perceived Exertion: typical daily TL did not differ during each week of the preseason.

Daily TD covered was greater in the 1st mesocycle than in the 6 th. $\% \mathrm{HR}_{\max }$ values were also greater in the 3rd mesocycle than in the 1st. TL was lowe on MD-1 (regardless mesocycle) and no differences were found in other days (MD-2 to MD-5). Positional differences were found during both preseason and in-season phases. In total, CM and WD covered the highest TD. Defenders (CD and $\mathrm{WD}$ ) displayed higher $\% \mathrm{HR}_{\max }$ values.

Distance and speed: Daily TD covered was higher at the start (M1 and M3) compared to the final mesocycle (M10) of season. HSR distance was greater in M1 than M5. CM covered more distance and WM cover more distance at HSR. Acceleration and accelerometry: All TL variables expressed significant lower values to other days prior to a MP and no difference between player positions $(p<0.01)$

erceived Exertion: Perceptual response was higher in M1 in comparison to the last mesocyc sRPE presented a non-perfect pattern by decreasing values until MD-1: MD-5 $<$ MD-4 $<$ MD-3 > MD-2 > MD-1. HI showed minor variations across mesocycles and in days before MP.

Distance and speed: Typical daily TL did not differ throughout each week of the mesocycle in-season period TL were significantly lower on in-seas ( $<0.05)$. Tow MD-1 $(p<0.05)$. Lower AvSs were reported in training post-successful MP compared to defeats $(p<0.05)$, and more specifically when a MP was played away compared to home fixtures $(p<0.05)$. Acceleration and accelerometry: Significant differences in physical outputs were also found between MD-2, MD-3 and MD-4 $(p<0.05)$.
Quantify TL using different measures can provide physiological patterns across a full competitive season. First and last TS optimized recovery and prevent fatigue accumulation. Positional differences should also be considered in the loading analysis.
Analysis of training mesocycle and microcycle positional demands may provide useful information to training program design and tactical strategy. Physical outputs on MD-2, MD-3, and MD-4 highlighting a structured

periodized tapered approach.
Combination of different TL measures the patterve ovidence to fully evaluate MD-1 press ore across the in-season. load (regardless of mesocycle) and HI did not change, except for MD+1. 
Table 5. Cont

\begin{tabular}{|c|c|c|c|c|c|}
\hline Reference (Year) & Study Purpose & Periodization Structure & Independent Variable & Main Findings & Practical Applications \\
\hline Rago et al. [61] & $\begin{array}{l}\text { Quantified the weekly TL } \\
\text { according to different } \\
\text { match-related } \\
\text { contextual factors. }\end{array}$ & $\begin{array}{l}\text { Training structure included } \\
\text { speed endurance training (e.g., } \\
\text { repeated sprint activity) and } \\
\text { aerobic high-intensity training } \\
\text { (e.g., interval training). The } \\
\text { remaining TS mainly concerned } \\
\text { ball-possession games and } \\
\text { team/opponent tactics. } \\
\text { Individual/reconditioning } \\
\text { sessions were excluded from the } \\
\text { analysis. The periodization } \\
\text { structure has not been described. }\end{array}$ & $\begin{array}{l}\text { Contextual variables } \\
\text { (opponent standard, } \\
\text { match location, and } \\
\text { match outcome). }\end{array}$ & $\begin{array}{l}\text { Distance and speed: TD covered and HSR during } \\
\text { training were higher in the week after playing } \\
\text { against a bottom-level or top-level opponent } \\
\text { compared to a medium-level opponent }(p<0.05) \text {. } \\
\text { TD covered and HSR was higher in the week } \\
\text { following a draw or a win, and higher before a } \\
\text { loss compared to a draw }(p<0.05) \text {. } \\
\text { Acceleration: The decrease in training volume } \\
\text { (e.g., TD) and mechanical work (accelerations and } \\
\text { decelerations) performed throughout the season } \\
\text { may have been related to changes in training } \\
\text { activities prescribed by the technical staff as a } \\
\text { consequence of cumulative seasonal TL }(p<0.05) \text {. }\end{array}$ & $\begin{array}{l}\text { Weekly TL seems to be slightly affected } \\
\text { by match-related contextual variables, } \\
\text { with special emphasis on the opponent } \\
\text { standard and match outcome. Higher } \\
\text { training volume was observed before and } \\
\text { after playing against a top-level opponent, } \\
\text { and after losing a match, whereas the } \\
\text { volume of high-intensity training seems } \\
\text { to be higher when preparing for a game } \\
\text { against a top-level opponent. }\end{array}$ \\
\hline \multicolumn{6}{|c|}{ 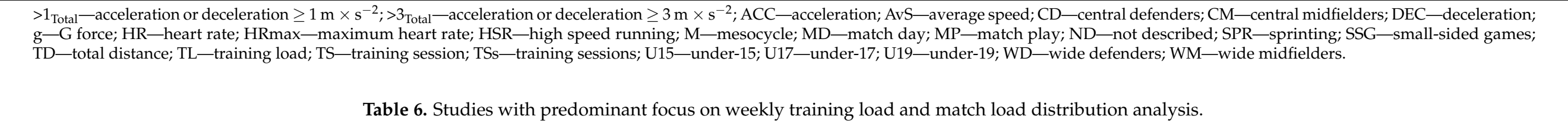 } \\
\hline Reference (Year) & Study Purpose & Periodization Structure & Independent Variable & Main Findings & Practical Applications \\
\hline Anderson et al. [106] & $\begin{array}{l}\text { Quantified training load and } \\
\text { match load during a season } \\
\text { within starting status (starters, } \\
\text { non-starters, and fringe). }\end{array}$ & $\begin{array}{l}\text { Mesocycle (5 different } \\
\text { in-season periods): } \\
4 \times 8 \text {-weeks (periods 1-4) and } \\
1 \times 7 \text {-weeks (period 5). }\end{array}$ & $\begin{array}{l}\text { Player's starting status } \\
\text { (starters, non-starters, } \\
\quad \text { or fringe) }\end{array}$ & $\begin{array}{l}\text { Distance and speed: Starters completed more } \\
\text { moderate intensity running, HSR, and SPR than } \\
\text { non-states }(p<0.01) \text {. Starters also completed more } \\
\text { SPR than fringe players }(p<0.01) \text {. }\end{array}$ & $\begin{array}{l}\text { Seasonal volume and intensity training } \\
\text { are dependent on player's match starting } \\
\text { status and must be considered for } \\
\text { training program design. }\end{array}$ \\
\hline
\end{tabular}


Table 6. Cont.

\begin{tabular}{|c|c|c|c|c|c|}
\hline Reference (Year) & Study Purpose & Periodization Structure & Independent Variable & Main Findings & Practical Applications \\
\hline Baptista et al. [97] & $\begin{array}{l}\text { Quantified the most } \\
\text { demanding passages of play in } \\
\text { training sessions and matches } \\
\text { (5-min peaks); and evaluated } \\
\text { the accumulated load of typical } \\
\text { microcycles and official } \\
\text { matches, according to } \\
\text { playing position. }\end{array}$ & $\begin{array}{c}\text { Weekly microcycle (1-game } \\
\text { week) with “match day minus” } \\
\text { format: MD+1C, MD+1R, } \\
\text { MD-4, MD-3, MD-2, MD-2, } \\
\text { MD-1, MD }\end{array}$ & $\begin{array}{l}\text { Playing position and } \\
\text { weekly microcycle. }\end{array}$ & $\begin{array}{l}\text { Distance and speed: Training values for SPR and } \\
\text { HSR distance were lower (36-61\% and } 57-71 \% \text { ) } \\
\text { than for acceleration variables. The highest } \\
\text { difference was verified on the 5-min peaks for } \\
\text { SPR } \\
\text { peak, with FB achieving during the microcycle } \\
\text { only } 64 \% \text {, while CB, CM, and FW levelled and } \\
\text { overperformed the match values (107\%, } 100 \% \text {, } \\
\text { and } 107 \% \text {, respectively). } \\
\text { Acceleration: Correlations match demands were } \\
\text { overperformed for ACC counts (131- } 166 \% \text { ) and } \\
\text { DEC counts (108- } 134 \%) \text {, according all position. } \\
\text { Training values were higher than SPR and } \\
\text { HSR distance. }\end{array}$ & $\begin{array}{l}\text { Differences observed across playing } \\
\text { positions in matches and microcycles } \\
\text { underline the lack of position specificity } \\
\text { of common training drills/sessions. } \\
\text { Coaches and practitioners must keep in } \\
\text { mind that the absolute TL accumulated } \\
\text { by players of different positions, so } \\
\text { analyzing the relative TL (according to } \\
\text { the match demands) may be a much } \\
\text { better and more valuable way of } \\
\text { managing and evaluating the } \\
\text { players periodization. }\end{array}$ \\
\hline Dalen et Lorås [102] & $\begin{array}{l}\text { Analyzed physical (locomotor } \\
\text { activities) and physiological } \\
\text { (Banister's training impulse) } \\
\text { in-season training load } \\
\text { between starters } \\
\text { and substitutes. }\end{array}$ & ND & $\begin{array}{l}\text { Player's starting status } \\
\text { (starters and } \\
\text { non-starters) }\end{array}$ & $\begin{array}{l}\text { Distance and speed: Starting players } \\
\text { demonstrated significantly higher average weekly } \\
\text { physical load compared to the non-starters for all } \\
\text { variables: TD, running, HSR, and SPR }(p<0.001) \text {, } \\
\text { number of ACC and SPR }(p<0.001) \text {. Similarly, } \\
\text { Banister's TL ( } p<0.001) \text { was significantly higher } \\
\text { within week than starters than non-starters. }\end{array}$ & $\begin{array}{l}\text { The weekly accumulated high-speed } \\
\text { running and sprint distances were largely } \\
\text { related to match playing time. Therefore, } \\
\text { weekly fitness-related adaptations in } \\
\text { running at high speeds seem to favor the } \\
\text { starters in a soccer team. }\end{array}$ \\
\hline Clemente et al. [95] & $\begin{array}{l}\text { Described the training/match } \\
\text { ratios and variations between } \\
\text { different weekly microcycle } \\
\text { type. Investigated relationship } \\
\text { within weekly accumulated TL } \\
\text { and match load. }\end{array}$ & $\begin{array}{c}\text { Three different weekly } \\
\text { microcycle: week with } 5 \text { TSs }(5 \\
\text { dW), } 4 \text { TSs }(4 \mathrm{dW}) \text { or } 3 \text { TSs } \\
(3 \mathrm{dW})\end{array}$ & Weekly microcycle type & $\begin{array}{l}\text { Distance and speed: TDr, HSRr, and SPRr were } \\
\text { significantly greater in } 5 \mathrm{dW}(p<0.001) \text {. } \\
\text { Acceleration and accelerometry: Correlations } \\
\text { between the weekly TL and the match load of the } \\
\text { same week were small for PL }(\mathrm{r}=0.25(0.13 ; 0.36)) \text {, } \\
\text { ACC }(\mathrm{r}=0.29(0.17 ; 0.40)) \text { and DEC }(\mathrm{r}=0.23 \\
(0.11 ; 0.35)) .\end{array}$ & $\begin{array}{l}\text { Additional TSs, it may be necessary to } \\
\text { promote differences between weekly } \\
\text { accumulated TL and the load imposed in } \\
\text { a single MP. Relationship between weekly } \\
\text { accumulated TL and weekly MP are } \\
\text { dynamic and unpredictable which may } \\
\text { be impossible for accumulated weekly TL } \\
\text { and their variations to be adjusted } \\
\text { according to match loads. }\end{array}$ \\
\hline Clemente et al. [105] & $\begin{array}{l}\text { Analyzed the variations of } \\
\text { acute load, training monotony, } \\
\text { and training strain among } \\
\text { pre-season, mid-season and } \\
\text { end-season according } \\
\text { playing position. }\end{array}$ & $\begin{array}{l}\text { Mesocycle ( } 5 \text { different } \\
\text { in-season periods): (i) } \\
\text { pre-season (week } 1 \text { to week } 6 \text { ); } \\
\text { mid-season or first half of the } \\
\text { season (week } 6 \text { to week } 33 \text { ); } \\
\text { and end-season or second half } \\
\text { of the season (week } 34 \text { to } \\
\text { week 45). }\end{array}$ & Season phase & $\begin{array}{l}\text { Distance and speed: Training monotony and } \\
\text { training strain for HSR were meaningfully greater } \\
\text { in pre-season than in the mid-season and greater } \\
\text { than the end-season }(p<0.001) \text {. The training } \\
\text { monotony for the sprints was meaningfully } \\
\text { greater in pre-season than in the mid-season and } \\
\text { greater than the end-season }(p<0.001) \text {. } \\
\text { Comparisons between playing positions revealed } \\
\text { small-to-moderate effect size differences mainly } \\
\text { for the number of sprints in acute load, training } \\
\text { monotony, and training strain. }\end{array}$ & $\begin{array}{l}\text { Acute load, training monotony, and } \\
\text { training strain occurred in the pre-season } \\
\text { and progressively decreased across the } \\
\text { season. Moreover, external defenders and } \\
\text { wingers were subjected to meaningfully } \\
\text { greater acute load and training strain for } \\
\text { HSR and number sprints during the } \\
\text { season compared to the } \\
\text { remaining positions. }\end{array}$ \\
\hline
\end{tabular}


Table 6. Cont.

$\begin{array}{llll}\text { Reference (Year) } & \text { Study Purpose } & \text { Periodization Structure } & \text { Independent Variable }\end{array}$

Analyzed TL and match load across a full season.
Mesocycle ranged from 6 to 9 weeks. playing position respiratory and muscular perceived $\mathrm{TL}$ accumulation depending on the player participation
Quantified and compared the
Mesocycle ranged from 6 to 8 weeks (week blocks) and weekly microcycle (1-game week) with "match day minus" format: MD-5, MD-4 MD-3, MD-2, MD-2, MD-1, MD.
Player's starting status (starters or non-starters) mesocycle and training day.

\section{Main Findings}

\section{Distance and speed: Daily TDs were higher}

during the early stages (M1 and M2) of the competition period. Overall, high-speed activity was similar between mesocycles. Weekly TL was greater on MD and lower MD-1 $(p<0.001)$. TD progressively decreased over the MD-3 $(p<0.001)$. High-speed distance was greater MD-3 while very high-speed distance was greater on MD-3 and MD-2 than MD-1 $(p<0.001)$

Perceived Exertion: Daily sRPE was also higher across early season stages. sRPE progressively decreased over the MD-3 $(p<0.001)$ as well as TD $(p<0.001)$.

Perceived Exertion: Weekly TL variation across the in-season blocks was trivial-small (except between block 2-block3). Substantial TL

differences were found between training days. Perceived TL across the season displayed

Weekly TL was a progressive increase up to MD-3 applied to $72 \mathrm{~h}$ before the MD to followed by a decrease until MD-1. sRPEres/sRPEmus-TL reported during MD was very similar between starters and non-starters.

Distance and speed: TL declined as competition approached (MD-4 $>$ MD-3 $>$ MD-2 $>$ MD-1; $p<0.05)$. MD $+1 \mathrm{C}$ by players with game time was higher than $\mathrm{MD}+1 \mathrm{R}(p<0.05)$. FB performed more high-speed running and SPR than other positions at MD-3 and MD-4 ( $p<0.05 ;(0.8-1.7))$. Weekly TL variation was $\sim 40 \%$ for MD-3 and
Determined the external load across playing position and relative for a structured

Martin-Garcia et al [82] variation the day after competition for players with or without MP time.
Weekly microcycle (1-game week) with "match day minus"

format: $\mathrm{MD}+1 \mathrm{C}, \mathrm{MD}+1 \mathrm{R}$, MD-4, MD-3, MD-2, MD-2, MD- 1, MD.
Playing position and training day.
$\mathrm{MD}-4$ to $\sim 80 \%$ for MD+1R.

Acceleration: ACC/DEC represented $50 \%$ of that performed in competition

for MD+1C (80-86\%), MD-4 (71-72\%), MD-3

Metabolic power: $\mathrm{MD}+1 \mathrm{C}$ demonstrated greater HLMD and AMP than MD+1R $(p<0.05 ;(1.4-1.6))$. TL declined as competition approached (MD-4 to MD-1) as well as HMLD and AMP ( $p<0.05$; ES: 1.5-3.0).
(62-69\%), and MD-2 (56-61\%).
Limited TL variation across mesocycles suggests that training schedule reflecting the nature of the competition demands. TL periodization included a three-day period leading into competition. employed a highly repetitive likely

progressively between MD-3 and MD.

(n)


Table 6. Cont.

\begin{tabular}{|c|c|c|c|c|c|}
\hline Reference (Year) & Study Purpose & Periodization Structure & Independent Variable & Main Findings & Practical Applications \\
\hline Owen et al. [99] & $\begin{array}{l}\text { Investigated multi-metric } \\
\text { monitoring method } \\
\text { highlighting TL and its } \\
\text { relationship to MP. }\end{array}$ & $\begin{array}{l}\text { Weekly microcycle (1-game } \\
\text { week) with "match day minus" } \\
\text { format: MD-5, MD-4, MD-3, } \\
\text { MD-2, MD-2, MD-1, MD. }\end{array}$ & Training day & $\begin{array}{l}\text { Distance and speed: Significant differences } \\
\text { between daily TL and competitive TL. } \\
\text { Additionally, significant differences between } \\
\text { training days for both volume- and intensity } \\
\text { session scores }(p<0.05) \text {. No differences were } \\
\text { found between MD- } 1 \text { and MD-2 session scores. }\end{array}$ & $\begin{array}{l}\text { Specific multi-modal approach may } \\
\text { combine key mechanical volume and } \\
\text { intensity metrics to player monitoring } \\
\text { strategies and tapering approaches. The } \\
\text { TL and match load relationships could } \\
\text { provide a better understanding to the } \\
\text { need for prepare players individually in } \\
\text { line with MP demands. }\end{array}$ \\
\hline $\begin{array}{l}\text { Sanchez-Sanchez } \\
\text { et al. [91] }\end{array}$ & $\begin{array}{l}\text { Quantified the external load } \\
\text { during in-season training } \\
\text { microcycles and examined its } \\
\text { relationship to the } \\
\text { competition demands. }\end{array}$ & $\begin{array}{l}\text { Weekly microcycle (1-game } \\
\text { week) with "match day minus" } \\
\text { format: MD-5, MD-4, MD-3, } \\
\text { MD-2, MD-2, MD-1, MD. }\end{array}$ & Training day & $\begin{array}{l}\text { Distance and speed: External load variables were } \\
\text { similar between the four microcycles. MD-2 } \\
\text { presented highest TL on time, TD, HSR and SPR } \\
\text { compared MD+1, MD-3 and MD- }(p<0.01) \text {. } \\
\text { Acceleration: Aside from training duration, all } \\
\text { external loads variables were lower during } \\
\text { training sessions compared to official matches } \\
(p<0.05) \text {. }\end{array}$ & $\begin{array}{l}\text { Absolute and relative external load values } \\
\text { allow to more accurately know the load } \\
\text { applied. MP constitutes the highest load } \\
\text { during a typical competitive microcycle } \\
\text { and MD-2 contain the weekly peak load. }\end{array}$ \\
\hline Swallow et al. [110] & $\begin{array}{l}\text { Quantified the external TL } \\
\text { across both training and } \\
\text { competitive matches during } \\
\text { the season. Examined the } \\
\text { influence of one and two match } \\
\text { weekly microcycles on the } \\
\text { external TL. }\end{array}$ & $\begin{array}{l}\text { Weekly microcycle (1-game } \\
\text { week) with "match day minus" } \\
\text { format: MD-5, MD-4, MD-3, } \\
\text { MD-2, MD-2, MD-1, MD. }\end{array}$ & & $\begin{array}{l}\text { Distance and speed: TD and HSR were higher on } \\
\text { MD and MD-5. MD-4 displayed significantly } \\
\text { higher values compared to MD-1 and MD-2. The } \\
\text { 2-game week presented a TD higher on MD-1 } \\
\text { when compared to 1-game week. However, lower } \\
\text { values were observed for duration and HSR on } \\
\text { MD-2 and MD-4 during the 2-game week } \\
\text { compared to the 1-game week. } \\
\text { Acceleration: Higher values recorded on MD for } \\
\text { number of ACC. ACC data were influenced for } \\
\text { the different game week schedule. } \\
\text { Accelerometry: PL was also higher on MD and } \\
\text { MD-5. The 2-game week presented a higher PL on } \\
\text { MD-1 than 1-game week. }\end{array}$ & $\begin{array}{l}\text { Progressive reduction in TD, PL, HSR, } \\
\text { and ACC leading into competitive } \\
\text { matches based on MD- analysis. } \\
\text { However, some variability exists in TL } \\
\text { prescription as a result of different } \\
\text { 1-game week schedules (i.e., 1-game week } \\
\text { vs. 2-game week). }\end{array}$ \\
\hline
\end{tabular}


Table 6. Cont.

$\begin{array}{llll}\text { Reference (Year) } & \text { Study Purpose } & \text { Periodization Structure } & \text { Independent Variable }\end{array}$

Quantified and compared the

$\mathrm{L}$ of training days and MP. Compared training of

Stevens et al. [80] nonstarters the day after the match with regular training of starters and non-starters.
Weekly microcycle (1-game week) with "match day minus" format: MD-5, MD-4, MD-3,

MD-2, MD-2, MD-1, MD (starters or non-starters)

(n)

\section{Main Findings}

Distance and speed: TL was lower when training approached MD. Training values for running and HSR were lower than for TD, and all considerably lower than MD values. Non-starters training was lower loading than regular training for almost all variables on MD-4 and several high-intensity variables on MD-3 and MD.

Acceleration and metabolic power: Medium and high accelerations and decelerations during training were more similar to match values. MDwas the greatest $\mathrm{TL}$, including acceleration and metabolic variables.

HR and Perceived Exertion: Total weekly TL (training and match) increased with $(p<0.05)$.

Differences in the daily TL across the training

Weekly microcycle (1-game week): Monday, Tuesday, Wednesday, Thursday, and Saturday or Sunday (MD).
Age of players, training day and training mode/type or sub-components week were also evident in the older age group

(U18). The amount of time engaged in low $(<50 \%$

HRmax) and high $\left(>90 \% \mathrm{HR}_{\max }\right)$ intensity activity during training and match-play was significantly lower and higher respectively in the U18 compared to the U14 group $(p<0.05)$. When comparing activity, the intensity $\left(\% \mathrm{HR}_{\max }\right)$ of field training was significantly lower compared to

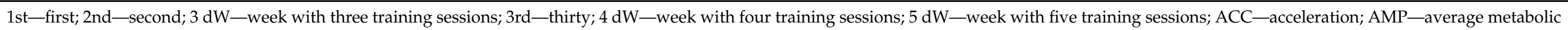

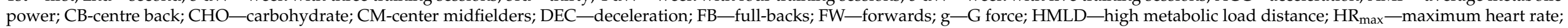

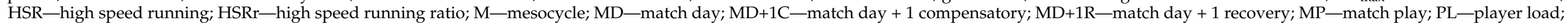

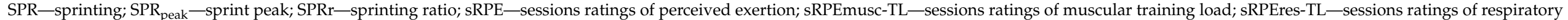
training load; TD—-total distance; TDr—total distance ratio; TL—training load; TS—training session; TSs—training sessions; U14—under-14; U18—under-18 
Table 7. Studies with predominant focus on the relationships between internal and external load.

\begin{tabular}{|c|c|c|c|c|c|}
\hline Reference (Year) & Study Purpose & Periodization Structure & Independent Variable & Main Findings & Practical Applications \\
\hline $\begin{array}{c}\text { Alexiou and Coutts } \\
\text { [52] }\end{array}$ & $\begin{array}{l}\text { Compared the sRPE method for } \\
\text { quantifying internal load with } \\
\text { various HR-based TL } \\
\text { quantification (Bannister's } \\
\text { TRIMP, LTzone TL and Edward's } \\
\text { TL) in different training modes. }\end{array}$ & $\begin{array}{l}\text { Weekly microcycle (1-game } \\
\text { week): } 3 \text { TSs technical/tactical, } \\
2 \text { TSs high-intensity resistance, } \\
1 \text { TS aerobic conditioning, } 1 \text { TS } \\
\text { core stability, 1TS pool } \\
\text { "recovery" and 1 MP. }\end{array}$ & $\begin{array}{l}\text { Training mode/type or } \\
\text { sub-components }\end{array}$ & $\begin{array}{l}\text { RPE vs. HR: Correlation for RPE and method for } \\
\text { quantifying internal load were: Bannister's } \\
\text { TRIMP }(\mathrm{r}=0.84) \text {; LT zone }(\mathrm{r}=0.83) \text {; Edwards TL } \\
(\mathrm{r}=0.85) \text {. There are differences between training } \\
\text { mode }(p<0.001) \text {; strongest correlations were } \\
\text { reported for technical }(\mathrm{r}=0.68 \text { to } 0.82) \text {, } \\
\text { conditioning }(\mathrm{r}=0.60 \text { to } 0.70) \text { and speed }(\mathrm{r}=0.61 \\
\text { to } 0.79) \text { sessions. }\end{array}$ & $\begin{array}{l}\text { sRPE method was a valuable tool to } \\
\text { internal load quantification that can } \\
\text { measure both psychological and } \\
\text { physiological factors. Therefore, sRPE } \\
\text { seems to be a more global indication of } \\
\text { the internal stress. }\end{array}$ \\
\hline $\begin{array}{l}\text { Campos-Vazquez } \\
\text { et al. [50] }\end{array}$ & $\begin{array}{c}\text { Described internal load } \\
\text { performed during a typical week } \\
\text { and determined the relationship } \\
\text { between different internal load } \\
\text { measures. }\end{array}$ & ND & $\begin{array}{l}\text { Training mode/type or } \\
\text { sub-components }\end{array}$ & $\begin{array}{l}\text { RPE vs. HR: Higher internal load during skills } \\
\text { drills/circuit training and small sided games than } \\
\text { in tactical training and pre-match activation. } \\
\text { Large relationships were found between } \\
\mathrm{HR}_{\max }>80 \% \mathrm{HR}_{\max } \text { and } \mathrm{R}>90 \% \mathrm{HR}_{\max } \text { vs. } \\
\text { sRPE }(\mathrm{r}=0.61 \text { to } 0.68) \text {. Very large relationships } \\
\text { were found between Edwards TL and sRPE and } \\
\text { between TRIMP } \\
\text { MOD and sRPE }(\mathrm{r}=0.73 \text { to } 0.87) \text {. }\end{array}$ & $\begin{array}{l}\text { Internal load variables relationships differ } \\
\text { according training mode/type. For this } \\
\text { reason, caution should be applied when } \\
\text { using RPE- or HR-derived measures to } \\
\text { quantify training or exercise intensity. }\end{array}$ \\
\hline $\begin{array}{c}\text { Casamichana et al. } \\
\text { [115] }\end{array}$ & $\begin{array}{l}\text { Examined the relationship } \\
\text { between internal and external } \\
\text { load indicators used to } \\
\text { quantify TL. }\end{array}$ & ND & $\begin{array}{l}\text { TL indicators (external } \\
\text { and internal load) }\end{array}$ & $\begin{array}{l}\text { PL vs. HR and RPE: Very-large association for PL } \\
\text { with Edward's TL }(\mathrm{r}=0.72, p<0.01) \text { and sRPE } \\
(\mathrm{r}=0.76, p<0.01) . \\
\text { TD vs. PL and RPE: Large to very-large } \\
\text { association between TD and PL }(\mathrm{r}=0.70, p<0.01) \text {, } \\
\text { sRPE }(\mathrm{r}=0.74, p<0.01) \text { and }(\mathrm{r}=0.72, p<0.01)\end{array}$ & $\begin{array}{l}\text { sRPE was a global indicator to measure } \\
\text { internal training response. Very large } \\
\text { association between PL and internal load } \\
\text { measures expresses the interest of } \\
\text { accelerations monitoring. TL analysts } \\
\text { should take advantage using GPS } \\
\text { technology and sRPE or Edwards } \\
\text { methods for post-hoc TL monitoring. }\end{array}$ \\
\hline Gaudino et al. [90] & $\begin{array}{l}\text { Compared measurements of } \\
\text { high-intensity activity during } \\
\text { field-based TS in different } \\
\text { playing positions. TD covered at } \\
\left.>14.4 \mathrm{~km} \times \mathrm{h}^{-1}\right) \text { and TP } \\
\qquad\left(>20 \mathrm{~W} \times \mathrm{kg}^{-1}\right)\end{array}$ & ND & Playing position & $\begin{array}{l}\text { TD vs. TP: Difference within TD covered at } \\
>14.4 \mathrm{~km} \times \mathrm{h}^{-1} \text { and TP was greater for central } \\
\text { defenders }(\sim 85 \%) \text { than WD and attackers }(\sim 60 \% \text {, } \\
p<0.05) \text {. Differential between methods also } \\
\text { decreased as the proportion of high-intensity } \\
\text { distance within a training session increased } \\
\left(\mathrm{R}^{2}=0.43, p<0.001\right) \text {. }\end{array}$ & $\begin{array}{l}\text { Metabolic power may provide better } \\
\text { examination for high-intensity } \\
\text { component of training which typically } \\
\text { represents the most physically } \\
\text { demanding elements. Including } \\
\text { metabolic power analysis can minimize } \\
\text { underestimation on external load } \\
\text { quantification using traditional } \\
\text { monitoring approach. }\end{array}$ \\
\hline Gaudino et al. [118] & $\begin{array}{l}\text { Identified the external load } \\
\text { measures that are most } \\
\text { influential on perceptual } \\
\text { response during } \\
\text { training sessions. }\end{array}$ & ND & TL indicators (RPE) & $\begin{array}{l}\text { RPE vs. HSR: Perceptual responses provided } \\
\text { within-individual correlations with HSR } \\
(p<0.001) \text {. } \\
\text { RPE vs. body impacts: RPE within correlated } \\
\text { with the number of impacts }(p<0.001) \text {. } \\
\text { RPE vs. ACC: Within-individual correlations } \\
\text { with ACC }(p<0.001) \text {. }\end{array}$ & $\begin{array}{l}\text { HSR, the number of impacts and } \\
\text { accelerations are the best external load } \\
\text { measures to predict perceptual response } \\
\text { during training process. Understanding } \\
\text { the influence of characteristics affecting } \\
\text { RPE may help in enhance training design } \\
\text { and athlete monitoring. }\end{array}$ \\
\hline
\end{tabular}


Table 7. Cont.

\begin{tabular}{|c|c|c|c|c|c|}
\hline Reference (Year) & Study Purpose & Periodization Structure & Independent Variable & Main Findings & Practical Applications \\
\hline $\begin{array}{c}\text { Impellizzeri et al. } \\
{[54]}\end{array}$ & $\begin{array}{l}\text { Quantified internal load using } \\
\text { sRPE and assessed correlations } \\
\text { within HR-based methods } \\
\text { (Edwards, Banister, and } \\
\text { Lucia TL). }\end{array}$ & $\begin{array}{l}\text { Weekly microcycle (1-game } \\
\text { week): Monday, Tuesday, } \\
\text { Wednesday, Thursday, and } \\
\text { Saturday (MP). Sunday and } \\
\text { Friday are days ff. } \\
\text { Typical training program was: } \\
\text { heaviest aerobic training } \\
\text { (Monday), speed developing } \\
\text { through sprint and plyometric } \\
\text { (Tuesday), running interval } \\
\text { training (2 times week), and } \\
\text { MP (Saturday). }\end{array}$ & Training day & $\begin{array}{l}\text { RPE vs. HR: Mean sRPE reported to field-based } \\
\text { training was: Monday }(32 \%) \text {, Tuesday }(27.8 \%) \text {, } \\
\text { Wednesday }(22.8 \%) \text {, and Thursday }(17.3 \%) \text {. Match } \\
\text { load corresponded to } 24 \% \text { of the total weekly TL. } \\
\text { Peak internal load was reached the first day of the } \\
\text { training week (after a day of total recovery). } \\
\text { Individual TS showed some variability on peak } \\
\text { internal TL sessions within the week. All } \\
\text { individual correlations between various HR-based } \\
\text { TL and sRPE were statistically significant ( } \mathrm{r}=0.50 \\
\text { to } 0.85, p<0.01) \text {. }\end{array}$ & $\begin{array}{l}\text { SRPE can be considered a good indicator } \\
\text { to global internal load and has potential } \\
\text { to TL quantification. The moderate } \\
\text { correlation cannot support this method as } \\
\text { a HR-based methods substitute, as only } \\
\text { about } 50 \% \text { of variance in HR was } \\
\text { explained by sRPE. }\end{array}$ \\
\hline Kelly et al. [65] & $\begin{array}{l}\text { Quantified the within-participant } \\
\text { correlations between variability } \\
\text { in sRPE and } \\
\text { HR-derived measures. }\end{array}$ & ND & Playing positions & $\begin{array}{l}\text { RPE vs. HR: The correlation between changes in } \\
\text { sRPE and Edwards TL }(\mathrm{r}=0.75) \text {. These } \\
\text { correlations across playing position: WD }(\mathrm{r}=0.81) \text {; } \\
\mathrm{CD}(\mathrm{r}=0.74) ; \mathrm{WD}(\mathrm{r}=0.70) ; \mathrm{CM}(\mathrm{r}=0.70) \text {; } \mathrm{ST} \\
(\mathrm{r}=0.84)(p<0.001) \text {. }\end{array}$ & $\begin{array}{l}\text { sRPE was a simple and practical global } \\
\text { indicator of individual TL in elite-level } \\
\text { soccer player regardless the } \\
\text { playing position. }\end{array}$ \\
\hline $\begin{array}{c}\text { Marynowicz et al. } \\
{[98]}\end{array}$ & $\begin{array}{l}\text { Examined the relationship } \\
\text { between the external TL markers } \\
\text { and the RPE and session-RPE } \\
\text { (sRPE), thereby identifying those } \\
\text { that are most influential. }\end{array}$ & ND & $\begin{array}{l}\text { TL indicators (external } \\
\text { and internal load) }\end{array}$ & $\begin{array}{l}\text { RPE vs. sRPE: Large, positive within-individual } \\
\text { correlations }(\mathrm{r}=0.62, p<0.001) \text {. } \\
\text { RPE vs. TD: Large, positive within-individual } \\
\text { correlations }(\mathrm{r}=0.70, p<0.001) \text {. } \\
\text { RPE vs. HSR: Moderate within-individual } \\
\text { correlation }(\mathrm{r}=0.39, p<0.001) \text {. } \\
\text { RPE vs. ACC: Large, positive within-individual } \\
\text { correlations }(\mathrm{r}=0.64, p<0.001) \text {. } \\
\text { RPE vs. PL: Large, positive within-individual } \\
\text { correlations ( } \mathrm{r}=0.70, p<0.001) \text {. }\end{array}$ & $\begin{array}{l}\text { The findings demonstrate that RPE does } \\
\text { not reflect the intensity of a training } \\
\text { session and that sRPE can be a useful, } \\
\text { simple, and cost-effective tool for } \\
\text { monitoring TL. Determining which } \\
\text { external load markers have the most } \\
\text { influence on the perception of effort } \\
\text { enables coaches to better monitor athletes } \\
\text { and as a consequence both reduce the risk } \\
\text { of injury and improve } \\
\text { physical performance. }\end{array}$ \\
\hline
\end{tabular}


Table 7. Cont. Study Purpose

RPE vs. HSR and SPR: Moderate correlation for perceptual responses within MSR and HSR perceptual responses within MSR and HSR quantified using the arbitrary method $(\mathrm{r}=0.53$ $0.59 ; p<0.05)$. The magnitude of correlation tended to increase when the individualized
method was used $(\mathrm{r}=0.58$ to $0.67 ; p<0.05)$. Correlation to SPR was moderate only when the
Examined the within-player correlation between perceptual

Rago et al. [107] $\quad \begin{gathered}\text { responses (RPE) and external } \\ \text { load (high-speed running using }\end{gathered}$ arbitrary and individualized speed zones).
Weekly microcycle (1-game week): "match day minus" format: MD-5, MD-4, MD-3, MD-2, MD-2, MD-1, MD. Day after MP was day-off.
Training day individualized method was used $(0.55(0.05 ; 0.83)$ and $0.53(0.02 ; 0.82), p<0.05)$.

RPE vs. HSR and SPR: Perceptual responses were largely correlated to TD within all three speed running zones, independently quantification method quantification method $(r=0.58$ to $0.68 ; p<0.05$ ). No significant correlations were observed whe external load was measured with percentage $(p>0.05)$.

\section{HR and RPE vs. TD, PL and HSR/SPR: Large}

correlation for TD, LSA volume, and PL with

Compared various measures of training load derived from

Scott et al. [57] physiological and physical data during in-season field-based training. and internal load) $0.84, p<0.01$ ) correlations. Moderate to large correlation for HSR volume and very HSR with $p<0.01$.
Adjusted values of distances covered within the TSs for individual speed being more representative of perceptual responses to training, rather than percentage of TD. Instead, splitting values of distances covered can provide better responses to the training process.

TD, LSA, and PL can be useful external load indicators to field-based training.

Physical activity measures such HSR and very HSR may provide additional information not reflected in perceptual and physiological methods.
Weekly microcycle (1-game week): Monday, Tuesday, Wednesday, Thursday, and Saturday (MP). Sunday is a day off.

Analyzed the in-season variation

Vahia et al. [57]

in correlation between HR-base method and perceptual response (sRPE).

Typical training program was: 2 technical sessions (Monday) strength training (Tuesday) resistance training (Wednesday), 1 speed and technical session (Thursday) match preparation (Friday) and MP (Saturday).
RPE vs. HR: The monthly correlations ranged from $\mathrm{r}=0.60$ to $0.73(p<0.05)$ and the overall correlation was $\mathrm{r}=0.64(0.60-0.68 ; p<0.001)$. The changes in HRTL and sRPE showed large $p<0.001)$ correlations over months $(\mathrm{r}=0.64[0.60-0.68]$
sRPE was a reliable measure to measure This method presented small variations and little bias when compared to HR-derived methods. internal load during the entire season. (mesocycle)

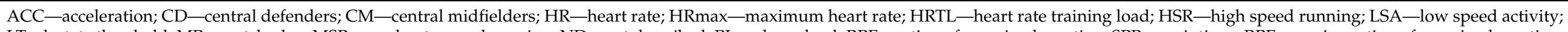

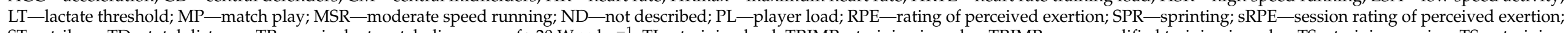

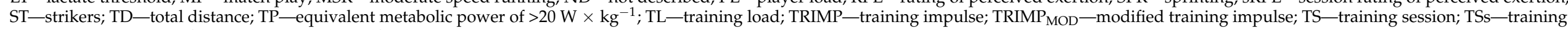
sessions; WD—wide defenders; WM—-wide midfielders. 


\section{Discussion}

The present systematic review focused on three purposes: (1) analyzing intra- and inter-individual accumulative training load distribution within week (microcycle), weeks (mesocycle) and/or season phases; (2) analyzing the intra- and inter-individual accumulative training load and match load distribution within one week (microcycle), weeks (mesocycle), and/or season phases; and (3) analyzing relationships between internal and external load measures in the accumulative training load quantification.

The findings from the reviewed studies were organized into weekly training load distribution analysis, weekly training and match load distribution analysis, and relationships between weekly internal and external distribution. Therefore, the present discussion was conducted following the independent variables of age group, match contextual factors, periodization structures (i.e., microcycles, mesocycles, and/or season phases), playing positions, training mode or sub-components, week schedule format (i.e., 1-, 2- and 3-game week), player's starting status, playing positions, and training load indicators. This systematic review ensures a general overview about monitoring daily and accumulated load. The main results demonstrated that the weekly microcycle presented a high load variation and a limited variation along season phases. Both were influenced by the type of week, player's starting status, playing positions, age group, training mode, and contextual factors.

\subsection{Weekly Training Load Distribution Analysis}

The distribution of daily and accumulated load during a weekly microcycle (1-game week) was specified by seven included studies. Of these studies, six studies employed the format «match-day (MD) minus format» (i.e., $\mathrm{MD}$ - and/or MD+) and one study subdivided the week into post-match (session after the match), pre-match (session before the match), and mid-week (remaining training sessions). The accumulated training load showed a non-perfect load pattern within weekly microcycle. On that, the literature reported the greatest intensity and volume mid-week. However, there is no consensus among reviewed studies about the training day with highest values for high-intensity movements. On the other hand, a small seasonal load variation was reported with a non-significant higher accumulated weekly physiological load during pre-season. The influence of match-related contextual variables was clearly evidenced, which requires a more individualized approach. The training mode and age-related influence should also be considered for weekly training load distribution.

Clemente et al. [88] noted an intra-week load variance. Clemente et al. [89] reported that the highest load occurred over the MD+2, MD-5, MD-4, and MD-3. The lowest load was found on the MD+1, MD-2, and MD-1. The daily and accumulated load were significantly reduced on the MD-1, with no significant differences observed in other days [56]. Oliveira et al. [72] noted conflicting findings to daily internal and external load. The external loads were similar until MD-1 while the internal load did not present the same pattern. In the same line, MD+1 provided the highest average speed and high-speed running (HSR). Contrarily, MD+1 showed the lowest session rating of perceived exertion (sRPE) score. Malone et al. [56] showed the greatest intensity and covered distances performed on the MD-5 and MD-3. Oliveira et al. [72] presented a non-perfect load pattern by decreasing values until MD-1: MD-5 > MD-4 < MD-3 > MD-2 > MD-1. It was clear that MD-5 and MD-2 provided highest high-intensity [48]. In another study, the highest values were reported on MD-3 relative to the other days (MD-4, MD-2, MD-1) [99]. As well as that, a large weekly variation was found for the same type of day. That may exceed the recommendations to progressively load increase (between 5 and 15\%) [91]. Regardless of the stage of development, Coutinho et al. [48] also observed an unloading on the MD-1. Conversely, the weekly training load distribution in the other age groups was different. The U19 showed high values of high-intensity activity in mid-week and pre-match. Moreover, U15 experienced residual weekly training load variations. The weekly external load distribution differs when comparing two teams from different countries [89]. According to Clemente et al. [89], the Portuguese team had a greater training volume on MD-2 and 
the Dutch team on MD-1. In the same study, significant differences were not found on MD-5 and MD- 4 between teams. In the same study, the number of sprints covered during training sessions were different. The Portuguese team completed more sprints on MD-5, MD-3, and MD-2, whereas the Dutch team on MD-5.

The mesocycle or week block was explained in four studies. Brito et al. [69] divided the monitoring of the seasonal training load into four different phases (preparation I, competition I, preparation II, and competition II). Loading variation was reported across the season to sRPE and weekly training load, 5-72\% and 4-48\%, respectively. The highest sRPE values were observed during match-play, especially the last phase of the season (i.e., competition II). By contrast, fatigue scores did not detect differences along the competitive season. The variation of individual fatigue scores was only reported within the weekly microcycle. As well as that, Oliveira et al. [72] showed similar outcomes with Hooper Index scores across ten mesocycles and within their respective weekly microcycles. In addition, a small seasonal load variation was reported even if there were no significant differences between mesocycles. Clemente et al. [88] reported a small increase in load through descriptive statistics. Owen et al. [99] analyzed seasonal loading using a mesocycle structure $(6 \times 1$-week blocks). No significant variations have been found along mesocycles differing from the weekly microcycle training load variation.

Two studies of this review examined the weekly training load distribution comparing pre-season versus in-season $[55,56]$. One study focused their analysis in the intra-week variations isolating pre-season and comparing two professional teams. The main findings and conclusions of these three studies were consistent with the studies that opted for the mesocycle structures [56,69,72]. Nonetheless, the study by Malone et al. [56] reported an additional in-season variation to covered distance and higher $\mathrm{HR}_{\max }$ values during the beginning of the in-season than at the midpoint and endpoint. Jeong et al. [55] noted a higher accumulated weekly physiological load during pre-season when compared to in-season.

The inter-positional variation was examined in five reviewed studies with predominantly weekly training load distribution. Akenhead et al. [53] showed that only total covered distances and ACC/DEC were able to differentiate playing positions. Conversely, HSR and sprinting showed no positional differences. The central midfielders (CM) covered more distance at low and moderate acceleration thresholds than central defenders (CD). Indeed, when expressed in relation to distance covered, the wide defenders (WD) displayed a higher ACC /DEC density than CM. In Malone et al. [56], the CM and WD presented highest and CD the lowest values to total covered distance. Oliveira et al. [72] reported no significant changes for playing positions across the mesocycles analyzed. CM covered highest total distances than other playing positions. However, the authors did not find statistical significance. Moreover, the covered distance at high-intensity threshold proved that the interposition difference only took place in the first microcycle when comparing CD versus WD and WD versus wide midfielder (WM). This suggests that WD and WM have a higher high-intensity training profile. On the other hand, Owen et al. [99] documented significant differences within playing positions, especially before the match-play. CD showed lower covered distance values in comparison to CM and WM. It should be noted that the CM presents the highest covered distance at low intensity. The WD exhibited lower velocities and perceived exertion than CM and WM. The CD covered lower total distances and sprints while the opposite was pointed to WM. The analyses set out by authors revealed a limited positional variation across weekly training load. Oliveira et al. [72] provided a limited positional variation. Indeed, differences were found within-macrocycle whereas the load remained similar at the days of weekly microcycle, with the exception of MD-1.

Two included studies analyzed the time-motion and physiological profile by young football players using training data [47,48]. Coutinho et al. [48] described the age group pattern load over a typical week. Abade et al. [47] presented the overall loading without specifying any periodization structure. There were similar findings in both studies, reporting differences in the physical and physiological demands during training sessions. 
The under-15 (U15) training sessions had the most regular activity with less physiological demands [47,48]. The under-17 (U17) displayed the highest physical and physiological stimulus and under-19 (U19) had the highest high-intensity activity [48].

The influence of match-related contextual variables was mentioned in two studies $[69,108]$. Brito et al. [69] noted that the internal load of young football players was affected by contextual factors (i.e., result of previous match, the opponent's level, and the location of the previous and following marches). According to the authors, the highest accumulated training load occurred during the training sessions after losing or drawing. By contrast, the lower loading was found before and after a match-play with a top-level opponent. After playing an away match-play, weekly training loads were higher than for a home match-play. Nonetheless, Owen et al. [108] did not report significant findings to confirm that contextual factors have an influence although the descriptive data point to a decrease in the training load after a win and away match-play. These findings are consistent with the need for a more individualized approach to initial preparation and subsequent match conditions [119,120]. Previous studies emphasize the importance of considering the independent and interactive effects of match-related contextual factor to the physical component of football performance [121,122].

The influence of the training mode, type, or sub-components were assessed in one study by weekly training load analysis. The training intensity presented associations with technical/tactical specifics and cool-down training sessions during the pre-season [55]. The contextual factors influenced the weekly training load distribution [61]. According to Rago et al. [61], the weekly TL seemed to be slightly affected by match-related contextual variables.

\subsection{Weekly Training Load and Match Load Distribution Analysis}

Two different periodization structures were explained in the studies with predominantly training and match load analysis. The weekly microcycle was reported in six studies and mesocycle (or week-blocks) were used by four authors. In this scope, the studies that included the match load also appear to show differences in the loading distribution, especially in the middle of week (i.e., MD-5, MD-4, and MD3). Limited load variation between the mesocycles were also reported. Furthermore, the type of weekly microcycle (i.e., one-, two-, and three-game week) appears to decidedly influence in the loading distribution. Additionally, the compensatory session was more intense than the recovery session. The match-related contextual factors, playing position, player's starting status, age-related influence, and training mode should also be considered for weekly training load and match load distribution analysis.

Kelly et al. [70] showed a total distance and sRPE decreased in the MD-3. The highintensity movements (HSR and sprinting) were higher in MD-3 and MD-2 than MD-1. Another study presented a progressive increase in perceived load until mid-week (i.e., MD-3) and subsequent decrease until MD-1 [68]. Martin-Garcia et al. [82] reported that the overall external load decreased progressively before match-play, especially in the MD-2 and MD-1. In agreement with the based-volume metrics, a reduced load raised in the MD-1 and MD-2 compared to MD-5, MD-3, and MD-4. Owen et al. [99] reported a significantly higher percentage for ACC/DEC values during MD-4, MD-2, and MD-1. Using a multi-modal approach, this study suggests that these metrics may provide higher levels ( $21 \%$ to $48 \%$ ) when compared to explosive movements ( $2 \%$ to $11 \%)$. The compensatory session was more intense than the recovery session [79]. Similarly, Owen et al. [99] demonstrated that the MD-4 and MD-3 were the highest intensity and volume within the weekly microcycle, revealing a weekly highest load closer to MD. Anderson et al. [87] and Sanchez-Sanchez et al. [91] verified the greatest load in MD-3 and MD-2, respectively. The MD-1 was the lowest load in various studies whereas Owen et al. [99] presented a higher ACC/DEC in MD-1 than MD-2. At amateur level, the MD+1 and MD-1 were less loading [91]. The observed main findings seemed to have been converging in a strategy tapering based on a gradual reduction until the last day before MD. 
During the competition period, the studies with training and match data seem to indicate a limited load variation between the mesocycles; similar with within playing positions. In contrast, the weekly microcycle presented the reported fluctuations in the external and internal load, which was further influenced by the playing position. Kelly et al. [70] described a slight increase in the beginning of the in-season and a small decrease along season. The total distance and SRPE was greater at the beginning of the in-season. The weekly accumulated load varied during MD-3 and MD-4 (40\%), depending on the selected training load measure and playing positions. HSR and sprinting were the metrics that presented the greatest variability within the weekly microcycle $(0.80 \%)$ [82]. The mid-season also showed a reduction in training volume [70]. On the other hand, the training time and typical weekly training load did not differ within microcycles in amateur football [91]. Wrigley et al. [60] also established that stage of development could influence variations within the weekly microcycle. These findings were similar to those verified in studies without match load [47,48].

The different type of weekly microcycle was analyzed in two reviewed studies [87,95]. Anderson et al. [87] quantified the training and match load during a one-, two-, and threegame week schedule. Clemente et al. [95] describe weekly training load variation in week with five, four, and three training sessions. A daily and accumulated load differed with the type of week schedule [87]. Clemente et al. [95] verified that the typical training intensity in the one- and two-game week schedules were compatible. However, the same did not occur in the three-game week. Therefore, the total accumulative load was lower in the one-game week schedule in comparison with the two- and three-game week schedules. Clemente et al. [95] verified that the accumulated total distances and number of ACC/DEC were three to four times higher than average match demands. The HSR and sprinting were one to two times greater than match demands. This kind of relationship between training and match load (scores/ratios) were studied in two studies included in this systematic review [95,108]. The training/match ratios varied $\sim 2$ to 4 arbitrary units (AU) considering external load. These proportions were dependent on the numbers of training sessions per week and that may infer an independence between weekly training load and match demands. The specific multi-modal approach suggested a significant variation in the volume and intensity scores across microcycles [99]. The variability match-to-match was $\sim 16-31 \%$ (i.e., HSR and sprinting). Subsequently, it is possible to ensure that these external metrics revealed a greater sensitivity regarding contextual factors and type of week. The microcycle format may improve insights on how to appropriately implement periodization during fixture congestion $[18,123]$. Indeed, the studies with training and match data also demonstrated a limitation of the accumulated load between playing positions during the season $[70,71]$. Assessing the load patterns during the weekly microcycle may provide a most accurate positional load comparison [70].

The effect of player's starting status was explored in five studies of this review. Anderson et al. [106] verified a significant effect of player's starting playing in the total distance and high-intensity activity. Generally, starters covered more running, HSR, and sprinting distances than fringes and non-starters. Similarly, large to very large differences occurred in the perceived exertion within starters and non-starters [71,97]. The competition time was the main source to these variances $[71,106]$. The pre-season and winter-break seemed to have the highest variability across playing position [71,105]. Given the consistent these findings, it is reasonable to argue that the starting status could affect physical and physiological profiles [106]. The implementation of complementary training can be a strategy to reduce variance on the non-starting status. Stevens et al. [83] described that training to non-starters was generally higher than regular training sessions. Martin-Garcia et al. [82] verified that the compensatory session may produce the greatest ACC/DEC value within the weekly microcycle. Another interest finding in this study was the marked difference in training load at $\mathrm{MD}+1$ between players completing the majority of the matchload ( $>60 \mathrm{~min}$ ) versus players with partial or no playing time $(<60 \mathrm{~min})$. 
One study aimed to quantify typical weekly training load and their content match load by under-14 (U14), under-16 (16), and under-18 (U18) football players [60]. The results proved that the training intensity and volume increased with age. Additionally, there were significant differences in the weekly loading periodization according the development stage. The weekly field-based load was higher in the U18 than U14 and U16. Moreover, the perceived exertion did not differ within age group. The U14 and U16 training process prioritized technical and physical development, while U18 focused on competition. These conclusions were similar with two other reviewed studies that included only the training data [47,48]. Importantly, the oldest age group in Wrigley et al. [60] adopted an exponential decrease (tapering). Nonetheless, Coutinho et al. [48] visualized this trend only in U17 age group knowing that this study only applies training data. According to these conclusions, it is possible that different stages of development required different load patterns.

The training mode or sub-components were analyzed for only one study, predominantly in training and match load distribution. Their findings showed that weekly field load was higher than total gym-based load [60]. These data may provide valuable information to the strength and conditioning coach about the high intensity active profiles that could be used to develop soccer-specific training drills [40].

\subsection{Relationships between Weekly Internal and External Load}

In this systematic review, the relationships between internal and external load were explained in ten studies. Of these studies, five studies reported the relationship within internal load methods, four studies analyzed internal and external load relationships, and one study compared only external load metrics. The literature evidenced positive within and betweenindividual correlations for perceived exertion, heart rate-derived measures, and external load indicators for elite female [52], semi-professional [115], elite/professional [50,65], and young amateurs $[53,98]$. The magnitude of correlations tended to increase when it was considered a within-individual correlation. The sRPE was a consistent method to quantify internal load along an entire season. The internal training load may be useful to assess accumulated training load and the relations with external training load by playing position, training mode, and/or age-groups. The reviewed studies showed a relationship between external and internal training load indicators. However, analyzing high intensity demands must take into account some considerations about speed thresholds, metabolic power output, accelerations, and accelerometers measures.

Alexiou and Coutts [52] reported positive correlation for sRPE with Banister's TRIMP, LTzone, and Edwards's TRIMP ( $\mathrm{r}=0.84, \mathrm{r}=0.83$, and $\mathrm{r}=0.85$, all $p<0.01$, respectively). Campos-Vazquez et al. [50] also reported correlations between sRPE with TRIMP MOD $_{\text {TO }}$ and Edwards TRIMP ( $\mathrm{r}=0.92$ to 0.98 ). Casamichana et al. [115] reported associations for Edwards TRIMP with sRPE ( $\mathrm{r}=0.57 p<0.01$, respectively). The correlations presented by Impellizzeri et al. [54] were statistically significant for sRPE and Edwards, Banister, and Lucia TRIMP's (from $r=0.50$ to $0.85, p<0.01$ ). Kelly et al. [65] indicated correlation between changes in SRPE and Edwards TRIMP ( $\mathrm{r}=0.75, p<0.05)$. Particularly, these main findings prove that there were correlation changes between perceived exertion and HR measures for elite female [52], semi-professional [115], elite/professional [50,65], and young amateurs $[53,98]$.

Casamichana et al. [115] reported associations for PL with Edwards TRIMP and sRPE methods $(r=0.70$ and $r=0.74$, all $p<0.05$, respectively). Total distance covered associated with PL, sRPE, and Edwards TRIMP methods ( $\mathrm{r}=0.70$ and $\mathrm{r}=0.74$, all $p<0.05$, respectively). Gaudino et al. [108] reported for RPE with HSR, impacts and accelerations $(r=0.14, r=0.09$ and $\mathrm{r}=0.25$, all $p<0.001$, respectively). Additionally, the adjusted correlation for RPE were $r=0.11, r=0.45$, and $r=0.37$, respectively. In the study by Rago et al. [107], RPE was moderately correlated to MSR and HSR using the arbitrary method $(p<0.05 ; \mathrm{r}=0.53$ to 0.59). However, the magnitude of correlations tended to increase for the individualized method ( $p<0.05 ; \mathrm{r}=0.58$ to 0.67 ). When the external load was expressed as percentage of total distance covered, no significant correlations were observed $(p>0.05)$. Scott et al. [57] 
reported significant correlations for total distance, low-speed running, and PL with the HRbased methods and sRPE $(\mathrm{r}=0.71$ to $0.84 ; p<0.01)$. The internal measures had correlation with volume of HSR and sprinting $(r=0.40$ to $0.67 ; p<0.01)$. Marynowicz et al. [98] reported a large and positive within-individual correlations for total distance, $\mathrm{PL}$, number of ACC, and $\operatorname{sRPE}(\mathrm{r}=0.70,0.64$, and 0.62 , respectively, $p<0.001)$. Small to moderate within-individual correlations were noted between RPE and measures of intensity $(r=0.16$ to 0.39). A moderate within-individual correlation was observed between HSR per minute and RPE $(\mathrm{r}=0.39, p<0.001)$.

Gaudino et al. [90] compared high-intensity activity using total distance covered at speeds $>14.4 \mathrm{~km} \times \mathrm{h}^{-1}$ and the equivalent metabolic power threshold $\left(>20 \mathrm{~W} \times \mathrm{kg}^{-1}\right)$. Measuring high-intensity movements with speed categories may underestimate the energy cost by training sessions and playing positions. Moreover, the difference between methods also decreased as the proportion of high intensity distance within a training session increased $\left(R^{2}=0.43 ; p<0.001\right)$. Therefore, metabolic power estimations may have higher precision to evaluate physical demands during training sessions.

Vahia et al. [58] was the only study that reported monthly correlations $(\mathrm{r}=0.60$ to 0.73 , $(p<0.05)$ and overall correlation $(\mathrm{r}=0.64, p<0.001)$. The correlations between SRPE and HR-load were found for all months, consequently sRPE is a consistent method to quantify internal load along an entire season.

Alexiou and Coutts [52] described correlations for sRPR and three HR-based methods by training mode (all $p<0.05)$ : technical ( $\mathrm{r}=0.68$ to 0.82$)$, conditioning $(\mathrm{r}=0.60$ to 0.79 ), and speed sessions $(r=0.61$ to 0.79). Campos-Vazquez et al. [50] also found a large and very large relations between internal methods: $\mathrm{HR}>80 \% \mathrm{HR}_{\max }$ and $\mathrm{HR}>90 \%$ $\mathrm{HR}_{\max }-\mathrm{sRPE}$ during ball-possession games, technical and tactical training $(\mathrm{r}=0.61$ to 0.68$)$; Edward's TRIMP-sRPE and between TRIMP $\mathrm{MOD}^{-s R P E}$ in sessions with ball-possession games, technical and tactical training $(r=0.73$ to 0.87$)$. The reported correlations between the different HR-based methods were always documented ( $r=0.92$ to 0.98$)$. These results provide clear evidence about the applicability of HR-based methods and SRPE to measure internal load during various training modes. However, the interchangeable application of these methods to measure load and intensity should consider the low validity to quantify neuromuscular load. Kelly et al. [70] verified correlations within playing positions (WD, $\mathrm{r}=0.81 ; \mathrm{CD}, \mathrm{r}=0.74 ; \mathrm{WM}, \mathrm{r}=0.70 ; \mathrm{CM}, \mathrm{r}=0.70$; attacker, $\mathrm{r}=0.84 ; p<0.001)$. The high magnitude of the correlations (large and large to very large) may reflect the lack of specific training for the playing position.

\subsection{Study Limitations and Future Directions}

There are some limitations that should be addressed in the practical application of this review: (i) the different methodological approaches in the reviewed studies; (ii) the related training load measures, metrics, and thresholds that have varied according to the authors; and (iii) methodological constraints about screening procedures. First, several authors point out that the most contentious limitations were the external validity of data collection. Second, future investigations should consider a meta-analytic procedure to quantify training and match load, with which data extrapolation may underestimate the daily and accumulated load. Thirty, we have considered only full-text articles available in English; this was a language limitation in the literature search strategy.

The wide range of sample sizes $(9 \leq n \leq 160)$ can influence the data comparability such as the characteristics of observations: monitoring periods (3-43 weeks), total training sessions (17-2981) and training sessions per week (3-6). Moreover, future training load analysis should be focused on different coaches, tapering strategies, and continuous seasons. This longitudinal design might include different teams and competitive levels. In the present review, only two studies compared the accumulated training load performed by two teams $[88,89]$. Most studies conducted the analysis in only one team and/or club wherefore the data may not be representative to other teams and countries. The included studies adhered to different competitive levels, geographic locations, and populations. 
More studies are needed in order to obtain greater precision in quantifying training load in different locations and competitive levels. Comparing main differences according to the competitive level can provide important information about the level and experience of the players. The studies recruited adult $(n=23)$ and young $(n=7)$ players as participants. There is a research gap on female players, given that only one study was conducted in this population [52]. Furthermore, there is no evidence-based study in the daily and accumulated load in goalkeepers, except an exploratory case study that provide a physical load report during a competitive season [123,124].

Several studies included GPS systems in their procedures with different specifications and sampling frequencies (i.e., 5, 10, and $15 \mathrm{~Hz}$ ). The validity and reliability were well documented in the literature $[25,26]$. There were some limitations when applying a sampling frequency between $1 \mathrm{~Hz}$ and $5 \mathrm{~Hz}$ during distances covered at high intensity, speedbased measures, and short linear distances with changes of direction [112]. GPS devices at $10 \mathrm{~Hz}$ seem to be the most valid and reliable systems whereas the increase in sampling frequency to $15 \mathrm{~Hz}$ does not seem to provide any additional benefit assessing team sport movements $[125,126]$. The concurrent use of a tracking system (i.e., GPS or LPM) and semiautomatic multiple-camera system (i.e., Prozone ${ }^{\circledR}$ ) to quantify training and match demand has obvious implications for the data comparability. The integration of different tracking systems is a methodological strategy applied in three reviewed articles $[70,87,106]$ but there is a moderate typical error in this kind of estimation [10].

The use of GPS technology to estimate energy expenditure during the training session may be underestimated [106]. Metabolic power variables seem to be more suitable to determine high-intensity movements than estimations based on speed [90]. The importance of including acceleration and accelerometer variables to quantify external load was well documented in the present systematic review. The accelerometer parameters including body impact, body load, player load or dynamic-stress load, and the acceleration and deceleration were supported in several reviewed studies. There was no consensus on the use of acceleration thresholds [53]. In addition, the comparison between acceleration variables measured with different tracking systems would be challenging [10]. Future research should focus on comparing demands for acceleration between training sessions and official matches-play measured with the same tracking system. Moreover, specific playing position should be taken into consideration.

The daily and accumulated load were usually lower than other team sports (e.g., Australian Football) and endurance sports [70]. The reviewed studies reported an intra-week variation and gradual reduction to MD-1 or MD-2, which means coaches' staff reduced volume and intensity during training sessions as competition approached. However, the majority of studies failed to provide any specific context associated with each training day, which may limit the application of such data. None of the reviewed studies focused on training and match load analysis in the seasonal variations during specific training interventions. Therefore, it would be interesting to discover what training modes, subcomponents, and exercise typologies contribute to increases or decreases (fluctuations) in certain load measures [127,128]. In team sports (in this case, football), there are some methodological challenges in training load quantification. However, it is not possible to argue that there was a direct causal relationship between physical performance and/or team performance. The dynamic and unpredictable nature of match-play may make it impossible to adjust training and matches [95]. This fact limited the understanding of the relationship between training periodization and individual and team performance [129].

The loading discrepancies within playing positions may significantly affect individual performance and increase injury risk [33]. Therefore, quantifying training load can adjust training periodization models and individualized training sessions. Additionally, Owen et al. [99] allowed the possibility of describing the daily and positional variations through the multi-modal mechanical approach. The content and magnitude of the complementary training sessions were not reported in the literature; wherefore, future investigations about training mode or sub-components effect are recommended. Martin-Garcia et al. [82] noted 
that future studies should implement mixed small-sided games and running exercise strategies to infer the greatest training stimulus for players with limited playing time (i.e., non-starters and/or fringe player).

The training load quantification in youth football suggested that as players grow older, the training process focus moves to competition whereas in younger players, the training goals were physical and technical development $[47,48,60]$. Therefore, the weekly microcycle should be adjusted for age. The influence of different weekly microcycle schedules has not yet been established for the competitive performance and long-term development of youth football players. The youth training responses differed markedly from adult and professional players' due to the development stage of sport specified-skills and physical attributes [130].

The relationships between internal and external load should be interpreted with regard to some limitations. According to Impellizzeri et al. [54], only 50\% of HR loading variation were supported by sRPE. However, there is a limitation inherent in the use of HR-based measures to quantify training intensity during anaerobic efforts. This fact may influence the magnitude of the correlation between perceived exertion and HR loading. The perceived exertion appears to be better linked with external load when the speed zones were individually determined than when the arbitrary speed zones [107]. Notwithstanding, there were some limitations in the achievement of real individual maximal values (e.g., maximal aerobic speed) and the speed zone transition. The speed zone transition is very important due to the significant physiological effort associated [131].

\section{Conclusions}

The present systematic review provided the first report about monitoring accumulated training and match load in football players. Current research suggests that the training and match load variation seem to be influenced by the type of weekly schedule, player's starting status, playing positions, age group, training mode, and contextual factors. Therefore, there was a related high variation in the weekly loading distribution and a limited load variation during a competitive season. Most of the evidence has implications for adult male professional football players concerning the large body of quantitative studies (QS: 0.65-0.89). In youth football, the studies appear to indicate a small fluctuation across weekly and seasonal accumulated load. However, further studies are recommended to improve knowledge on the female and youth accumulated training/match load monitoring.

Author Contributions: Conceptualization, J.E.T. and P.F.; data curation, J.E.T., J.R. and P.F.; formal analysis, P.F., M.L. and R.F.; funding acquisition, J.E.T., P.F. and A.M.M.; investigation, J.E.T.; methodology, P.F., T.M.B. and A.M.M.; resources, P.F. and A.M.M.; software, J.E.T., J.R. and M.L.; supervision, A.J.S. and A.M.M.; validation, P.F., T.M.B. and A.M.M.; writing-original draft, J.E.T.; writing-review and editing, P.F., R.F., A.J.S., T.M.B. and A.M.M. All authors have read and agreed to the published version of the manuscript.

Funding: This research was supported by the Douro Higher Institute of Educational Sciences and the Portuguese Foundation for Science and Technology, I.P. (project UIDB04045/2021).

Institutional Review Board Statement: The experimental approach was approved and followed by the local Ethical Committee from University of Trás-os-Montes e Alto Douro (Doc2-CE-UTAD-2021).

Informed Consent Statement: Informed consent was obtained from all subjects involved in the study.

Data Availability Statement: Data is available under request to the contact author.

Acknowledgments: The authors express acknowledgement of all coaches and playing staff for cooperation during all collection procedures.

Conflicts of Interest: The authors declare no potential conflict of interest. 


\section{References}

1. Bangsbo, J.; Mohr, M.; Krustrup, P. Physical and metabolic demands of training and match-play in the elite football player. J. Sports Sci. 2006, 24, 665-674. [CrossRef]

2. Stølen, T.; Chamari, K.; Castagna, C.; Wisløff, U. Physiology of soccer: An update. Sports Med. 2005, 35, 501-536. [CrossRef]

3. Coutts, A.; Kempton, T.; Crowcroft, S.; Coutts, A.J.; Crowcroft, S.; Kempton, T. Developing athlete monitoring systems: Theoretical basis and practical applications. In Sport, Recovery and Performance: Interdisciplinary Insights; Kellmann, M., Beckmann, J., Eds.; Routledge: Abingdon, UK, 2018; pp. 19-32.

4. Impellizzeri, F.M.; Rampinini, E.; Marcora, S.M. Physiological assessment of aerobic training in soccer. J. Sports Sci. 2005, 23, 583-592. [CrossRef]

5. Bourdon, P.C.; Cardinale, M.; Murray, A.; Gastin, P.; Kellmann, M.; Varley, M.C.; Gabbett, T.J.; Coutts, A.J.; Burgess, D.J.; Gregson, W.; et al. Monitoring athlete training loads: Consensus statement. Int. J. Sports Physiol. Perform. 2017, 12, S2-S161. [CrossRef]

6. Reilly, T. The Science of Training Soccer: A Scientific Approach to Developing Strength, Speed and Endurance; Routledge: London, UK, 2006.

7. Impellizzeri, F.M.; Marcora, S.M.; Coutts, A.J. Internal and external training load: 15 years on. Int. J. Sports Physiol. Perform. 2019, 14, 270-273. [CrossRef]

8. Cummins, C.; McLean, B.; Halaki, M.; Orr, R. Positional differences in external on-field load during specific drill classifications over a professional rugby league preseason. Int. J. Sports Physiol. Perform. 2017, 12, 764-776. [CrossRef]

9. Gómez-Carmona, C.D.; Pino-Ortega, J.; Sánchez-Ureña, B.; Ibáñez, S.J.; Rojas-Valverde, D. Accelerometry-based external load indicators in sport: Too many options, same practical outcome? Int. J. Environ. Res. Public Health 2019, 16, 5101. [CrossRef] [PubMed]

10. Buchheit, M.; Allen, A.; Poon, T.K.; Modonutti, M.; Gregson, W.; Di Salvo, V. Integrating different tracking systems in football: Multiple camera semi-automatic system, local position measurement and GPS technologies. J. Sports Sci. 2014, 32, 1844-1857. [CrossRef] [PubMed]

11. Djaoui, L.; Haddad, M.; Chamari, K.; Dellal, A. Monitoring training load and fatigue in soccer players with physiological markers. Physiol. Behav. 2017, 181, 86-94. [CrossRef] [PubMed]

12. Borresen, J.; Lambert, M.I. The quantification of training load, the training response and the effect on performance. Sports Med. 2009, 39, 779-795. [CrossRef] [PubMed]

13. Branquinho, L.; Ferraz, R.; Travassos, B.; Marinho, D.A.; Marques, M.C. Effects of Different Recovery Times on Internal and External Load during Small-Sided Games in Soccer. Sports Health 2021. [CrossRef]

14. Mujika, I.; Halson, S.; Burke, L.M.; Balagué, G.; Farrow, D. An integrated, multifactorial approach to periodization for optimal performance in individual and team sports. Int. J. Sports Physiol. Perform. 2018, 13, 538-561. [CrossRef] [PubMed]

15. Akubat, I.; Barrett, S.; Abt, G. Integrating the internal and external training loads in soccer. Int. J. Sports Physiol. Perform. 2014, 9 , 457-462. [CrossRef]

16. Akenhead, R.; Nassis, G.P. Training load and player monitoring in high-level football: Current practice and perceptions. Int. J. Sports Physiol. Perform. 2016, 11, 587-593. [CrossRef] [PubMed]

17. Halson, S.L. Monitoring training load to understand fatigue in athletes. Sports Med. 2014, 44, 139-147. [CrossRef] [PubMed]

18. Vanrenterghem, J.; Nedergaard, N.J.; Robinson, M.A.; Drust, B. Training load monitoring in team sports: A novel framework separating physiological and biomechanical load-adaptation pathways. Sports Med. 2017, 47, 2135-2142. [CrossRef]

19. Fox, J.L.; Stanton, R.; Sargent, C.; Wintour, S.-A.; Scanlan, A.T. The association between training load and performance in team sports: A systematic review. Sports Med. 2018, 48, 2743-2774. [CrossRef]

20. McLaren, S.J.; Macpherson, T.W.; Coutts, A.J.; Hurst, C.; Spears, I.R.; Weston, M. The relationships between internal and external measures of training load and intensity in team sports: A meta-analysis. Sports Med. 2018, 48, 641-658. [CrossRef]

21. Jaspers, A.; Brink, M.S.; Probst, S.G.M.; Frencken, W.G.P.; Helsen, W.F. Relationships between training load indicators and training outcomes in professional soccer. Sports Med. 2017, 47, 533-544. [CrossRef] [PubMed]

22. Silva, J.R.; Rumpf, M.C.; Hertzog, M.; Castagna, C.; Farooq, A.; Girard, O.; Hader, K. Acute and residual soccer match-related fatigue: A systematic review and meta-analysis. Sports Med. 2018, 48, 539-583. [CrossRef] [PubMed]

23. Hader, K.; Rumpf, M.C.; Hertzog, M.; Kilduff, L.P.; Girard, O.; Silva, J.R. Monitoring the athlete match response: Can external load variables predict post-match acute and residual fatigue in soccer? A systematic review with meta-analysis. Sports Med. 2019, 5, 48. [CrossRef]

24. Drew, M.K.; Finch, C.F. The relationship between training load and injury, illness and soreness: A systematic and literature review. Sports Med. 2016, 46, 861-883. [CrossRef] [PubMed]

25. Whitehead, S.; Till, K.; Weaving, D.; Jones, B. The use of microtechnology to quantify the peak match demands of the football codes: A systematic review. Sports Med. 2018, 48, 2549-2575. [CrossRef]

26. Dellaserra, C.L.; Gao, Y.; Ransdell, L. Use of integrated technology in team sports: A review of opportunities, challenges, and future directions for athletes. J. Strength Cond Res. 2014, 28, 556-573. [CrossRef] [PubMed]

27. Rago, V.; Brito, J.; Figueiredo, P.; Costa, J.; Barreira, D.; Krustrup, P.; Rebelo, A. Methods to collect and interpret external training load using microtechnology incorporating GPS in professional football: A systematic review. Res. Sports Med. 2020, 28, 437-458. [CrossRef] [PubMed] 
28. Palucci Vieira, L.H.; Carling, C.; Barbieri, F.A.; Aquino, R.; Santiago, P.R.P. Match running performance in young soccer players: A systematic review. Sports Med. 2019, 49, 289-318. [CrossRef] [PubMed]

29. Jones, C.M.; Griffiths, P.C.; Mellalieu, S.D. Training load and fatigue marker associations with injury and illness: A systematic review of longitudinal studies. Sports Med. 2017, 47, 943-974. [CrossRef] [PubMed]

30. Rampinini, E.; Coutts, A.J.; Castagna, C.; Sassi, R.; Impellizzeri, F.M. Variation in top level soccer match performance. Int. J. Sports Med. 2007, 28, 1018-1024. [CrossRef] [PubMed]

31. Bradley, P.S.; Carling, C.; Gomez Diaz, A.; Hood, P.; Barnes, C.; Ade, J.; Boddy, M.; Krustrup, P.; Mohri, M. Match performance and physical capacity of players in the top three competitive standards of English professional soccer. Hum. Mov. Sci. 2013, 32, 808-821. [CrossRef] [PubMed]

32. Mohr, M.; Krustrup, P.; Bangsbo, J. Match performance of high-standard soccer players with special reference to development of fatigue. J. Sports Sci. 2003, 21, 519-528. [CrossRef]

33. Di Salvo, V.; Baron, R.; Tschan, H.; Calderon Montero, F.J.; Bachl, N.; Pigozzi, F. Performance characteristics according to playing position in elite soccer. Int. J. Sports Med. 2007, 28, 222-227. [CrossRef] [PubMed]

34. Rebelo, A.; Brito, J.; Seabra, A.; Oliveira, J.; Drust, B.; Krustrup, P. A new tool to measure training load in soccer training and match play. Int. J. Sports Med. 2012, 33, 297-304. [CrossRef] [PubMed]

35. Issurin, V.B. New horizons for the methodology and physiology of training periodization. Sports Med. 2010, 40, 189-206. [CrossRef] [PubMed]

36. Kiely, J. Periodization paradigms in the 21st century: Evidence-led or tradition-driven? Int. J. Sports Physiol. Perform. 2012, 7, 242-250. [CrossRef]

37. Castagna, C.; Varley, M.; Póvoas, S.C.A.; D'Ottavio, S. Evaluation of the match external load in soccer: Methods comparison. Int. J. Sports Physiol. Perform. 2017, 12, 490-495. [CrossRef] [PubMed]

38. Hill-Haas, S.V.; Dawson, B.; Impellizzeri, F.M.; Coutts, A.J. Physiology of small-sided games training in football. Sports Med. 2011, 41, 199-220. [CrossRef] [PubMed]

39. Engel, F.A.; Ackermann, A.; Chtourou, H.; Sperlich, B. High-intensity interval training performed by young athletes: A systematic review and meta-analysis. Front Physiol. 2018, 27, 1012. [CrossRef] [PubMed]

40. Bradley, P.S.; Sheldon, W.; Wooster, B.; Olsen, P.; Boanas, P.; Krustrup, P. High-intensity running in English FA Premier League soccer matches. J. Sports Sci. 2009, 27, 159-168. [CrossRef] [PubMed]

41. Elkins, M.R.; Herbert, R.D.; Moseley, A.M.; Sherrington, C.; Maher, C. Rating the quality of trials in systematic reviews of physical therapy interventions. Cardiopulm. Phys. Ther. J. 2010, 21, 20-26. [CrossRef] [PubMed]

42. Moher, D.; Liberati, A.; Tetzlaff, J.; Altman, D.G. Preferred reporting items for systematic reviews and meta-analyses: The PRISMA statement. BMJ 2009, 339, b2535. [CrossRef]

43. Vandenbroucke, J.P.; von Elm, E.; Altman, D.G.; Gøtzsche, P.C.; Mulrow, C.D.; Pocock, S.J.; Poole, C.; Schlesselman, J.J.; Egger, M.; STROBE Initiative. Strengthening the reporting of observational studies in epidemiology (STROBE): Explanation and elaboration. Int. J. Surg. 2014, 12, 1500-1524. [CrossRef]

44. Von Elm, E.; Altman, D.G.; Egger, M.; Pocock, S.J.; Gøtzsche, P.C.; Vandenbroucke, J.P. The strengthening the reporting of observational studies in epidemiology (STROBE) statement: Guidelines for reporting observational studies. Lancet 2007, 370, 1453-1457. [CrossRef]

45. Falck, R.S.; Davis, J.C.; Liu-Ambrose, T. What is the association between sedentary behaviour and cognitive function? A systematic review. Br. J. Sports Med. 2017, 51, 800-811. [CrossRef] [PubMed]

46. Silva, A.F.; Conte, D.; Clemente, F.M. Decision-Making in Youth Team-Sports Players: A Systematic Review. Int. J. Environ. Res. Public Helath 2020, 17, 3803. [CrossRef] [PubMed]

47. Abade, E.A.; Gonçalves, B.V.; Leite, N.M.; Sampaio, J.E. Time-motion and physiological profile of football training sessions performed by under-15, under-17 and under-19 elite Portuguese players. Int. J. Sports Physiol. Perform. 2014, 9, 463-470. [CrossRef]

48. Coutinho, D.; Gonçalves, B.; Figueira, B.; Abade, E.; Marcelino, R.; Sampaio, J. Typical weekly workload of under 15, under 17, and under 19 elite Portuguese football players. J. Sports Sci. 2015, 33, 1229-1237. [CrossRef]

49. Gore, C. Physiological Tests for Elite Athletes; Human Kinetics: Champaign, IL, USA, 2000.

50. Campos-Vazquez, M.A.; Toscano-Bendala, F.J.; Mora-Ferrera, J.C.; Suarez-Arrones, L.J. Relationship between internal load indicators and changes on intermittent performance after the preseason in professional soccer players. J. Strength Cond. Res. 2017, 31, 1477-1485. [CrossRef]

51. Stagno, K.M.; Thatcher, R.; van Someren, K.A. A modified TRIMP to quantify the in-season training load of team sport players. J. Sports Sci. 2007, 25, 629-634. [CrossRef] [PubMed]

52. Alexiou, H.; Coutts, A.J. A comparison of methods used for quantifying internal training load in women soccer players. Int. J. Sports Physiol. Perform. 2008, 3, 320-330. [CrossRef]

53. Akenhead, R.; Harley, J.A.; Tweddle, S.P. Examining the external training load of an English premier league football team with special reference to acceleration. J. Strength Cond. Res. 2016, 30, 2424-2432. [CrossRef] [PubMed]

54. Impellizzeri, F.M.; Rampinini, E.; Coutts, A.J.; Sassi, A.; Marcora, S.M. Use of RPE-based training load in soccer. Med. Sci. Sports Exerc. 2004, 36, 1042-1047. [CrossRef]

55. Jeong, T.-S.; Reilly, T.; Morton, J.; Bae, S.-W.; Drust, B. Quantification of the physiological loading of one week of 'pre-season' and one week of 'in-season' training in professional soccer players. J. Sports Sci. 2011, 29, 1161-1166. [CrossRef] 
56. Malone, J.J.; Di Michele, R.; Morgans, R.; Burgess, D.; Morton, J.P.; Drust, B. Seasonal training-load quantification in elite English Premier League soccer players. Int. J. Sports Physiol. Perform. 2015, 10, 489-497. [CrossRef]

57. Scott, B.R.; Lockie, R.G.; Knight, T.J.; Clark, A.C.; Janse de Jonge, X.A. A comparison of methods to quantify the in-season training load of professional soccer players. Int. J. Sports Physiol. Perform. 2013, 8, 195-202. [CrossRef] [PubMed]

58. Vahia, D.; Kelly, A.; Knapman, H.; Williams, C.A. Variation in the correlation between heart rate and session rating of perceived exertion-based estimations of internal training load in youth soccer players. Pediatr. Exerc. Sci. 2019, 31, 91-98. [CrossRef] [PubMed]

59. Dalen, T.; Lorås, H. Monitoring Training and Match Physical Load in Junior Soccer Players: Starters versus Substitutes. Sports 2019, 7, 70. [CrossRef] [PubMed]

60. Wrigley, R.; Drust, B.; Stratton, G.; Scott, M.; Gregson, W. Quantification of the typical weekly in-season training load in elite junior soccer players. J. Sports Sci. 2012, 30, 1573-1580. [CrossRef] [PubMed]

61. Rago, V.; Rebelo, A.; Krustrup, P.; Mohr, M. Contextual Variables and Training Load Throughout a Competitive Period in a Top-Level Male Soccer Team. J. Strength Cond. Res. 2019. [CrossRef] [PubMed]

62. Bangsbo, J.; Iaia, F.M.; Krustrup, P. The Yo-Yo intermittent recovery test: A useful tool for evaluation of physical performance in intermittent sports. Sports Med. 2008, 38, 37-51. [CrossRef] [PubMed]

63. Banister, E. Modeling elite athletic performance. In Physiological Testing of Elite Athletes; Green, H.J., McDougal, J.D., Wegner, H.A., Eds.; Human Kinetics: Champaign, IL, USA, 1991; pp. 403-424.

64. Foster, C.; Florhaug, J.A.; Franklin, J.; Gottschall, L.; Hrovatin, L.A.; Parker, S.; Doleshal, P.; Dodge, C. A new approach to monitoring exercise training. J. Strength Cond. Res. 2001, 15, 109-115. [PubMed]

65. Kelly, D.M.; Strudwick, A.J.; Atkinson, G.; Drust, B.; Gregson, W. The within-participant correlation between perception of effort and heart rate-based estimations of training load in elite soccer players. J. Sports Sci. 2016, 34, 1328-1332. [CrossRef]

66. Edwards, S. High performance training and racing. In The Heart Rate Monitor Book; Edward, S., Ed.; Feet Fleet Press: Sacramento, CA, USA, 1993; pp. 113-123.

67. Lucia, A.; Hoyos, J.; Santalla, A.; Earnest, C.; Chicharro, J.L. Tour de France versus Vuelta a España: Which is harder? Med. Sci. Sports Exerc. 2003, 35, 872-878.

68. Hoff, J.; Wisløff, U.; Engen, L.C.; Kemi, O.J.; Helgerud, J. Soccer specific aerobic endurance training. Br. J. Sports Med. 2002, 36, 218-221. [CrossRef]

69. Brito, J.; Hertzog, M.; Nassis, G.P. Do match-related contextual variables influence training load in highly trained soccer players? J. Strength Cond. Res. 2016, 30, 393-399. [CrossRef] [PubMed]

70. Kelly, D.M.; Strudwick, A.J.; Atkinson, G.; Drust, B.; Gregson, W. Quantification of training and match-load distribution across a season in elite English Premier League soccer players. Sci. Med. Footb. 2019, 4, 59-67. [CrossRef]

71. Los Arcos, A.; Mendez-Villanueva, A.; Martínez-Santos, R. In-season training periodization of professional soccer players. Biol. Sport. 2017, 34, 149-155. [CrossRef] [PubMed]

72. Oliveira, R.; Brito, J.P.; Martins, A.; Mendes, B.; Marinho, D.A.; Ferraz, R.; Marques, M.C. In-season internal and external training load quantification of an elite European soccer team. PLoS ONE 2019, 14, e0209393. [CrossRef] [PubMed]

73. Borg, G.; Hassmén, P.; Lagerström, M. Perceived exertion related to heart rate and blood lactate during arm and leg exercise. Eur. J. Appl. Physiol. Occup. Physiol. 1987, 56, 679-685. [CrossRef]

74. Foster, C. Monitoring training in athletes with reference to overtraining syndrome. Med. Sci. Sports Exerc. 1998, 30, 1164-1168. [CrossRef] [PubMed]

75. Haddad, M.; Stylianides, G.; Djaoui, L.; Dellal, A.; Chamari, K. Session-RPE method for training load monitoring: Validity, ecological usefulness, and influencing factors. Front. Neurosci. 2017, 11, 612. [CrossRef]

76. Los Arcos, A.; Méndez-Villanueva, A.; Yanci, J.; Martínez-Santos, R. Respiratory and muscular perceived exertion during official games in professional soccer players. Int. J. Sports Physiol. Perform. 2016, 11, 301-304. [CrossRef]

77. Los Arcos, A.; Yanci, J.; Mendiguchia, J.; Gorostiaga, E.M. Rating of muscular and respiratory perceived exertion in professional soccer players. J. Strength Cond. Res. 2014, 28, 3280-3288. [CrossRef]

78. Chatard, J.-C.; Atlaoui, D.; Pichot, V.; Gourné, C.; Duclos, M.; Guezennec, Y.-C. Training follow up by questionnaire fatigue, hormones and heart rate variability measurements. Sci. Sports 2003, 18, 302-304. [CrossRef]

79. Hooper, S.L.; Mackinnon, L.T. Monitoring overtraining in athletes. Sports Med. 1995, 20, 321-327. [CrossRef] [PubMed]

80. Aguiar, M.V.; Botelho, G.M.; Gonçalves, B.S.; Sampaio, J.E. Physiological responses and activity profiles of football small-sided games. J. Strength Cond. Res. 2013, 27, 1287-1294. [CrossRef]

81. Hill-Haas, S.; Rowsell, G.; Coutts, A.; Dawson, B. The reproducibility of physiological responses and performance profiles of youth soccer players in small-sided games. Int. J. Sports Physiol. Perform. 2008, 3, 393-396. [CrossRef] [PubMed]

82. Martin-Garcia, A.S.; Diaz, A.G.; Bradley, P.S.; Morera, F.; Casamichana, D. Quantification of a professional football team's external load using a microcycle structure. J. Strength Cond. Res. 2018, 32, 3511-3518. [CrossRef] [PubMed]

83. Stevens, T.G.; de Ruiter, C.J.; Twisk, J.W.R.; Savelsbergh, G.J.P.; Beek, P.J. Quantification of in-season training load relative to match load in professional Dutch Eredivisie football players. Sci. Med. Football. 2017, 1, 117-125. [CrossRef]

84. Minetti, A.E.; Gaudino, P.; Seminati, E.; Cazzola, D. The cost of transport of human running is not affected, as in walking, by wide acceleration/deceleration cycles. J. Appl. Physiol. 2013, 114, 498-503. [CrossRef] [PubMed] 
85. Osgnach, C.; Poser, S.; Bernardini, R.; Rinaldo, R.; Di Prampero, P.E. Energy cost and metabolic power in elite soccer: A new match analysis approach. Med. Sci. Sports Exerc. 2010, 42, 170-178. [CrossRef]

86. Rampinini, E.; Alberti, G.; Fiorenza, M.; Riggio, M.; Sassi, R.; Borges, T.O.; Coutts, A.J. Accuracy of GPS devices for measuring high-intensity running in field-based team sports. Int. J. Sports Med. 2015, 36, 49-53. [CrossRef] [PubMed]

87. Anderson, L.; Orme, P.; Di Michele, R.; Close, G.L.; Morgans, R.; Drust, B.; Morton, J.P. Quantification of training load during one-, two- and three-game week schedules in professional soccer players from the English Premier League: Implications for carbohydrate periodisation. J. Sports Sci. 2016, 34, 1250-1259. [CrossRef] [PubMed]

88. Clemente, F.M.; Seerden, G.; van der Linden, C.M. Quantifying the physical loading of five weeks of pre-season training in professional soccer teams from Dutch and Portuguese leagues. Physiol. Behav. 2019, 209, 112588. [CrossRef]

89. Clemente, F.M.; Owen, A.; Serra-Olivares, J.; Nikolaidis, P.T.; van der Linden, C.M.I.; Mendes, B. Characterization of the weekly external load profile of professional soccer teams from Portugal and The Netherlands. J. Hum. Kinet. 2019, 66, 155-164. [CrossRef]

90. Gaudino, P.; Iaia, F.M.; Alberti, G.; Strudwick, A.J.; Atkinson, G.; Gregson, W. Monitoring training in elite soccer players: Systematic bias between running speed and metabolic power data. Int. J. Sports Med. 2013, 34, 963-968. [CrossRef]

91. Sanchez-Sanchez, J.; Hernández, D.; Martin, V.; Sanchez, M.; Casamichana, D.; Rodriguez-Fernandez, A.; Ramirez-Campillo, R.; Nakamura, F.Y. Assessment of the external load of amateur soccer players during four consecutive training microcycles in relation to the external load during the official match. Mot. Rev. Educ. Física 2019, 25, e101938. [CrossRef]

92. Di Salvo, V.; Gregson, W.; Atkinson, G.; Tordoff, P.; Drust, B. Analysis of high intensity activity in Premier League soccer. Int. J. Sports Med. 2009, 30, 205-212. [CrossRef] [PubMed]

93. Bradley, P.S.; Di Mascio, M.; Peart, D.; Olsen, P.; Sheldon, B. High-intensity activity profiles of elite soccer players at different performance levels. J. Strength Cond. Res. 2010, 24, 2343-2351. [CrossRef]

94. Gregson, W.; Drust, B.; Atkinson, G.; Salvo, V. Match-to-match variability of high-speed activities in Premier League soccer. Int. J. Sports Med. 2010, 31, 237-242. [CrossRef]

95. Clemente, F.M.; Rabbani, A.; Conte, D.; Castillo, D.; Afonso, J.; Truman Clark, C.C.; Nikolaidis, P.T.; Rosemann, T.; Knechtle, B. Training/match external load ratios in professional soccer players: A full-season study. Int. J. Environ. Res. Public Health 2019, 16, 3057. [CrossRef] [PubMed]

96. Dwyer, D.B.; Gabbett, T.J. Global positioning system data analysis: Velocity ranges and a new definition of sprinting for field sport athletes. J. Strength Cond. Res. 2012, 26, 818-824. [CrossRef] [PubMed]

97. Baptista, I.; Johansen, D.; Figueiredo, P.; Rebelo, A.; Pettersen, S.A. Positional Differences in Peak- and Accumulated- Training Load Relative to Match Load in Elite Football. Sports 2020, 8, 1. [CrossRef]

98. Marynowicz, J.; Kikut, K.; Lango, M.; Horna, D.; Andrzejewski, M. Relationship between the Session-RPE and External Measures of Training Load in Youth Soccer Training. J. Strength Cond. Res. 2020, 34, 2800-2804. [CrossRef] [PubMed]

99. Owen, A.L.; Djaoui, L.; Newton, M.; Malone, S.; Mendes, B. A contemporary multi-modal mechanical approach to training monitoring in elite professional soccer. Sci. Med. Footb. 2017, 1, 216-221. [CrossRef]

100. Dellal, A.; Chamari, K.; Wong, D.P.; Ahmaidi, S.; Keller, D.; Barros, R.; Bisciotti, G.N.; Carling, C. Comparison of physical and technical performance in European soccer match-play: FA Premier League and La Liga. Eur. J. Sport Sci. 2011, 11, 51-59. [CrossRef]

101. Rampinini, E.; Bosio, A.; Ferraresi, I.; Petruolo, A.; Morelli, A.; Sassi, A. Match-related fatigue in soccer players. Med. Sci. Sports Exerc. 2011, 43, 2161-2170. [CrossRef] [PubMed]

102. Dalen, T.; Ingebrigtsen, J.; Ettema, G.; Hjelde, G.H.; Wisløff, U. Player Load, Acceleration, and Deceleration During Forty-Five Competitive Matches of Elite Soccer. J. Strength Cond. Res. 2016, 30, 351-359. [CrossRef]

103. Ingebrigtsen, J.; Dalen, T.; Hjelde, G.H.; Drust, B.; Wisløff, U. Acceleration and sprint profiles of a professional elite football team in match play. Eur. J. Sport Sci. 2015, 15, 101-110. [CrossRef] [PubMed]

104. Beato, M.; Devereux, G.; Stiff, A. Validity and reliability of global positioning system units (STATSports Viper) for measuring distance and peak speed in sports. J. Strength Cond. Res. 2018, 32, 2831-2837. [CrossRef]

105. Clemente, F.M.; Silva, R.; Castillo, D.; Los Arcos, A.; Mendes, B.; Afonso, J. Weekly Load Variations of Distance-Based Variables in Professional Soccer Players: A Full-Season Study. Int. J. Environ. Res. Public Health 2020, 17, 3300. [CrossRef] [PubMed]

106. Anderson, L.; Orme, P.; Di Michele, R.; Close, G.L.; Milsom, J.; Morgans, R.; Drust, B.; Morton, J.P. Quantification of seasonal-long physical load in soccer players with different starting status from the English Premier League: Implications for maintaining squad physical fitness. Int. J. Sports Physiol. Perform. 2016, 11, 1038-1046. [CrossRef]

107. Rago, V.; Brito, J.; Figueiredo, P.; Krustrup, P.; Rebelo, A. Relationship between external load and perceptual responses to training in professional football: Effects of quantification method. Sports 2019, 7, 68. [CrossRef] [PubMed]

108. Owen, A.L.; Lago-Peñas, C.; Gómez, M.-Á.; Mendes, B.; Dellal, A. Analysis of a training mesocycle and positional quantification in elite European soccer players. Int. J. Sports Sci. Coach. 2017, 12, 665-676. [CrossRef]

109. Di Salvo, V.; Adam, C.; Barry, M.; Marco, C. Validation of Prozone ${ }^{\circledR}$ : A new video-based performance analysis system. Int. J. Perform. Anal. Sport 2006, 6, 108-119.

110. Swallow, W.E.; Skidmore, N.; Page, R.M.; Malone, J.J. An examination of in-season external training load in semi-professional soccer players: Considerations of one and two match weekly microcycles. Int. J. Sports Sci. Coach. 2020. [CrossRef]

111. Johnston, R.J.; Watsford, M.L.; Pine, M.J.; Spurrs, R.W. Standardisation of acceleration zones in professional field sport athletes. Int. J. Sports Sci. Coach. 2014, 9, 1161-1168. [CrossRef] 
112. Malone, J.J.; Lovell, R.; Varley, M.C.; Coutts, A.J. Unpacking the Black Box: Applications and Considerations for Using GPS Devices in Sport. Int. J. Sports Physiol. Perform. 2017, 12 (Suppl. 2), S218-S226. [CrossRef] [PubMed]

113. McLellan, C.P.; Lovell, D.I.; Gass, G.C. biochemical and endocrine responses to impact and collision during elite rugby league match play. J. Strength Cond. Res. 2011, 25, 1553-1562. [CrossRef]

114. Lovell, T.W.J.; Sirotic, A.C.; Impellizzeri, F.M.; Coutts, A.J. Factors affecting perception of effort (session rating of perceived exertion) during rugby league training. Int. J. Sports Physiol. Perform. 2013, 8, 62-69. [CrossRef] [PubMed]

115. Casamichana, D.; Castellano, J.; Calleja-Gonzalez, J.; San Román, J.; Castagna, C. Relationship between indicators of training load in soccer players. J. Strength Cond. Res. 2013, 27, 369-374. [CrossRef] [PubMed]

116. Nikolaidis, P.T.; Clemente, F.M.; van der Linden, C.M.I.; Rosemann, T.; Knechtle, B. Validity and reliability of $10-\mathrm{Hz}$ global positioning system to assess in-line movement and change of direction. Front. Physiol. 2018, 9, 228. [CrossRef] [PubMed]

117. Boyd, L.J.; Ball, K.; Aughey, R.J. The reliability of MinimaxX accelerometers for measuring physical activity in Australian football. Int. J. Sports Physiol. Perform. 2011, 6, 311-321. [CrossRef] [PubMed]

118. Gaudino, P.; Iaia, F.M.; Strudwick, A.J.; Hawkins, R.D.; Alberti, G.; Atkinson, G.; Gregson, W. Factors influencing perception of effort (session rating of perceived exertion) during elite soccer training. Int. J. Sports Physiol. Perform. 2015, 10, 860-864. [CrossRef]

119. Cunniffe, B.; Proctor, W.; Baker, J.S.; Davies, B. An evaluation of the physiological demands of elite rugby union using global positioning system tracking software. J. Strength Cond. Res. 2009, 23, 1195-1203. [CrossRef] [PubMed]

120. Di Prampero, P.E.; Fusi, S.; Sepulcri, L.; Morin, J.B.; Belli, A.; Antonutto, G. Sprint running: A new energetic approach. J. Exp. Biol. 2005, 208, 2809-2816. [CrossRef] [PubMed]

121. Lago, C. The influence of match location, quality of opposition, and match status on possession strategies in professional association football. J. Sports Sci. 2009, 27, 1463-1469. [CrossRef]

122. Lago, C.; Casais, L.; Dominguez, E.; Sampaio, J. The effects of situational variables on distance covered at various speeds in elite soccer. Eur. J. Sport Sci. 2010, 10, 103-109. [CrossRef]

123. Carling, C.; Gregson, W.; McCall, A.; Moreira, A.; Wong, D.P.; Bradley, P.S. Match running performance during fixture congestion in elite soccer: Research issues and future directions. Sports Med. 2015, 45, 605-613. [CrossRef]

124. Malone, J.J.; Jaspers, A.; Helsen, W.; Merks, B.; Frencken, W.G.; Brink, M.S. Seasonal training load and wellness monitoring in a professional soccer goalkeeper. Int. J. Sports Physiol. Perform. 2018, 13, 672-675. [CrossRef]

125. Johnston, R.J.; Watsford, M.L.; Kelly, S.J.; Pine, M.J.; Spurrs, R.W. Validity and interunit reliability of $10 \mathrm{~Hz}$ and $15 \mathrm{~Hz}$ GPS units for assessing athlete movement demands. J. Strength Cond. Res. 2014, 28, 1649-1655. [CrossRef]

126. Scott, M.T.; Scott, T.J.; Kelly, V.G. The validity and reliability of global positioning systems in team sport: A brief review. J. Strength Cond. Res. 2016, 30, 1470-1490. [CrossRef] [PubMed]

127. Kempton, T.; Sullivan, C.; Bilsborough, J.C.; Cordy, J.; Coutts, A.J. Match-to-match variation in physical activity and technical skill measures in professional Australian Football. J. Sci. Med. Sport 2015, 18, 109-113. [CrossRef] [PubMed]

128. Peeters, A.; Carling, C.; Piscione, J.; Lacome, M. In-Match physical performance fluctuations in international rugby sevens competition. J. Sports Sci. Med. 2019, 18, 419-426. [PubMed]

129. Sweeting, A.J.; Aughey, R.J.; Cormack, S.J.; Morgan, S. Discovering frequently recurring movement sequences in team-sport athlete spatiotemporal data. J. Sports Sci. 2017, 35, 2439-2445. [CrossRef]

130. Lloyd, R.S.; Oliver, J.L. The youth physical development model: A new approach to long-term athletic development. Strength Cond. J. 2012, 34, 61-72. [CrossRef]

131. Harper, D.J.; Carling, C.; Kiely, J. High-intensity acceleration and deceleration demands in elite team sports competitive match play: A systematic review and meta-analysis of observational studies. Sports Med. 2019, 49, 1923-1947. [CrossRef] [PubMed] 